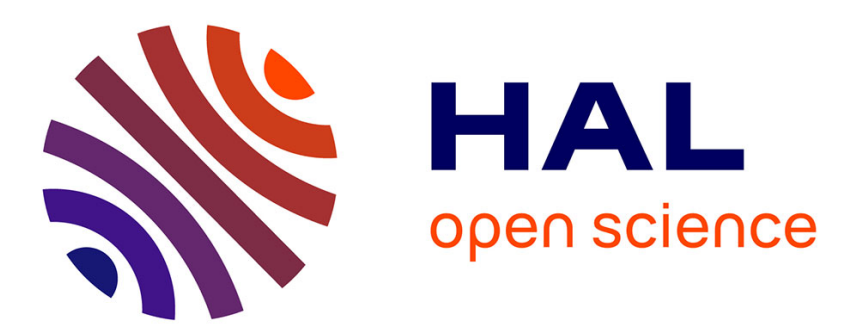

\title{
Coupling schemes for incompressible fluid-structure interaction: implicit, semi-implicit and explicit
}

\author{
Miguel Angel Fernández
}

\section{To cite this version:}

Miguel Angel Fernández. Coupling schemes for incompressible fluid-structure interaction: implicit, semi-implicit and explicit. SeMA Journal: Boletin de la Sociedad Española de Matemática Aplicada, 2011, 55, pp.59-108. 10.1007/BF03322593 . inria-00580772v2

\section{HAL Id: inria-00580772 https://hal.inria.fr/inria-00580772v2}

Submitted on 5 Apr 2011

HAL is a multi-disciplinary open access archive for the deposit and dissemination of scientific research documents, whether they are published or not. The documents may come from teaching and research institutions in France or abroad, or from public or private research centers.
L'archive ouverte pluridisciplinaire HAL, est destinée au dépôt et à la diffusion de documents scientifiques de niveau recherche, publiés ou non, émanant des établissements d'enseignement et de recherche français ou étrangers, des laboratoires publics ou privés. 


\title{
Coupling schemes for incompressible fluid-structure interaction: implicit, semi-implicit and explicit
}

\author{
Miguel A. Fernández \\ INRIA, Rocquencourt, BP 105 \\ F-78153 Le Chesnay Cedex, France \\ miguel.fernandez@inria.fr
}

\begin{abstract}
Over the last decade, the numerical simulation of incompressible fluidstructure interaction has been a very active research field and the subject of numerous works. This is due, in particular, to the increasing interest of the research community in the simulation of blood flows in large arteries. In this context, the fluid equations have to be solved in a moving domain and the incompressibility constraint makes the coupling sensitive to the added-mass effect. As a result, the solution procedure has to be designed carefully in order to guarantee efficiency without compromising numerical stability. In this paper, we review some of the coupling schemes recently proposed in the literature. Some numerical results that show the effectiveness of the novel approaches are also presented.
\end{abstract}

Key words : Fluid-structure interaction, incompressible flow, NavierStokes equation, elastodynamics, coupling schemes, partitioned procedures, numerical stability.

AMS subject classifications : $\quad 74 F 10,65 M 12$ 


\section{Contents}

1. Introduction 3

2. Preliminaries 5

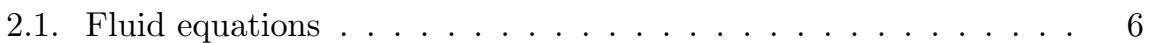

2.2. Solid equations . . . . . . . . . . . . . . 7

2.3. Interface coupling conditions . . . . . . . . . . 7

2.4. Summary of the equations and global energy balance . . . . . 8

3. Implicit coupling 10

3.1. Variational setting . . . . . . . . . . . . . . . . . 11

3.2. Partitioned solution methods . . . . . . . . . . . . . . . 14

4. Semi-implicit coupling 19

4.1. Projection-based semi-implicit coupling . . . . . . . . . . 19

5. Nitsche's based interface treatment 26

5.1. Stabilized explicit coupling . . . . . . . . . . . 26

5.2. Robin-based semi-implicit coupling . . . . . . . . . . . 36

6. Conclusion 41 


\section{Introduction}

Computational Fluid-Structure Dynamics (CFSD) is of great importance in practically all engineering fields, from aeroelasticity to biomechanics (see, e.g., $[40,93,99,85,92,113,59,118,14,70,28,42,114,8,120,33])$. The work summarized in this review stems from the numerical simulation of the (mechanical) interaction between blood flow and the vessel wall in large arteries. Over the last decade, this topic has been a very active field of research and the subject of numerous works (see, e.g., [96, 59, 118, 87, 51, 121, 76, 77, 53]). The underlying motivation is that computer based simulations of blood flows, in patient-specific geometries, can provide valuable information to physicians (e.g., in order to enhance diagnosis and therapy planing). Moreover, such simulations can also be a major ingredient in the design/optimization of medical devices.

The numerical simulation of the fluid-structure phenomena involved in blood flows raises many issues. Among them, the displacement of the wall cannot be supposed to be infinitesimal, geometrical nonlinearities are therefore present in the structure and the fluid has to be solved in a moving domain. On the other hand, since blood is an incompressible fluid, the arteries are slender and the vessel and blood densities close, the coupling has to be tackled carefully in order to avoid numerical instabilities.

In large (or medium size) arteries, blood is commonly modeled as a homogeneous, viscous, Newtonian and incompressible fluid (see, e.g., $[115,53]$ ). Although the artery wall has a viscoelastic behavior (see, e.g., [56]), we limit the presentation to the case of a non-linear elastic solid. Yet, the coupling strategies discussed below are not restricted to this structural behavior. As a mathematical model, we consider therefore the system of partial differential equations involving the Navier-Stokes equation (in a moving domain), the non-

linear elastodynamics equation and the following coupling conditions on the interface $\Sigma$ :

- continuity of displacements (fluid domain and structure):

$$
\boldsymbol{d}^{\mathrm{f}}=\boldsymbol{d}^{\mathrm{s}} \quad \text { on } \quad \Sigma ;
$$

- continuity of velocity (fluid and structure):

$$
\boldsymbol{u}^{\mathrm{f}}=\boldsymbol{u}^{\mathrm{s}} \quad \text { on } \quad \Sigma \text {; }
$$

- equilibrium of stresses (structure and fluid):

$$
\boldsymbol{\sigma}^{\mathrm{s}} \boldsymbol{n}^{\mathrm{s}}=-\boldsymbol{\sigma}^{\mathrm{f}} \boldsymbol{n}^{\mathrm{f}} \quad \text { on } \quad \Sigma .
$$

This paper concerns the numerical resolution of this coupled problem.

The time semi-discretizations of this system exploit, in general, the heterogeneous structure of the coupled problem. That is, the fluid and the solid are time semi-discretized by different time-marching schemes, tailored by 
their different mathematical properties. On the other hand, the time semidiscretization of the interface coupling conditions (1)-(3) defines the coupling scheme.

One of the most elementary coupling schemes (perhaps the most popular in the aeroelastic community) is based on the following explicit treatment of (1) and (2):

$$
\left\{\begin{array}{rcc}
\boldsymbol{d}^{\mathrm{f}, n+1}=\boldsymbol{d}^{\mathrm{s}, n} & \text { on } & \Sigma, \\
\boldsymbol{u}^{\mathrm{f}, n+1}=\boldsymbol{u}^{\mathrm{s}, n} & \text { on } & \Sigma, \\
\boldsymbol{\sigma}^{\mathrm{s}, n+1} \boldsymbol{n}^{\mathrm{s}}=\boldsymbol{\sigma}^{\mathrm{f}, n+1} \boldsymbol{n}^{\mathrm{f}} & \text { on } & \Sigma .
\end{array}\right.
$$

This yields the procedure reported in Algorithm 1. This algorithm is known as conventional serial staggered scheme (see, e.g., $[100,86,102,42])$. Note that Algorithm 1 is very appealing in terms of computational cost, since it allows a fully uncoupled (sequential) solution of the discrete problem.

Algorithm 1 Explicit coupling (weakly or loosely coupled) scheme.

1. Update the fluid domain configuration (mesh) and velocities via (4)

2. Advance in time the fluid with the interface Dirichlet condition $(4)_{2}$;

3. Advance in time the structure with the interface Neumann condition $(4)_{3}$;

4. Go to next time-step.

Explicit coupling (weakly or loosely coupled) schemes are those in which (2) or (3) are explicitly treated. A spurious numerical power is therefore generated at the interface (energy is not exactly balanced due to the explicit treatment), which has to be controlled in order to guarantee stability. Algorithm 1 is an explicit coupling scheme, since it treats (2) explicitly. Although explicit coupling algorithms are widely and successfully used in aeroelasticity (see, e.g., $[101,102,55,42]$ ), a number of numerical studies (see, e.g., $[91,85,105,96,59]$ ) have shown that Algorithm 1 is unstable under certain choices of the physical parameters. Typically, this happens when the fluid is incompressible, the fluid and solid densities are comparable or when the domain has a slender shape, irrespectively of the choice of the time-step size. Blood flows are a popular example of such a situation.

Theoretical explanations of this issue have been reported in [25] (see also [54]). In particular, the following instability condition is established in [25] for a simplified framework:

$$
\frac{\rho^{\mathrm{S}} \epsilon}{\rho^{\mathrm{f}} \lambda_{\text {add }}}<1,
$$

where $\epsilon$ and $\lambda_{\text {add }}$ are pure geometrical quantities. The former is related to the thickness of the structure, whereas the latter increases with the length of the domain (it is the largest eigenvalue of the so-called added-mass interface operator). Note that the left hand-side of (5) is a pure physical quantity, it 
measures the amount of added-mass effect in the system. In particular, since (5) is independent of the time-step size, reducing it does not cure the instabilities (as mentioned above).

Implicit coupling schemes are those that time semi-discretize (1)-(3) implicitly. The schemes that treat (2) and (3) in an implicit fashion are also known as strongly coupled. The implicit coupling schemes are therefore strongly coupled. These schemes have been, for years, the unique way of circumventing the above mentioned numerical instabilities. Somehow, this explains why the development of efficient methods for the resolution of the coupled non-linear systems, arising in implicit coupling, has been (and still is) a very active field of research.

Some of these implicit coupling procedures are described in $\S 3$. In particular, we present the Newton algorithm proposed in [49]. The rest of the paper is devoted to the problem of avoiding strong coupling, without compromising stability. This issue is addressed from two different perspectives. In $\S 4$, we present the semi-implicit coupling paradigm proposed in [48]. A different point of view is considered in $\S 5$, where we review the schemes recently proposed in $[22,5]$, that are based on the weak treatment of the interface conditions at the (space) discrete level. Some final remarks and lines of future work are drawn in $\S 6$.

The next section contains introductory material. We review there the main ingredients of the general mathematical model used to describe the interaction of a viscous incompressible Newtonian fluid and an elastic structure.

\section{Preliminaries}

The modeling of fluid-structure interaction systems under large displacements involves, in a general way, the coupling of two formulations: the solid classically treated in Lagrangian formulation, and the fluid described by an arbitrary Lagrangian-Eulerian (ALE) formulation (see, e.g., [40, 98, 90, 85, 45]).

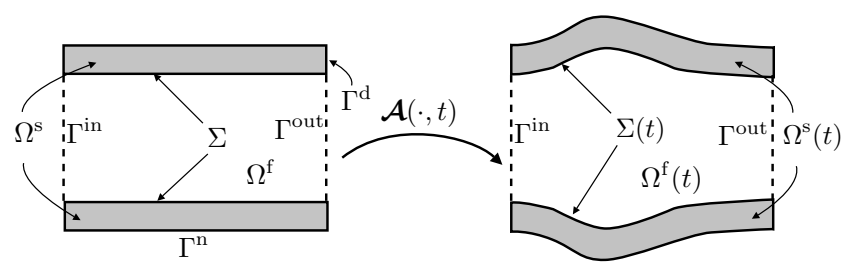

Figure 1: Geometrical fluid-structure configurations.

We consider a mechanical system occupying a moving domain which consists of a deformable structure $\Omega^{\mathrm{s}}(t)$ (e.g., the vessel wall) interacting with a fluid under motion (e.g, the blood) in the complement $\Omega^{\mathrm{f}}(t)$ of $\Omega^{\mathrm{s}}(t)$, see Figure 1 . We denote by $\Sigma(t)$ the current configuration of the fluid-structure interface, that is, $\Sigma(t) \stackrel{\text { def }}{=} \partial \Omega^{\mathrm{f}}(t) \cap \partial \Omega^{\mathrm{s}}(t)$. Let $\Omega^{\mathrm{f}} \cup \Omega^{\mathrm{s}}$ be a reference configuration of 
the system (e.g., the initial configuration). We denote by $\Sigma \stackrel{\text { def }}{=} \partial \Omega^{\mathrm{f}} \cap \partial \Omega^{\mathrm{s}}$ the reference fluid-solid interface and $\partial \Omega^{\mathrm{f}}=\Gamma^{\text {in }} \cup \Gamma^{\text {out }} \cup \Sigma, \partial \Omega^{\mathrm{s}}=\Gamma^{\mathrm{d}} \cup \Gamma^{\mathrm{n}} \cup \Sigma$, are given partitions of the fluid and solid boundaries respectively. The fluid external boundaries $\Gamma^{\text {in }}$ and $\Gamma^{\text {out }}$ are supposed to be fixed. The corresponding outward normal vectors to the fluid and solid boundaries are denoted by $\boldsymbol{n}$ and $\boldsymbol{n}^{\mathrm{s}}$, respectively (the same notation is used for their reference and current configurations).

\subsection{Fluid equations}

The dynamics of the (moving) control volume $\Omega^{\mathrm{f}}(t)$ are parametrized in terms of a smooth injective map $\mathcal{A}: \Omega^{\mathrm{f}} \times \mathbb{R}^{+} \rightarrow \mathbb{R}^{d}$, the so-called ALE-map, such that

$$
\Omega^{\mathrm{f}}(t)=\mathcal{A}_{t}\left(\Omega^{\mathrm{f}}\right),
$$

with the notation $\mathcal{A}_{t} \stackrel{\text { def }}{=} \mathcal{A}(\cdot, t)$. The corresponding deformation gradient and Jacobian are denoted by $\boldsymbol{F} \stackrel{\text { def }}{=} \boldsymbol{\nabla} \mathcal{A}_{t}$ and $J \stackrel{\text { def }}{=} \operatorname{det} \boldsymbol{F}$, respectively. Moreover, we shall use the notation $\boldsymbol{w} \stackrel{\text { def }}{=} \partial_{t} \mathcal{A}$ for the fluid domain velocity, and $\boldsymbol{d}^{\mathrm{f}}(\widehat{\boldsymbol{x}}, t) \stackrel{\text { def }}{=} \mathcal{A}_{t}(\widehat{\boldsymbol{x}})-\widehat{\boldsymbol{x}}, \widehat{\boldsymbol{x}} \in \Omega^{\mathrm{f}}$, for the fluid domain displacement.

Remark 1 Thanks to the invertibility of $\mathcal{A}_{t}$, we can define all the physical quantities on the reference or on the current configuration, the choice being a matter of convenience. When the same field is evaluated in both the current and the reference configurations, we adopt the superscript ${ }^{\wedge}{ }^{~}$ to indicate that it is defined in $\Omega^{\mathrm{f}} \times \mathbb{R}^{+}$and we have the relations

$$
\begin{aligned}
& \widehat{q}(\widehat{\boldsymbol{x}}, t) \stackrel{\text { def }}{=} q\left(\mathcal{A}_{t}(\widehat{\boldsymbol{x}}), t\right) \quad \forall \widehat{\boldsymbol{x}} \in \Omega^{\mathrm{f}}, \\
& q(\boldsymbol{x}, t) \stackrel{\text { def }}{=} \widehat{q}\left(\mathcal{A}_{t}^{-1}(\boldsymbol{x}), t\right) \quad \forall \boldsymbol{x} \in \Omega^{\mathrm{f}}(t) .
\end{aligned}
$$

In the rest of situations (i.e., a field is only used in one of the configurations), the superscript ${ }^{-}$is not used.

We assume the fluid to be homogeneous, Newtonian and incompressible. Its behavior is described in terms of its velocity $\widehat{\boldsymbol{u}}: \Omega^{\mathrm{f}} \times \mathbb{R}^{+} \rightarrow \mathbb{R}^{d}$ and pressure $\widehat{p}: \Omega^{\mathrm{f}} \times \mathbb{R}^{+} \rightarrow \mathbb{R}$ fields, which are governed by the following Navier-Stokes equations (written in ALE form):

$$
\left\{\begin{aligned}
\left.\rho^{\mathrm{f}} \partial_{t} \boldsymbol{u}\right|_{\mathcal{A}}+\rho^{\mathrm{f}}(\boldsymbol{u}-\boldsymbol{w}) \cdot \boldsymbol{\nabla} \boldsymbol{u}-\operatorname{div} \boldsymbol{\sigma}(\boldsymbol{u}, p)=\mathbf{0} & \text { in } \quad \Omega^{\mathrm{f}}(t), \\
\operatorname{div} \boldsymbol{u}=0 & \text { in } \quad \Omega^{\mathrm{f}}(t),
\end{aligned}\right.
$$

where $\rho^{\mathrm{f}}$ stands for the fluid density, $\left.\partial_{t} \boldsymbol{u}\right|_{\mathcal{A}}$ for the ALE time derivative and $\boldsymbol{\sigma}(\boldsymbol{u}, p) \stackrel{\text { def }}{=}-p \boldsymbol{I}+2 \mu \boldsymbol{\epsilon}(\boldsymbol{u})$ for the fluid Cauchy stress tensor, with $\mu$ the fluid dynamic viscosity and $\boldsymbol{\epsilon}(\boldsymbol{u}) \stackrel{\text { def }}{=} \frac{1}{2}\left(\boldsymbol{\nabla} \boldsymbol{u}+\boldsymbol{\nabla} \boldsymbol{u}^{\mathrm{T}}\right)$ the strain rate tensor.

System (6) has to be supplemented with boundary conditions, for instance,

$$
\left\{\begin{array}{rc}
\boldsymbol{u}=\boldsymbol{u}_{\text {in }} & \text { on } \quad \Gamma^{\text {in }}, \\
\boldsymbol{\sigma}(\boldsymbol{u}, p) \boldsymbol{n}=-p_{\text {out }} \boldsymbol{n} & \text { on } \quad \Gamma^{\text {out }},
\end{array}\right.
$$


and initial condition $\left.\boldsymbol{u}\right|_{t=0}=\boldsymbol{u}_{0}$. Here, $\boldsymbol{u}_{\text {in }}, p_{\text {out }}$ and $\boldsymbol{u}_{0}$ are given boundary and initial data. The conditions to be enforced on the fluid-structure interface $\Sigma(t)$ are discussed in $\S 2.3$.

Remark 2 Note that the importance of the presence of the ALE time-derivative $\left.\partial_{t} \boldsymbol{u}\right|_{\mathcal{A}}$ in (6) emerges in the context of the numerical discretisation (we recall that $\left.\widehat{\left.\partial_{t} \boldsymbol{u}\right|_{\mathcal{A}}} \stackrel{\text { def }}{=} \partial_{t} \widehat{\boldsymbol{u}}\right)$. Indeed, when computing numerically a solution in a moving domain we are usually interested in the time variation of quantities collocated at the nodes of a computational mesh (not at a particular fixed position), and the latter necessarily follows the evolution of the computational domain.

\subsection{Solid equations}

The dynamics of the structure are parametrized in terms of its displacement $\boldsymbol{d}: \Omega^{\mathrm{s}} \times \mathbb{R}^{+} \rightarrow \mathbb{R}^{d}$. Its evolution is generally governed by the non-linear elastodynamics equations

$$
\left\{\begin{array}{rll}
\rho^{\mathrm{s}} \partial_{t} \dot{\boldsymbol{d}}-\operatorname{div}(\boldsymbol{\Pi}(\boldsymbol{d}))=\mathbf{0} & \text { in } & \Omega^{\mathrm{s}}, \\
\dot{\boldsymbol{d}}=\partial_{t} \boldsymbol{d} & \text { in } & \Omega^{\mathrm{s}},
\end{array}\right.
$$

where $\rho^{\mathrm{s}}$ represents the solid density, $\dot{\boldsymbol{d}}$ the velocity and $\boldsymbol{\Pi}(\boldsymbol{d})$ the first PiolaKirchhoff stress tensor of the structure. The latter being related to $\boldsymbol{d}$ through an appropriate constitutive law (see, e.g., $[66,30,82]$ ). For instance, for an hyper-elastic material, we have

$$
\boldsymbol{\Pi}(\boldsymbol{d})=\boldsymbol{F}^{\mathrm{s}} \frac{\partial W}{\partial \boldsymbol{E}}\left(\boldsymbol{E}^{\mathrm{s}}\right),
$$

where $\boldsymbol{F}^{\mathrm{s}} \stackrel{\text { def }}{=} \boldsymbol{I}+\boldsymbol{\nabla} \boldsymbol{d}_{t}$ stands for the gradient of deformation, $\boldsymbol{E}^{\mathrm{s}} \stackrel{\text { def }}{=} \frac{1}{2}\left(\left(\boldsymbol{F}^{\mathrm{s}}\right)^{\mathrm{T}} \boldsymbol{F}^{\mathrm{s}}-\right.$ I) for the Green-Lagrange strain tensor and $W: \mathbb{R}^{d \times d} \rightarrow \mathbb{R}^{+}$is a given density of elastic energy.

The solid equation (7) has to be supplemented also with boundary conditions, for instance,

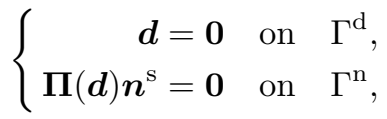

and initial conditions $\left.\boldsymbol{d}\right|_{t=0}=\boldsymbol{d}^{0},\left.\partial_{t} \boldsymbol{d}\right|_{t=0}=\dot{\boldsymbol{d}}^{0}$. The boundary conditions to be enforced on $\Sigma$ are discussed in the next subsection.

\subsection{Interface coupling conditions}

In order to ensure a correct energy balance, both the kinematic and the kinetic continuity need to be enforced across the fluid-structure interface at all times (see, e.g., [85] and [45]). The equilibrium of stresses is given (in the reference configuration) by

$$
\boldsymbol{\Pi}(\boldsymbol{d}) \boldsymbol{n}^{\mathrm{s}}=-J \widehat{\boldsymbol{\sigma}}(\boldsymbol{u}, p) \boldsymbol{F}^{-\mathrm{T}} \boldsymbol{n} \quad \text { on } \quad \Sigma .
$$


The continuity of the velocity is enforced by setting

$$
\widehat{\boldsymbol{u}}=\partial_{t} \boldsymbol{d} \quad \text { on } \quad \Sigma .
$$

The fluid domain displacement is taken such that

$$
\boldsymbol{d}^{\mathrm{f}}=\boldsymbol{d} \quad \text { on } \quad \Sigma,
$$

that is, the fluid and solid domains remain sticked at all times. This last equality with (8) yields $\boldsymbol{u}=\boldsymbol{w}$ on $\Sigma(t)$.

Note that, since we have assumed (for simplicity) that the inlet and outlet boundaries $\left(\Gamma^{\text {in }}, \Gamma^{\text {out }}\right)$ remain fixed, we have

$$
\boldsymbol{d}^{\mathrm{f}}=\mathbf{0} \quad \text { on } \quad \Gamma^{\text {in }} \cup \Gamma^{\text {out }} .
$$

Therefore equations (2.3) and (9) constrain the value of $\boldsymbol{d}^{\mathrm{f}}$ on the whole boundary $\partial \Omega^{\mathrm{f}}$. Inside $\Omega^{\mathrm{f}}$, however, the displacement $\boldsymbol{d}^{\mathrm{f}}$ (and hence the map $\mathcal{A}$ ) is arbitrary: it can be any reasonable extension of $\left.\boldsymbol{d}\right|_{\Sigma}$ over $\Omega^{\mathrm{f}}$ (subjected to (9)). In the sequel we will denote this operation by

$$
\boldsymbol{d}^{\mathrm{f}}=\operatorname{Ext}\left(\left.\boldsymbol{d}\right|_{\Sigma}\right)
$$

For instance, the operator Ext can be given in terms of an harmonic extension, by solving:

$$
\left\{\begin{aligned}
-\boldsymbol{\Delta} \boldsymbol{d}^{\mathrm{f}}=\mathbf{0} & \text { in } \quad \Omega^{\mathrm{f}} \\
\boldsymbol{d}^{\mathrm{f}}=\mathbf{0} & \text { on } \quad \Gamma^{\mathrm{in}} \cup \Gamma^{\mathrm{out}}, \\
\boldsymbol{d}^{\mathrm{f}}=\boldsymbol{d} & \text { on } \quad \Sigma .
\end{aligned}\right.
$$

In summary, the interface coupling conditions are given by:

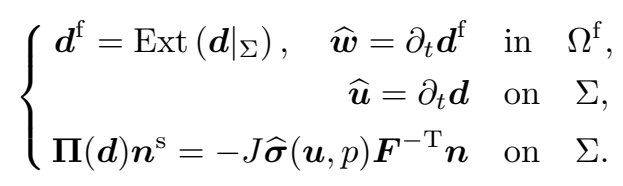

Remark 3 The main ingredients of the ALE (arbitrary Lagrangian-Eulerian) formalism can be inferred from (10). Indeed, the conditions $(10)_{1,2}$ impose that the interface points must follow the same displacement as the fluid, thus the Lagrangian terminology. In contrast, the motion of the remaining points is not necessarily related to the fluid kinematics, so the Eulerian terminology.

\subsection{Summary of the equations and global energy balance}

As mathematical model, we consider therefore the system of partial differential equations involving the Navier-Stokes equations (6), the non-linear elastodynamics equations (7) and the interface coupling conditions (10). This yields the following problem: find the fluid domain displacement $\boldsymbol{d}^{\mathrm{f}}: \Omega^{\mathrm{f}} \times \mathbb{R}^{+} \rightarrow$ 
$\mathbb{R}^{d}$, the fluid velocity $\widehat{\boldsymbol{u}}: \Omega^{\mathrm{f}} \times \mathbb{R}^{+} \rightarrow \mathbb{R}^{d}$, the fluid pressure $\widehat{p}: \Omega^{\mathrm{f}} \times \mathbb{R}^{+} \rightarrow \mathbb{R}$ and the structure displacement $\boldsymbol{d}: \Omega^{\mathrm{s}} \times \mathbb{R}^{+} \rightarrow \mathbb{R}^{d}$ such that

$$
\begin{aligned}
& \left\{\begin{array}{rccc}
\left.\rho^{\mathrm{f}} \partial_{t} \boldsymbol{u}\right|_{\mathcal{A}}+\rho^{\mathrm{f}}(\boldsymbol{u}-\boldsymbol{w}) \cdot \boldsymbol{\nabla} \boldsymbol{u}-\operatorname{div} \boldsymbol{\sigma}(\boldsymbol{u}, p)=\mathbf{0} & \text { in } & \Omega^{\mathrm{f}}(t), \\
\operatorname{div} \boldsymbol{u}=0 & \text { in } & \Omega^{\mathrm{f}}(t), \\
\boldsymbol{u}=\boldsymbol{u}_{\text {in }} & \text { on } & \Gamma^{\text {in }}, \\
\boldsymbol{\sigma}(\boldsymbol{u}, p) \boldsymbol{n}=-p_{\text {out }} \boldsymbol{n} & \text { on } & \Gamma^{\text {out }},
\end{array}\right. \\
& \left\{\begin{aligned}
\rho^{\mathrm{s}} \partial_{t} \dot{\boldsymbol{d}}-\operatorname{div}(\boldsymbol{\Pi}(\boldsymbol{d}))=\mathbf{0} & \text { in } \quad \Omega^{\mathrm{s}}, \\
\dot{\boldsymbol{d}}=\partial_{t} \boldsymbol{d} & \text { in } \quad \Omega^{\mathrm{s}}, \\
\boldsymbol{d}=\mathbf{0} & \text { on } \quad \Gamma^{\mathrm{d}}, \\
\boldsymbol{\Pi}(\boldsymbol{d}) \boldsymbol{n}^{\mathrm{s}}=\mathbf{0} & \text { on } \quad \Gamma^{\mathrm{n}},
\end{aligned}\right.
\end{aligned}
$$

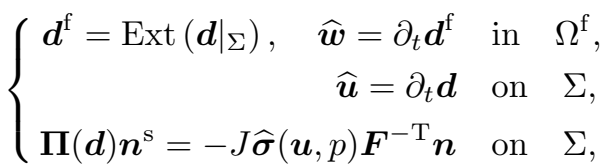

with the initial conditions $\left.\boldsymbol{u}\right|_{t=0}=\boldsymbol{u}^{0},\left.\boldsymbol{d}\right|_{t=0}=\boldsymbol{d}^{0}$ and $\left.\partial_{t} \boldsymbol{d}\right|_{t=0}=\dot{\boldsymbol{d}}^{0}$. We refer to [88] for a recent review on the mathematical analysis of this type of coupled problems. This review paper is devoted to the numerical resolution of (11)-(13).

The next result (see, e.g., [94] and [46] for a proof) shows that the coupled system (11)-(13) ensures a correct balance of the mechanical energy.

Lemma 1 Assume that the structure is hyper-elastic (with energy density function $W$ ) and that the coupled fluid-structure system is isolated, i.e., $\boldsymbol{u}=\mathbf{0}$ on $\Gamma^{\text {in }} \cup \Gamma^{\text {out }}$. Let

$$
E(t) \stackrel{\text { def }}{=} \underbrace{\int_{\Omega^{\mathrm{f}}(t)} \frac{\rho^{\mathrm{f}}}{2}|\boldsymbol{u}|^{2}+\int_{\Omega^{\mathrm{s}}} \frac{\rho^{\mathrm{s}}}{2}|\dot{\boldsymbol{d}}|^{2}}_{\begin{array}{c}
\text { Kinetic } \\
\text { energy }
\end{array}}+\underbrace{\int_{\Omega^{\mathrm{s}}} W\left(\boldsymbol{E}^{\mathrm{s}}(\boldsymbol{d})\right)}_{\begin{array}{c}
\text { Elastic } \\
\text { potential energy }
\end{array}}
$$

be the total mechanical energy of the fluid-structure system described by (11)(13). Then, the following energy identity holds:

$$
E(t)=E(0)-\underbrace{\int_{0}^{t} \int_{\Omega^{\mathrm{f}}(t)} 2 \mu|\boldsymbol{\epsilon}(\boldsymbol{u})|^{2}}_{\text {Viscous work }} .
$$

As expected, dissipation only comes from the fluid viscous effects and the power exchanged by the fluid and the structure exactly balance at the interface. This balance is a direct consequence of the interface coupling conditions (13). 


\section{Implicit coupling}

In what follows, $\tau>0$ denotes a given time-step size and $x^{n}$ an approximation of a given time-dependent field $x$ at time $t_{n} \stackrel{\text { def }}{=} n \tau$, with $n \in \mathbb{N}$. Moreover, $\partial_{\tau} x^{n+1} \stackrel{\text { def }}{=}\left(x^{n+1}-x^{n}\right) / \tau$ denotes the first order backward difference and $x^{n+\frac{1}{2}} \stackrel{\text { def }}{=}\left(x^{n+1}+x^{n}\right) / 2$ the mid-point value approximation.

The time semi-discretizations of (11)-(13) exploit, in general, the heterogeneous structure of the coupled problem. That is, (11) and (12) are time semi-discretized by different time-marching schemes, tailored by their different mathematical properties. To fix the ideas, we consider an implicit scheme for the ALE Navier-Stokes equations (11),

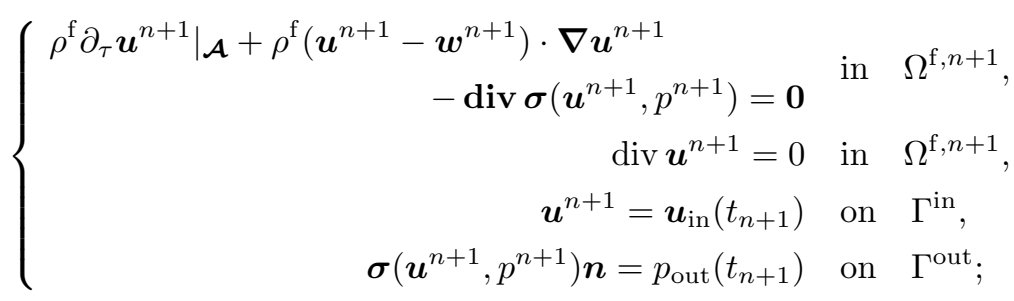

and a mid-point rule for the structural equation (12),

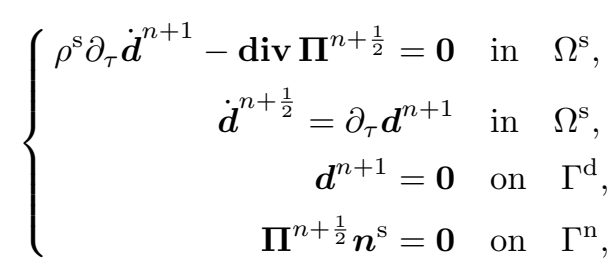

with the notation $\boldsymbol{\Pi}^{n+\frac{1}{2}} \stackrel{\text { def }}{=} \frac{1}{2}\left(\boldsymbol{\Pi}\left(\boldsymbol{d}^{n+1}\right)+\boldsymbol{\Pi}\left(\boldsymbol{d}^{n}\right)\right)$.

As mentioned in the previous section, explicit coupling schemes may lead to numerical instabilities. These numerical instabilities have been traditionally circumvented by considering fully implicit time-discretizations of (11)-(13). For instance, by combining (14) and (15) with the following implicit treatment of (13):

$$
\left\{\begin{array}{l}
\boldsymbol{d}^{\mathrm{f}, n+1}=\operatorname{Ext}\left(\left.\boldsymbol{d}^{n+1}\right|_{\Sigma}\right), \quad \widehat{\boldsymbol{w}}^{n+1}=\partial_{\tau} \boldsymbol{d}^{\mathrm{f}, n+1}, \quad \Omega^{\mathrm{f}, n+1}=\left(\boldsymbol{I}_{\Omega^{\mathrm{f}}}+\boldsymbol{d}^{\mathrm{f}, n+1}\right)\left(\Omega^{\mathrm{f}}\right), \\
\widehat{\boldsymbol{u}}^{n+1}=\partial_{\tau} \boldsymbol{d}^{n+1} \quad \text { on } \quad \Sigma . \\
\boldsymbol{\Pi}^{n+\frac{1}{2}} \boldsymbol{n}^{\mathrm{s}}=-J^{n+1} \widehat{\boldsymbol{\sigma}}\left(\boldsymbol{u}^{n+1}, p^{n+1}\right)\left(\boldsymbol{F}^{n+1}\right)^{-\mathrm{T}} \boldsymbol{n} \quad \text { on } \quad \Sigma .
\end{array}\right.
$$

This yields the time-marching procedure summarized in Algorithm 2.

Note that Algorithm 2 is an implicit coupling (so, strongly coupled) scheme, since (13) is enforced exactly at each time-step. As a result, the scheme can be proved to satisfy a discrete counterpart of Lemma 1 and, therefore, is energy stable (under a $G C L$ condition, see [94, 85, 83] and [46]). The payoff of this enhanced stability is that the equations (14), (15) and (16) yield a 


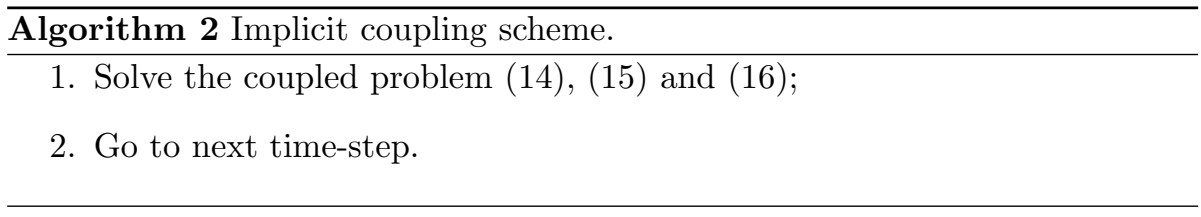

highly nonlinear coupled system at each time-step. As a matter of fact, in addition to the common nonlinearities of the fluid and solid equations, implicit coupling induces geometrical nonlinearities within the fluid equations, due to the dependence of $\Omega^{\mathrm{f}, \mathrm{n}+1}$ on $\boldsymbol{d}^{\mathrm{f}, n+1}$.

The solution procedures for this coupled non-linear problem (and for coupled problems, in general) are commonly classified into two distinct categories: monolithic and partitioned (see, e.g., [43]). An ad hoc single solver whose purpose is to simultaneously solve (14)-(15) and (16) leads to a monolithic procedure (see, e.g., $[105,113,70,14,72,51,15,11,77,58])$ ). A solution method that couples independent fluid (14) and structure (15) solvers is termed a partitioned procedure (see, e.g., [104, 92, 85, 89, 59, 38, 80, 36, 78, 9, 73, 32, 33]).

Remark 4 Needless to say that Algorithm 1 is a partitioned procedure.

Monolithic methods are, by construction, less modular than partitioned approaches and do not allow the use of legacy software. Partitioned methods, on the contrary, facilitate the reuse of existing code. Moreover, because of their inherent modularity, new models and numerical schemes can be introduced while keeping everything else the same (see, e.g., $[117,39,6]$ ). All these advantages come, however, with a price: computational efficiency over a monolithic approach is not necessarily guaranteed (see [71, 11, 77]).

\subsection{Variational setting}

In variational form, the non-linear coupled system (14), (15) and (16) can be formulated as the following monolithic problem (see, e.g., [46]): for $n \geq 0$, find $\boldsymbol{d}^{\mathrm{f}, n+1} \in \boldsymbol{H}_{\Gamma^{\text {in }} \cup \Gamma^{\text {out }}}^{1}\left(\Omega^{\mathrm{f}}\right), \widehat{\boldsymbol{u}}^{n+1} \in \boldsymbol{H}^{1}\left(\Omega^{\mathrm{f}}\right), \widehat{p}^{n+1} \in L^{2}\left(\Omega^{\mathrm{f}}\right)$ and $\boldsymbol{d}^{n+1} \in \overline{\boldsymbol{H}}_{\Gamma^{\mathrm{d}}}^{1}\left(\Omega^{\mathrm{d}}\right)$ with $\left.\boldsymbol{u}^{n+1}\right|_{\Gamma^{\text {in }}}=\boldsymbol{u}_{\text {in }}\left(t_{n+1}\right)$, satisfying $(16)_{1,2}$ and such that

$$
\begin{array}{r}
\frac{\rho^{\mathrm{f}}}{\tau}\left(\int_{\Omega^{\mathrm{f}, n+1}} \boldsymbol{u}^{n+1} \cdot \boldsymbol{v}^{\mathrm{f}}-\int_{\Omega^{\mathrm{f}, n}} \boldsymbol{u}^{n} \cdot \boldsymbol{v}^{\mathrm{f}}\right)+\rho^{\mathrm{f}} \int_{\Omega^{\mathrm{f}, n+1}}\left(\boldsymbol{u}^{n+1}-\boldsymbol{w}^{n+1}\right) \cdot \boldsymbol{\nabla} \boldsymbol{u}^{n+1} \cdot \boldsymbol{v}^{\mathrm{f}} \\
-\rho^{\mathrm{f}} \int_{\Omega^{\mathrm{f}, n+1}}\left(\operatorname{div} \boldsymbol{w}^{n+1}\right) \boldsymbol{u} \cdot \boldsymbol{v}^{\mathrm{f}}+\int_{\Omega^{\mathrm{f}, n+1}} \boldsymbol{\sigma}\left(\boldsymbol{u}^{n+1}, p^{n+1}\right): \boldsymbol{\nabla} \boldsymbol{v}^{\mathrm{f}}+\int_{\Omega^{\mathrm{f}, n+1}} q \operatorname{div} \boldsymbol{u}^{n+1} \\
+\frac{2 \rho^{\mathrm{s}}}{\tau^{2}} \int_{\Omega^{\mathrm{s}}}\left(\boldsymbol{d}^{n+1}-\boldsymbol{d}^{\mathrm{n}}-\tau \dot{\boldsymbol{d}}^{n}\right) \cdot \boldsymbol{v}^{\mathrm{s}}+\int_{\Omega^{\mathrm{s}}} \boldsymbol{\Pi}^{n+\frac{1}{2}}: \nabla \boldsymbol{v}_{\mathrm{s}} \\
=-\int_{\Gamma_{\text {out }}^{\mathrm{s}}} p_{\text {out }}\left(t_{n+1}\right) \boldsymbol{v}^{\mathrm{f}} \cdot \boldsymbol{n}
\end{array}
$$

for all $\left(\widehat{\boldsymbol{v}}^{\mathrm{f}}, \boldsymbol{v}^{\mathrm{s}}, \widehat{q}\right) \in \boldsymbol{H}_{\Gamma^{\text {in }}}^{1}\left(\Omega^{\mathrm{f}}\right) \times \boldsymbol{H}_{\Gamma^{\mathrm{d}}}^{1}\left(\Omega^{\mathrm{s}}\right) \times L^{2}\left(\Omega^{\mathrm{f}}\right)$ with $\left.\widehat{\boldsymbol{v}}^{\mathrm{f}}\right|_{\Sigma}=\left.\boldsymbol{v}^{\mathrm{s}}\right|_{\Sigma}$. 
Partitioned methods for the numerical solution of (17) typically stem from a domain-decomposition reformulation of this problem. Let $\widehat{L}^{\mathrm{f}}: \boldsymbol{H}^{\frac{1}{2}}(\Sigma) \rightarrow$ $\boldsymbol{H}_{\Gamma^{\text {in }} \cup \Gamma^{\text {out }}}^{1}\left(\Omega^{\mathrm{f}}\right)$ be a given continuous linear lift operator and consider the following splitting of the test functions space

$$
\begin{array}{r}
\left\{\left(\widehat{\boldsymbol{v}}^{\mathrm{f}}, \boldsymbol{v}^{\mathrm{s}}\right) \in \boldsymbol{H}_{\Gamma^{\text {in }}}^{1}\left(\Omega^{\mathrm{f}}\right) \times \boldsymbol{H}_{\Gamma^{\mathrm{d}}}^{1}\left(\Omega^{\mathrm{s}}\right):\left.\widehat{\boldsymbol{v}}^{\mathrm{f}}\right|_{\Sigma}=\left.\boldsymbol{v}^{\mathrm{s}}\right|_{\Sigma}\right\}=\left\{\left(\widehat{\boldsymbol{v}}^{\mathrm{f}}, \mathbf{0}\right): \widehat{\boldsymbol{v}}^{\mathrm{f}} \in \boldsymbol{H}_{\Gamma^{\text {in }} \cup \Sigma}^{1}\left(\Omega^{\mathrm{f}}\right)\right\} \\
\oplus\left\{\left(\widehat{L}^{\mathrm{f}}\left(\left.\boldsymbol{v}^{\mathrm{s}}\right|_{\Sigma}\right), \boldsymbol{v}^{\mathrm{s}}\right): \widehat{\boldsymbol{v}}^{\mathrm{s}} \in \boldsymbol{H}_{\Gamma^{\mathrm{d}}}^{1}\left(\Omega^{\mathrm{s}}\right)\right\} .
\end{array}
$$

By applying this decomposition to (17) we recover the following equivalent formulation, involving two coupled subproblems:

$$
\left\{\begin{aligned}
\mathcal{F}\left(\boldsymbol{d}^{\mathrm{f}, n+1}, \widehat{\boldsymbol{u}}^{n+1}, \widehat{p}^{n+1}, \boldsymbol{\gamma}^{n+1}\right) & =0, \\
\mathcal{S}\left(\boldsymbol{d}^{n+1}, \boldsymbol{\mu}^{n+1}\right) & =0
\end{aligned}\right.
$$

where $\left.\gamma^{n+1} \stackrel{\text { def }}{=} \boldsymbol{d}^{n+1}\right|_{\Sigma}$ is the interface displacement and $\boldsymbol{\mu}^{n+1} \stackrel{\text { def }}{=}$ $\mathcal{R}^{\mathrm{f}}\left(\boldsymbol{d}^{\mathrm{f}, n+1}, \widehat{\boldsymbol{u}}^{n+1}, \widehat{p}^{n+1}\right)$ the variationally consistent representation of the fluid stress at the interface (whose expression is given below). In short, equation $(18)_{1}$ ensures the fluid balance subjected to the interface displacement $\gamma^{n+1}$, whereas $(18)_{2}$ enforces the solid balance subjected to the interface fluid stress $\boldsymbol{\mu}^{n+1}$.

The fluid operator

$$
\begin{aligned}
\mathcal{F}: \boldsymbol{H}_{\Gamma^{\text {in }} \cup \Gamma^{\text {out }}}^{1}\left(\Omega^{\mathrm{f}}\right) \times \boldsymbol{H}^{1}\left(\Omega^{\mathrm{f}}\right) \times L^{2}\left(\Omega^{\mathrm{f}}\right) \times \boldsymbol{H}^{\frac{1}{2}}(\Sigma) \\
\rightarrow\left(\boldsymbol{H}_{\Gamma^{\text {in }} \cup \Sigma}^{1}\left(\Omega^{\mathrm{f}}\right) \times L^{2}\left(\Omega^{\mathrm{f}}\right) \times \boldsymbol{L}^{2}\left(\Gamma^{\text {in }} \cup \Sigma\right) \times \boldsymbol{L}^{2}\left(\Omega^{\mathrm{f}}\right)\right)^{\prime},
\end{aligned}
$$

is defined as

$$
\begin{aligned}
& \left\langle\mathcal{F}\left(\boldsymbol{d}^{\mathrm{f}}, \widehat{\boldsymbol{u}}, \widehat{p}, \boldsymbol{\gamma}\right),\left(\widehat{\boldsymbol{v}}^{\mathrm{f}}, \widehat{q}, \boldsymbol{\xi}, \boldsymbol{\zeta}\right)\right\rangle \stackrel{\text { def }}{=} \frac{\rho^{\mathrm{f}}}{\tau}\left(\int_{\Omega^{\mathrm{f}}\left(\boldsymbol{d}^{\mathrm{f}}\right)} \boldsymbol{u} \cdot \boldsymbol{v}^{\mathrm{f}}-\int_{\Omega^{\mathrm{f}, n}} \boldsymbol{u}^{n} \cdot \boldsymbol{v}^{\mathrm{f}}\right) \\
& \quad+\rho^{\mathrm{f}} \int_{\Omega^{\mathrm{f}}\left(\boldsymbol{d}^{\mathrm{f}}\right)}\left(\boldsymbol{u}-\boldsymbol{w}\left(\boldsymbol{d}^{\mathrm{f}}\right)\right) \cdot \boldsymbol{\nabla} \boldsymbol{u} \cdot \boldsymbol{v}^{\mathrm{f}}-\rho^{\mathrm{f}} \int_{\Omega^{\mathrm{f}}\left(\boldsymbol{d}^{\mathrm{f}}\right)}\left(\operatorname{div} \boldsymbol{w}\left(\boldsymbol{d}^{\mathrm{f}}\right)\right) \boldsymbol{u} \cdot \boldsymbol{v}^{\mathrm{f}} \\
& \quad+\int_{\Omega^{\mathrm{f}}\left(\boldsymbol{d}^{\mathrm{f}}\right)} \boldsymbol{\sigma}(\boldsymbol{u}, p): \boldsymbol{\nabla} \boldsymbol{v}^{\mathrm{f}}+\int_{\Omega^{\mathrm{f}}\left(\boldsymbol{d}^{\mathrm{f}}\right)} q \operatorname{div} \boldsymbol{v}^{\mathrm{f}}+\int_{\Gamma_{\text {out }}} p_{\text {out }}\left(t_{n+1}\right) \boldsymbol{v}^{\mathrm{f}} \cdot \boldsymbol{n} \\
& +\int_{\Omega^{\mathrm{f}}}\left(\boldsymbol{d}^{\mathrm{f}}-\operatorname{Ext}(\gamma)\right) \cdot \boldsymbol{\zeta}+\int_{\Sigma}\left(\widehat{\boldsymbol{u}}-\partial_{\tau} \boldsymbol{\gamma}\right) \cdot \boldsymbol{\xi}+\int_{\Gamma^{\text {in }}}\left(\boldsymbol{u}-\boldsymbol{u}_{\text {in }}\left(t_{n+1}\right)\right) \cdot \boldsymbol{\xi}
\end{aligned}
$$

for all $\left(\widehat{\boldsymbol{v}}^{\mathrm{f}}, \widehat{q}, \boldsymbol{\xi}, \boldsymbol{\zeta}\right) \in \boldsymbol{H}_{\Gamma^{\text {in }} \cup \Sigma}^{1}\left(\Omega^{\mathrm{f}}\right) \times L^{2}\left(\Omega^{\mathrm{f}}\right) \times \boldsymbol{L}^{2}\left(\Gamma^{\mathrm{in}} \cup \Sigma\right) \times \boldsymbol{L}^{2}\left(\Omega^{\mathrm{f}}\right)$. Here, we have used the notations $\widehat{\boldsymbol{w}}\left(\boldsymbol{d}^{\mathrm{f}}\right) \stackrel{\text { def }}{=}\left(\boldsymbol{d}^{\mathrm{f}}-\boldsymbol{d}^{\mathrm{f}, n}\right) / \tau, \partial_{\tau} \boldsymbol{\gamma} \stackrel{\text { def }}{=}\left(\boldsymbol{\gamma}-\boldsymbol{\gamma}^{n}\right) / \tau$ and $\Omega^{\mathrm{f}}\left(\boldsymbol{d}^{\mathrm{f}}\right) \stackrel{\text { def }}{=}\left(\boldsymbol{I}_{\Omega^{\mathrm{f}}}+\boldsymbol{d}^{\mathrm{f}}\right)\left(\Omega^{\mathrm{f}}\right)$.

The interface fluid residual operator $\mathcal{R}^{\mathrm{f}}: \boldsymbol{H}^{1}\left(\Omega^{\mathrm{f}}\right) \times \boldsymbol{H}^{1}\left(\Omega^{\mathrm{f}}\right) \times L^{2}\left(\Omega^{\mathrm{f}}\right) \rightarrow$ $\boldsymbol{H}^{-\frac{1}{2}}(\Sigma)$ is then defined by

$$
\left\langle\mathcal{R}^{\mathrm{f}}\left(\boldsymbol{d}^{\mathrm{f}}, \widehat{\boldsymbol{u}}, \widehat{p}\right), \boldsymbol{\lambda}\right\rangle \stackrel{\text { def }}{=}\left\langle\mathcal{F}\left(\boldsymbol{d}^{\mathrm{f}}, \widehat{\boldsymbol{u}}, \widehat{p}, \boldsymbol{\gamma}\right),\left(\widehat{L}^{\mathrm{f}} \boldsymbol{\lambda}, 0, \mathbf{0}, \mathbf{0}\right)\right\rangle
$$

for all $\boldsymbol{\lambda} \in \boldsymbol{H}^{\frac{1}{2}}(\Sigma)$. 
Remark 5 Note that the test function $\widehat{\boldsymbol{v}}^{\mathrm{f}}$ in (19) vanishes on the boundary $\Gamma^{\text {in }} \cup \Sigma$, so that Dirichlet boundary conditions are strongly imposed. The last two terms of (19) are not included in practice in the variational formulation. They have been incorporated in the definition of the fluid operator in order to facilitate the presentation. The same observation applies to the third last term.

Similarly, the solid operator

$$
\mathcal{S}: \boldsymbol{H}_{\Gamma^{\mathrm{d}}}^{1}\left(\Omega^{\mathrm{s}}\right) \times \boldsymbol{H}^{-\frac{1}{2}}(\Sigma) \rightarrow\left(\boldsymbol{H}_{\Gamma^{\mathrm{d}}}^{1}\left(\Omega^{\mathrm{s}}\right)\right)^{\prime},
$$

is defined as

$$
\begin{array}{r}
\left\langle\mathcal{S}(\boldsymbol{d}, \boldsymbol{\mu}), \boldsymbol{v}^{\mathrm{s}}\right\rangle \stackrel{\text { def }}{=} \frac{2 \rho^{\mathrm{s}}}{\tau^{2}} \int_{\Omega^{\mathrm{s}}}\left(\boldsymbol{d}-\boldsymbol{d}^{\mathrm{n}}-\tau \dot{\boldsymbol{d}}^{n}\right) \cdot \boldsymbol{v}^{\mathrm{s}}+\frac{1}{2} \int_{\Omega^{\mathrm{s}}}(\boldsymbol{\Pi}(\boldsymbol{d})+ \\
\left.+\boldsymbol{\Pi}\left(\boldsymbol{d}^{n}\right)\right): \nabla \boldsymbol{v}^{\mathrm{s}} \\
+\left\langle\boldsymbol{\mu},\left.\boldsymbol{v}^{\mathrm{s}}\right|_{\Sigma}\right\rangle
\end{array}
$$

for all $\boldsymbol{v}^{\mathrm{s}} \in \boldsymbol{H}_{\Gamma^{\mathrm{d}}}^{1}\left(\Omega^{\mathrm{s}}\right)$.

Finally, problem (18) can be reformulated as an interface problem in terms of the nonlinear fluid and solid Steklov-Poincaré operators (see [36]). The fluid Steklov-Poincaré operator $S^{\mathrm{f}}: \boldsymbol{H}^{\frac{1}{2}}(\Sigma) \rightarrow \boldsymbol{H}^{-\frac{1}{2}}(\Sigma)$ (also called DirichletNeumann map) is defined by

$$
S^{\mathrm{f}}(\boldsymbol{\gamma}) \stackrel{\text { def }}{=} \mathcal{R}^{\mathrm{f}}\left(\boldsymbol{d}^{\mathrm{f}}(\boldsymbol{\gamma}), \widehat{\boldsymbol{u}}(\boldsymbol{\gamma}), \widehat{p}(\gamma)\right) \quad \forall \boldsymbol{\gamma} \in \boldsymbol{H}^{\frac{1}{2}}(\Sigma),
$$

where $\left(\boldsymbol{d}^{\mathrm{f}}(\boldsymbol{\gamma}), \widehat{\boldsymbol{u}}(\boldsymbol{\gamma}), \widehat{p}(\boldsymbol{\gamma})\right)$ is the solution of the Dirichlet fluid subproblem:

$$
\mathcal{F}\left(\boldsymbol{d}^{\mathrm{f}}(\boldsymbol{\gamma}), \widehat{\boldsymbol{u}}(\boldsymbol{\gamma}), \widehat{p}(\boldsymbol{\gamma}), \boldsymbol{\gamma}\right)=0
$$

In other words, $S^{\mathrm{f}}(\boldsymbol{\gamma})$ gives the interface fluid stress associated to the displacement $\gamma$ of the interface. Analogously, the nonlinear solid inverse SteklovPoincaré operator $S^{\mathrm{s}}: \boldsymbol{H}^{-\frac{1}{2}}(\Sigma) \rightarrow \boldsymbol{H}^{\frac{1}{2}}(\Sigma)$ (also called Neumann-Dirichlet map) is given by

$$
S^{\mathrm{S}}(\boldsymbol{\mu})=\left.\boldsymbol{d}(\boldsymbol{\mu})\right|_{\Sigma} \quad \forall \boldsymbol{\mu} \in \boldsymbol{H}^{-\frac{1}{2}}(\Sigma),
$$

where $\boldsymbol{d}(\boldsymbol{\mu})$ is the solution of the Neumann solid subproblem:

$$
\mathcal{S}(\boldsymbol{d}(\boldsymbol{\mu}), \boldsymbol{\mu})=0 .
$$

From the above definitions, it follows that problem (18) (or, equivalently, (17)) is equivalent to the following interface problem: find $\gamma^{n+1} \in \boldsymbol{H}^{\frac{1}{2}}(\Sigma)$ such that

$$
S^{\mathrm{s}}\left(S^{\mathrm{f}}\left(\gamma^{n+1}\right)\right)=\gamma^{n+1} .
$$

This equation is the so-called interface Dirichlet-Neumann formulation of (17). The composition of (24) with the inverse operator $\left(S^{\mathrm{s}}\right)^{-1}$, gives rise to the so-called Steklov-Poincaré equation (see [36]):

$$
\left(S^{\mathrm{s}}\right)^{-1}\left(\gamma^{n+1}\right)-S^{\mathrm{f}}\left(\gamma^{n+1}\right)=0 .
$$

Remark 6 For the sake of conciseness, we have limited the presentation to the time semi-discrete problem (17). Nevertheless, the discussion also applies to the fully discrete case, for instance, after space discretization of (17) with finite elements. 


\subsection{Partitioned solution methods}

These methods are generally based on the application of a particular nonlinear iterative method to the interface formulations (23) or (24). In this subsection we discuss some iterative procedures applied to (23). Some solution methods for the non-linear problem (24) are introduced in [36]. Alternative partitioned procedures, based on Robin-Neumann transmission conditions, have been recently introduced in $[9,10]$.

The formulation (23) reduces problem (18) to the determination of a fixed point of the Dirichlet-Neumann operator $S^{\mathrm{s}} \circ S^{\mathrm{f}}$. This motivates the use of fixedpoint (e.g., non-linear Richardson) based iterations, as shown in Algorithm 3 (see, e.g., $[91,92,85,96,35,107,78]$ ), where $\omega_{k} \in(0,1]$ is a given relaxation parameter which is chosen in order to guarantee convergence. At the fully

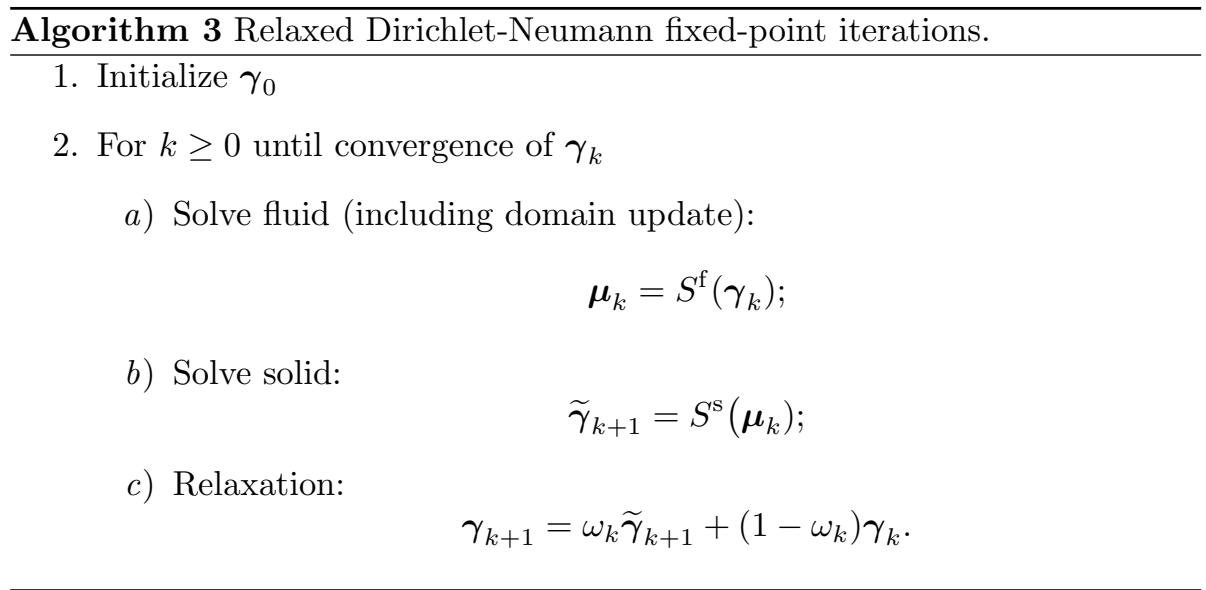

discrete level (i.e., after discretization in space), an expression for this parameter (which significantly improves the convergence) is given by the following multidimensional Aitken's formula (see [92, 35, 78]):

$$
\omega_{k}=\frac{\left(\gamma_{k}-\gamma_{k-1}\right) \cdot\left(\widetilde{\gamma}_{k+1}-\gamma_{k}+\widetilde{\gamma}_{k}-\gamma_{k-1}\right)}{\left|\widetilde{\gamma}_{k+1}-\gamma_{k}+\widetilde{\gamma}_{k}-\gamma_{k-1}\right|^{2}}, \quad k \geq 1 .
$$

Algorithm 3 can be considered as the simplest way of solving implicit coupling in a partitioned fashion: existing fluid and solid solvers (possibly black-box) can be straightforwardly coupled, without significant modifications of the two solvers. The method, however, may suffer from a poor convergence behavior, which is dictated by the amount of added-mass effect in the system. Indeed, increased relaxation is required when the solid density decreases or the domain length increases, which can compromise efficiency in real applications. Theoretical explanations of this issue have been reported in [25] using a simplified model (see also [85, 34, 74, 31]). The limitations of Algorithm 3 have led to the development of new variants: for instance, based on the use of transpiration techniques [37], reduced order models [116], vector extrapolation 
[79], interface artificial compressibility [104, 73, 33], and Robin-Neumann coupling [9]. It is worth noticing that these last two variants achieve convergence without the need of relaxation and have a low sensitivity to the added-mass effect.

Alternatively, one can apply a Newton based method to (23), for a fast convergence towards the solution (see, e.g., $[59,60,32,49]$ ). This yields Algorithm 4, which involves the Jacobian $D_{\gamma} R$ of the coupled operator

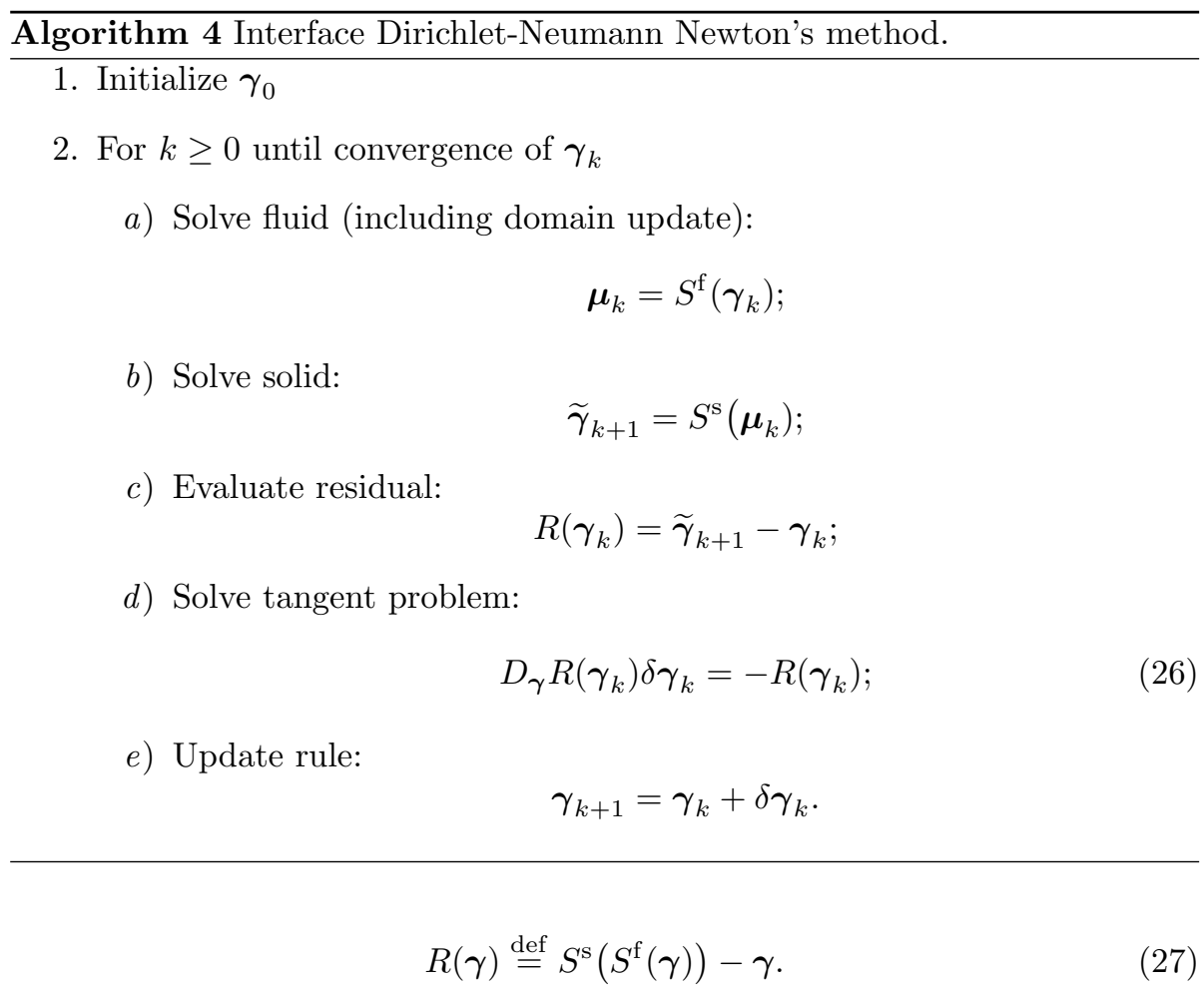

In practice, the linearized fluid-structure problem (26) is solved using an operator-free (Krylov) iterative method, as GMRES, which only requires repeated evaluations of $D_{\gamma} R(\gamma)$ against given interface displacements $\boldsymbol{\lambda}$. In other words, the Jacobian operator $D_{\gamma} R(\gamma)$ is not explicitly needed.

Approximate evaluations of $D_{\boldsymbol{\gamma}} R(\boldsymbol{\gamma}) \boldsymbol{\lambda}$ (or resolutions of $(26)$ ) lead to the socalled inexact (or quasi-) Newton methods (see, e.g., [59, 78, 32]). For instance, we can use as an approximation the difference quotient

$$
D_{\gamma} R(\boldsymbol{\gamma}) \boldsymbol{\lambda} \approx \frac{1}{\epsilon}(R(\boldsymbol{\gamma}+\epsilon \boldsymbol{\lambda})-R(\boldsymbol{\gamma})),
$$

with $\epsilon>0$ a given small enough parameter (see, e.g., [19]). Note that this approach facilitates the use of black-box solvers (as Algorithm 3), since (28) only requires residual evaluations. Nevertheless, as noticed in [59, Remark 5.1] (see also [78]), such a strategy may lead to inefficient Newton iterations. 
Using the chain rule, we have

$$
D_{\boldsymbol{\gamma}} R(\boldsymbol{\gamma}) \boldsymbol{\lambda}=D_{\boldsymbol{\mu}} S^{\mathrm{s}}\left(S^{\mathrm{f}}(\boldsymbol{\gamma})\right) D_{\boldsymbol{\gamma}} S^{\mathrm{f}}(\boldsymbol{\gamma}) \boldsymbol{\lambda}-\boldsymbol{\lambda}
$$

so that the exact evaluation of $D_{\gamma} R(\boldsymbol{\gamma}) \boldsymbol{\lambda}$ can be split into the following three sequential steps:

(i) Solve the linearized fluid subproblem:

$$
\zeta=D_{\gamma} S^{\mathrm{f}}(\gamma) \boldsymbol{\lambda}
$$

(ii) Solve the linearized solid subproblem:

$$
\boldsymbol{\eta}=D_{\boldsymbol{\mu}} S^{\mathrm{s}}\left(S^{\mathrm{f}}(\gamma)\right) \boldsymbol{\zeta}
$$

(iii) Update: $D_{\boldsymbol{\gamma}} R(\boldsymbol{\gamma}) \boldsymbol{\lambda}=\boldsymbol{\eta}-\boldsymbol{\lambda}$.

Steps (i) and (ii) require the linearized versions of the fluid and solid solvers. Note that step (ii) is standard in solid solvers. Step (i), on the contrary, is non-standard and usually not available in most fluid solvers. For this reason, this step is usually approximated (see, e.g., $[59,60]$ ).

In the rest of this subsection, we present the procedure proposed in [49] for the evaluation of $D_{\gamma} R(\gamma) \boldsymbol{\lambda}$, based on shape-derivative calculus (see [108, 1]). By derivation of (21) with respect to $\gamma$ in the direction $\boldsymbol{\lambda}$, we have

$$
\begin{array}{r}
\left\langle D_{\gamma} S^{\mathrm{f}}(\boldsymbol{\gamma}) \boldsymbol{\lambda}, \boldsymbol{\theta}\right\rangle \stackrel{\text { def }}{=}-\left\langle D_{\boldsymbol{d}^{\mathrm{f}}} \mathcal{F}\left(\boldsymbol{d}^{\mathrm{f}}(\boldsymbol{\gamma}), \widehat{\boldsymbol{u}}(\boldsymbol{\gamma}), \widehat{p}(\boldsymbol{\gamma}), \boldsymbol{\gamma}\right) \delta \widehat{\boldsymbol{d}}^{\mathrm{f}},\left(\widehat{L}^{\mathrm{f}} \boldsymbol{\theta}, 0, \mathbf{0}, \mathbf{0}\right)\right\rangle \\
-\left\langle D_{(\widehat{\boldsymbol{u}}, \widehat{p}, \boldsymbol{\gamma})} \mathcal{F}\left(\boldsymbol{d}^{\mathrm{f}}(\boldsymbol{\gamma}), \widehat{\boldsymbol{u}}(\boldsymbol{\gamma}), \widehat{p}(\boldsymbol{\gamma}), \boldsymbol{\gamma}\right)(\delta \widehat{\boldsymbol{u}}, \delta \widehat{p}, \boldsymbol{\lambda}),\left(\widehat{L}^{\mathrm{f}} \boldsymbol{\theta}, 0, \mathbf{0}, \mathbf{0}\right)\right\rangle
\end{array}
$$

for all $\boldsymbol{\theta} \in \boldsymbol{H}^{\frac{1}{2}}(\Sigma)$ and with the notations $\delta \widehat{\boldsymbol{d}}^{\mathrm{f}} \stackrel{\text { def }}{=} D_{\boldsymbol{\gamma}} \boldsymbol{d}^{\mathrm{f}}(\boldsymbol{\gamma}) \boldsymbol{\lambda}, \delta \widehat{\boldsymbol{u}} \stackrel{\text { def }}{=} D_{\boldsymbol{\gamma}} \widehat{\boldsymbol{u}}(\boldsymbol{\gamma}) \boldsymbol{\lambda}$ and $\delta \widehat{p} \stackrel{\text { def }}{=} D_{\gamma} \widehat{p}(\gamma) \boldsymbol{\lambda}$. While the second derivative in the right hand-side of (31) is standard (e.g., a classical Fréchet derivative), the cross-Jacobian $D_{\boldsymbol{d}^{\mathrm{f}}} \mathcal{F}$ requires shape-derivative calculus, since it involves the derivation with respect to $\boldsymbol{d}^{\mathrm{f}}$ of Eulerian integrals over $\Omega\left(\boldsymbol{d}^{\mathrm{f}}\right)$. This yields the following expression (see [49] for details):

$$
\begin{gathered}
\left\langle D_{\boldsymbol{d}^{\mathrm{f}}} \mathcal{F}\left(\boldsymbol{d}^{\mathrm{f}}, \widehat{\boldsymbol{u}}, \widehat{p}, \gamma\right) \delta \widehat{\boldsymbol{d}},(\widehat{\boldsymbol{v}}, \widehat{q}, \boldsymbol{\xi}, \boldsymbol{\zeta})\right\rangle=\frac{1}{\tau} \int_{\Omega\left(\boldsymbol{d}^{\mathrm{f}}\right)} \rho^{\mathrm{f}}\left(\operatorname{div} \delta \boldsymbol{d}^{\mathrm{f}}\right) \boldsymbol{u} \cdot \boldsymbol{v} \\
+\int_{\Omega\left(\boldsymbol{d}^{\mathrm{f}}\right)} \rho^{\mathrm{f}}\left(\operatorname{div} \delta \boldsymbol{d}^{\mathrm{f}}\right)\left(\boldsymbol{u}-\boldsymbol{w}\left(\boldsymbol{d}^{\mathrm{f}}\right)\right) \cdot \boldsymbol{\nabla} \cdot \boldsymbol{v}-\int_{\Omega\left(\boldsymbol{d}^{\mathrm{f}}\right)} \rho^{\mathrm{f}}\left[\boldsymbol{\nabla} \boldsymbol{u} \boldsymbol{\nabla} \delta \boldsymbol{d}^{\mathrm{f}}\left(\boldsymbol{u}-\boldsymbol{w}\left(\boldsymbol{d}^{\mathrm{f}}\right)\right)\right] \cdot \boldsymbol{v} \\
-\frac{1}{\tau} \int_{\Omega\left(\boldsymbol{d}^{\mathrm{f}}\right)} \rho^{\mathrm{f}} \delta \boldsymbol{d}^{\mathrm{f}} \cdot \boldsymbol{\nabla} \boldsymbol{u} \cdot \boldsymbol{v}+\int_{\Omega\left(\boldsymbol{d}^{\mathrm{f}}\right)} \boldsymbol{\sigma}(\boldsymbol{u}, p)\left[\boldsymbol{I} \operatorname{div} \delta \boldsymbol{d}^{\mathrm{f}}-\left(\boldsymbol{\nabla} \delta \boldsymbol{d}^{\mathrm{f}}\right)^{\mathrm{T}}\right]: \nabla \boldsymbol{v} \\
-\int_{\Omega\left(\boldsymbol{d}^{\mathrm{f}}\right)} \mu\left[\boldsymbol{\nabla} \boldsymbol{\nabla} \delta \boldsymbol{d}^{\mathrm{f}}+\left(\boldsymbol{\nabla} \delta \boldsymbol{d}^{\mathrm{f}}\right)^{\mathrm{T}}(\boldsymbol{\nabla} \boldsymbol{u})^{\mathrm{T}}\right]: \nabla \boldsymbol{v} \\
-\int_{\Omega\left(\boldsymbol{d}^{\mathrm{f}}\right)} q \operatorname{div}\left\{\boldsymbol{u}\left[\boldsymbol{I} \operatorname{div} \delta \boldsymbol{d}^{\mathrm{f}}-\left(\boldsymbol{\nabla} \delta \boldsymbol{d}^{\mathrm{f}}\right)^{\mathrm{T}}\right]\right\}+\int_{\Omega} \delta \widehat{\boldsymbol{d}}^{\mathrm{f}} \cdot \boldsymbol{\zeta}, \quad(32)
\end{gathered}
$$


for all $(\widehat{\boldsymbol{v}}, \widehat{q}, \boldsymbol{\xi}, \boldsymbol{\zeta}) \in \boldsymbol{H}_{\Gamma^{\text {in }} \cup \Sigma}^{1}\left(\Omega^{\mathrm{f}}\right) \times L^{2}\left(\Omega^{\mathrm{f}}\right) \times \boldsymbol{L}^{2}\left(\Gamma^{\mathrm{in}} \cup \Sigma\right) \times \boldsymbol{L}^{2}\left(\Omega^{\mathrm{f}}\right)$. Note that the above terms (see also (see $[38,15]$ ) are not standard in a fluid research code, which explains why these terms have been usually neglected, or approximated by finite differences (see, e.g., [113, 89, 70]).

On the other hand, $\delta \widehat{\boldsymbol{d}}^{\mathrm{f}}, \delta \widehat{\boldsymbol{u}}$ and $\delta \widehat{p}$ can be obtained by implicit derivation of (22). This yields

$$
\delta \widehat{\boldsymbol{d}}^{\mathrm{f}}=D_{\gamma} \operatorname{Ext}(\boldsymbol{\gamma}) \boldsymbol{\lambda},
$$

and $(\delta \widehat{\boldsymbol{u}}, \delta \widehat{p})$ solve the linearized fluid subproblem:

$$
\begin{aligned}
\left\langle D_{(\widehat{\boldsymbol{u}}, \widehat{p})} \mathcal{F}\left(\boldsymbol{d}^{\mathrm{f}}(\boldsymbol{\gamma}), \widehat{\boldsymbol{u}}(\boldsymbol{\gamma}), \widehat{p}(\boldsymbol{\gamma}), \boldsymbol{\gamma}\right)(\delta \widehat{\boldsymbol{u}}, \delta \widehat{p}),(\widehat{\boldsymbol{v}}, \widehat{q}, \mathbf{0}, \mathbf{0})\right\rangle \\
\quad=-\left\langle D_{\boldsymbol{d}^{\mathrm{f}}} \mathcal{F}\left(\boldsymbol{d}^{\mathrm{f}}(\boldsymbol{\gamma}), \widehat{\boldsymbol{u}}(\boldsymbol{\gamma}), \widehat{p}(\boldsymbol{\gamma}), \boldsymbol{\gamma}\right) \delta \widehat{\boldsymbol{d}}^{\mathrm{f}},(\widehat{\boldsymbol{v}}, \widehat{q}, \mathbf{0}, \mathbf{0})\right\rangle
\end{aligned}
$$

for all $(\widehat{\boldsymbol{v}}, \widehat{q}) \in \boldsymbol{H}_{\Gamma^{\text {in }} \cup \Sigma}^{1}\left(\Omega^{\mathrm{f}}\right) \times L^{2}\left(\Omega^{\mathrm{f}}\right)$, with the boundary conditions $\left.\delta \widehat{\boldsymbol{u}}\right|_{\Sigma}=\boldsymbol{\lambda} / \tau$, $\left.\delta \widehat{\boldsymbol{u}}\right|_{\Gamma^{\text {in }}}=\mathbf{0}$.

In summary, for each interface displacement $\boldsymbol{\lambda}$, the sensitivity $D_{\gamma} S_{\mathrm{f}}(\boldsymbol{\gamma}) \boldsymbol{\lambda}$ can be evaluated as follows:

(i) Compute the fluid domain displacement sensitivity $\delta \widehat{\boldsymbol{d}}^{\mathrm{f}}$ from (33);

(ii) Compute the fluid sensitivities $(\delta \widehat{\boldsymbol{u}}, \delta \widehat{p})$ from (34) and (32);

(iii) Evaluate $D_{\gamma} S^{\mathrm{f}}(\boldsymbol{\gamma}) \boldsymbol{\lambda}$ from (31) and (32).

Remark 7 Note that each operator-free evaluation (29) (one per GMRES iteration in (26)) requires the resolution of the linearized fluid subproblem (34) and the linearized solid sub-problem (30).

We conclude this subsection with a few numerical illustrations from [49], involving the coupling of the ALE Navier-Stokes equations with the linear elasticity equations. The reported results correspond to the simulation of a pressure wave propagation in a compliant straight vessel $($ see $[52,59])$. The following procedures are compared:

- FP-Aitken: Algorithm 3 with Aitken's dynamic relaxation (25);

- Newton: Algorithm 4 with exact Jacobian evaluation described above;

- Inexact-Newton: Algorithm 4 with the inexact Jacobian evaluation obtained by neglecting the cross-Jacobian $D_{\boldsymbol{d}^{\mathrm{f}}} \mathcal{F}$ (shape terms) in (34) and (32).

Figure 2 (left) reports the number of iterations per time-step, performed by each procedure. The superior convergence behavior of both Newton algorithms is clearly visible. Figure 2 (right) shows that both Newton algorithms are about 2 times faster than the fixed-point algorithm (see [49] for a detailed discussion). Note that the cost of each Newton iteration is higher than the cost of a fixedpoint iteration (see Remark 7). 


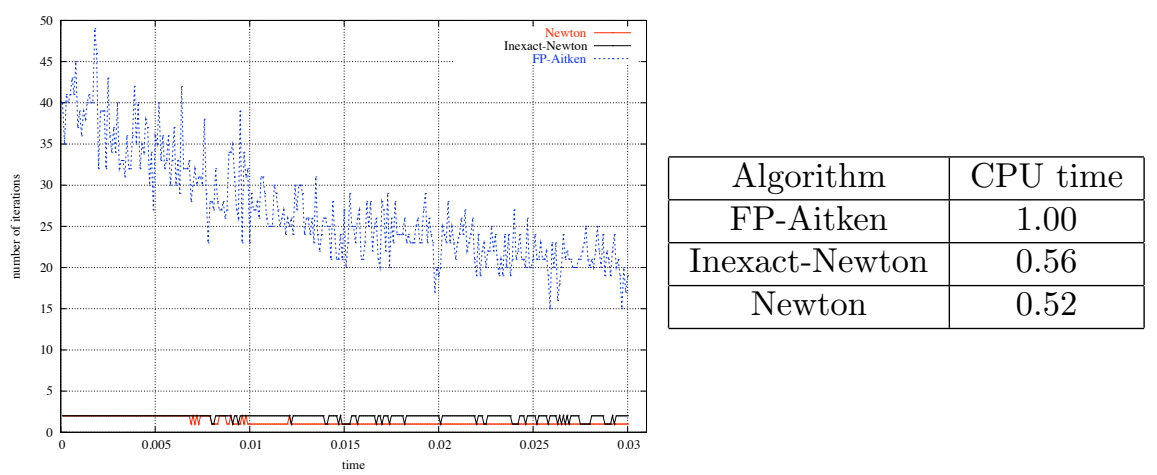

Figure 2: Left: number of non-linear iterations per time-step. Right: dimensionless elapsed CPU time $\left(\tau=10^{-4} \mathrm{~s}\right)$. From [49].

The impact of the exact Jacobian evaluations in Algorithm 4 can be highlighted by increasing the time-step size. Figure 3 (left), reports the number of iterations per time-step obtained with $\tau=10^{-3} \mathrm{~s}$. The fixed-point and inexact-Newton algorithms fail to converge after two time steps (the allowed maximum number of iterations is reached) whereas the exact Newton method converges and requires a low number of iterations. Figure 3 (right) shows the evolution of the residual during the iteration process in both Newton algorithms at the third time step. While the exact Newton only requires 3 iterations to reach the convergence threshold, the inexact-Newton algorithm is unable to reduce the residual.
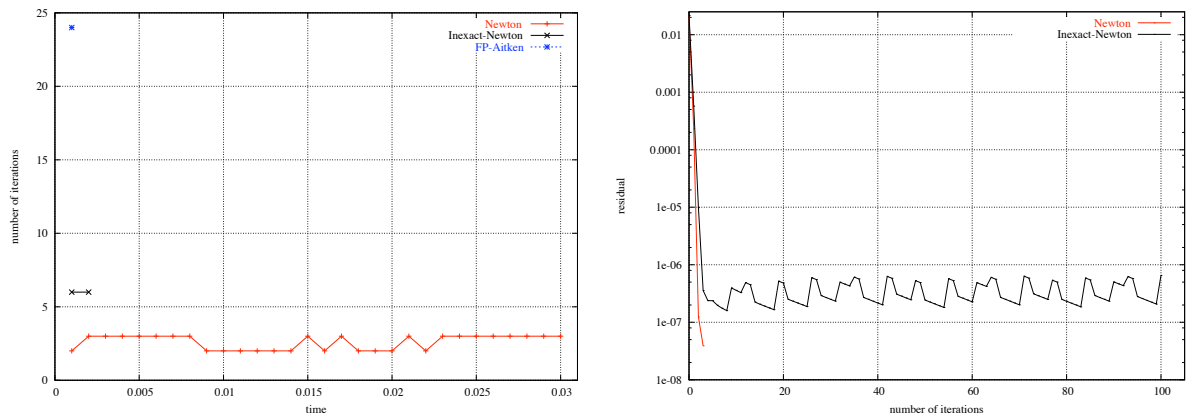

Figure 3: Left: number of non-linear iterations per time-step. Right: Newton residuals $\left(\tau=10^{-3} \mathrm{~s}\right)$. From [49].

Remark 8 Numerical evidence shows that the convergence of the GMRES iterations involved in (26) is sensitive to the amount of added-mass effect in the system (see [11]), whereas the number of Newton iterations is practically unaffected. The overall sensitivity is, however, remarkably lower than that of Algorithm 3 (see [11, §6.1]). Note that, for linear fluid and solid solvers, 
Algorithms 3 and 4 can be viewed as, Dirichlet-Neumann preconditioned, Richardson and GMRES iterations, respectively. Hence, the superiority of Algorithms 4. At last, let us mention that the added-mass sensitivity of the GMRES iterations can be reduced by using Robin-Neumann preconditioners, as proposed in [10].

\section{Semi-implicit coupling}

A first approach to reduce the computational complexity of implicit coupling consists in treating the fluid domain geometry explicitly (see, e.g., $[96,110,97,111])$. This corresponds to the following explicit-implicit treatment of (13):

$$
\begin{aligned}
& \boldsymbol{d}^{\mathrm{f}, n+1}=\operatorname{Ext}\left(\left.\boldsymbol{d}^{n}\right|_{\Sigma}\right), \quad \widehat{\boldsymbol{w}}^{n+1}=\partial_{\tau} \boldsymbol{d}^{\mathrm{f}, n+1}, \quad \Omega^{\mathrm{f}, n+1}=\left(\boldsymbol{I}_{\Omega^{\mathrm{f}}}+\boldsymbol{d}^{\mathrm{f}, n+1}\right)\left(\Omega^{\mathrm{f}}\right), \\
& \left\{\begin{array}{l}
\widehat{\boldsymbol{u}}^{n+1}=\partial_{\tau} \boldsymbol{d}^{n+1} \text { on } \Sigma, \\
\boldsymbol{\Pi}^{n+\frac{1}{2}} \boldsymbol{n}^{\mathrm{s}}=-J^{n+1} \widehat{\boldsymbol{\sigma}}\left(\boldsymbol{u}^{n+1}, p^{n+1}\right)\left(\boldsymbol{F}^{n+1}\right)^{-\mathrm{T}} \boldsymbol{n} \quad \text { on } \quad \Sigma,
\end{array}\right.
\end{aligned}
$$

which, combined with (14) and (15), yields the time-marching scheme detailed in Algorithm 5. Although not fully implicit, this scheme is strongly coupled since the transmission conditions $(13)_{2}$ and $(13)_{3}$ are treated implicitly via (36). As a result, the stability of Algorithm 5 is not compromised by the amount of added-mass effect in the system (see $[110,97,111]$ ).

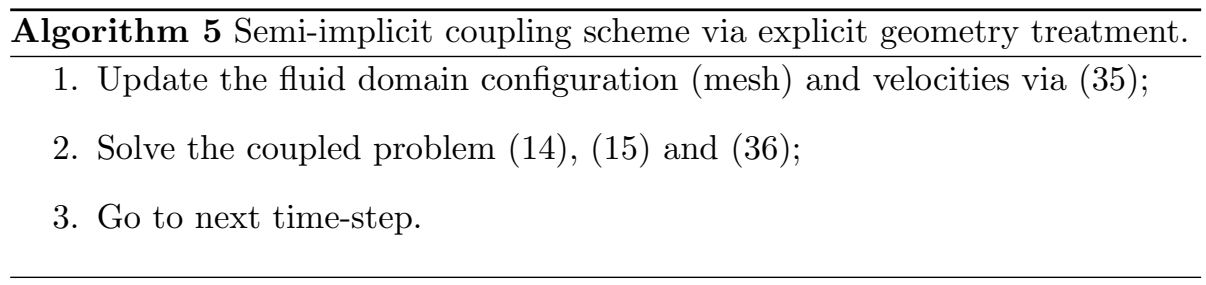

At each time-step, Algorithm 5 involves the resolution of the non-linear system (14), (15) and (36). This coupled problem enters the abstract framework of the previous section (by simply removing the unknown $\boldsymbol{d}^{\mathrm{f,n+1}}$ in (18)) and, therefore, can be solved by means of the partitioned procedures discussed therein. Note however that, due to the explicit treatment of the fluid geometry, the corresponding fluid operator $S^{f}$ does not involve the computation of the displacement $\boldsymbol{d}^{\mathrm{f}}$. Hence, the shape terms, involved in the cross-derivative $D_{\boldsymbol{d}^{\mathrm{f}}} \mathcal{F}$, are no longer needed for the exact evaluation of $D_{\gamma} S^{\mathrm{f}}$ in the tangent problem (26) of Algorithm 4.

\subsection{Projection-based semi-implicit coupling}

As mentioned above, Algorithm 5 is still a strongly coupled scheme, in the sense that $(13)_{2}$ and $(13)_{3}$ are exactly enforced at each time-step. In this subsection we present the alternative semi-implicit coupling scheme proposed in 
Algorithm 6 Semi-implicit coupling projection scheme ([48]).

1. Implicit step (pressure-structure coupling):

- Fluid projection sub-step:

$$
\left\{\begin{array}{rc}
\frac{\rho^{\mathrm{f}}}{\tau}\left(\boldsymbol{u}^{n+1}-\widetilde{\boldsymbol{u}}^{n}\right)+\nabla p^{n+1}=\mathbf{0} & \text { in } \quad \Omega^{\mathrm{f}, n} \\
\operatorname{div} \boldsymbol{u}^{n+1}=0 & \text { in } \quad \Omega^{\mathrm{f}, n} \\
\boldsymbol{u}^{n+1} \cdot \boldsymbol{n}=\boldsymbol{u}_{\mathrm{in}}\left(t_{n+1}\right) \cdot \boldsymbol{n} & \text { on } \quad \Gamma^{\mathrm{in}} \\
p^{n+1}=p_{\text {out }}\left(t_{n+1}\right) & \text { on } \quad \Gamma^{\text {out }} \\
\widehat{\boldsymbol{u}}^{n+1} \cdot \boldsymbol{n}=\partial_{\tau} \boldsymbol{d}^{n+1} \cdot \boldsymbol{n} & \text { on } \quad \Sigma
\end{array}\right.
$$

- Solid:

$$
\left\{\begin{array}{rcc}
\rho^{\mathrm{s}} \partial_{\tau} \dot{\boldsymbol{d}}^{n+1}-\operatorname{div} \boldsymbol{\Pi}^{n+\frac{1}{2}}=\mathbf{0} & \text { in } & \Omega^{\mathrm{s}} \\
\partial_{\tau} \boldsymbol{d}^{n+1}=\dot{\boldsymbol{d}}^{n+\frac{1}{2}} & \text { in } & \Omega^{\mathrm{s}} \\
\boldsymbol{d}^{n+1}=\mathbf{0} & \text { on } & \Gamma^{\mathrm{d}} \\
\boldsymbol{\Pi}^{n+\frac{1}{2}} \boldsymbol{n}^{\mathrm{s}}=\mathbf{0} & \text { on } & \Gamma^{\mathrm{n}} \\
\boldsymbol{\Pi}^{n+\frac{1}{2}} \boldsymbol{n}^{\mathrm{s}}=-J^{n} \widehat{\boldsymbol{\sigma}}\left(\widetilde{\boldsymbol{u}}^{n}, p^{n+1}\right)\left(\boldsymbol{F}^{n}\right)^{-\mathrm{T}} \boldsymbol{n} & \text { on } & \Sigma
\end{array}\right.
$$

2. Explicit step (viscous-structure coupling):

- Update fluid domain:

$$
\begin{aligned}
& \boldsymbol{d}^{\mathrm{f}, n+1}=\operatorname{Ext}\left(\left.\boldsymbol{d}^{n}\right|_{\Sigma}\right), \quad \widehat{\boldsymbol{w}}^{n+1}=\partial_{\tau} \boldsymbol{d}^{\mathrm{f}, n+1}, \\
& \Omega^{\mathrm{f}, n+1}=\left(\boldsymbol{I}_{\Omega^{\mathrm{f}}}+\boldsymbol{d}^{\mathrm{f}, n+1}\right)\left(\Omega^{\mathrm{f}}\right) .
\end{aligned}
$$

- Fluid viscous sub-step:

$$
\left\{\begin{aligned}
&\left.\rho^{\mathrm{f}} \frac{\widetilde{\boldsymbol{u}}^{n+1}-\boldsymbol{u}^{n+1}}{\tau}\right|_{\mathcal{A}}+\rho^{\mathrm{f}}\left(\widetilde{\boldsymbol{u}}^{n}-\boldsymbol{w}^{n+1}\right) \cdot \boldsymbol{\nabla} \widetilde{\boldsymbol{u}}^{n+1} \text { in } \quad \Omega^{\mathrm{f}, n+1}, \\
&-2 \mu \operatorname{div} \boldsymbol{\epsilon}\left(\widetilde{\boldsymbol{u}}^{n+1}\right)=\mathbf{0} \\
& \widetilde{\boldsymbol{u}}^{n+1}=\boldsymbol{u}_{\text {in }}\left(t_{n+1}\right) \text { on } \quad \Gamma^{\text {in }}, \\
& 2 \mu \boldsymbol{\epsilon}\left(\widetilde{\boldsymbol{u}}^{n+1}\right) \boldsymbol{n}^{\mathrm{f}}=\mathbf{0} \quad \text { on } \quad \Gamma^{\text {out }}, \\
& \widehat{\widehat{\boldsymbol{u}}}^{n+1}=\partial_{\tau} \boldsymbol{d}^{n+1} \text { on } \Sigma .
\end{aligned}\right.
$$

3. Go to next time-step. 
$[47,48]$. Though not strongly coupled, this scheme exhibits very good stability properties. Basically this scheme relies upon the three following ideas:

- the pressure-structure coupling is treated implicitly in order to avoid instabilities. This observation is motivated by the analysis reported in [25], which shows that explicit pressure-structure coupling yields a scheme whose stability is dictated by the amount of added-mass effect in the system;

- the remaining terms of the fluid equations (dissipation, convection and geometrical non-linearities) are explicitly coupled to the structure. This drastically reduces the cost of the coupling without compromising the overall stability of the scheme;

- this implicit-explicit coupling can be conveniently performed using a Chorin-Temam projection scheme (see, e.g, [112, 29, 63]) in the fluid. Indeed, at each time step we propose to couple implicitly the projection sub-step (carried out in a known fluid domain) with the structure, so accounting for the added-mass effect in an implicit way, while the ALEadvection-viscous sub-step is explicitly coupled.

The detailed steps of the semi-implicit coupling scheme proposed in [48] are given in Algorithm 6. Here, the scheme is presented in its (nonincremental) velocity-correction version and with a pressure-Darcy formulation of the projection step (see [63, Section 4.1]).

Remark 9 Alternatively, the projection step (37) can be formulated as the pressure-Poisson problem:

$$
\left\{\begin{array}{rcc}
-\Delta p^{n+1}=-\frac{\rho^{\mathrm{f}}}{\tau} \operatorname{div} \widetilde{\boldsymbol{u}}^{n} & \text { in } & \Omega^{\mathrm{f}, n}, \\
\partial_{\boldsymbol{n}} p^{n+1}=-\rho^{\mathrm{f}} \partial_{t} \boldsymbol{u}_{\mathrm{in}}\left(t_{n+1}\right) \cdot \boldsymbol{n} & \text { on } & \Gamma^{\mathrm{in}}, \\
p^{n+1}=p_{\text {out }}\left(t_{n+1}\right) & \text { on } & \Gamma^{\text {out }}, \\
\partial_{\boldsymbol{n}} p^{n+1}=-\frac{\rho^{\mathrm{f}}}{\tau}\left(\partial_{\tau} \boldsymbol{d}^{n+1}-\widetilde{\boldsymbol{u}}^{n}\right) \cdot \boldsymbol{n} & \text { on } & \Sigma^{n} .
\end{array}\right.
$$

Moreover, the divergence free velocity unknown $\boldsymbol{u}^{n+1}$ can then be eliminated in (40) via the relation

$$
\frac{\rho^{\mathrm{f}}}{\tau} \boldsymbol{u}^{n+1}=\frac{\rho^{\mathrm{f}}}{\tau} \widetilde{\boldsymbol{u}}^{n}-\nabla p^{n+1} \quad \text { in } \quad \Omega^{\mathrm{f}, n} .
$$

Note that step 2 of Algorithm 6 is performed only once per time-step. Step 1 involves the resolution of a coupled problem ((37)-(38) or (41)-(38)) of reduced computational complexity (compared with step 1 of Algorithm 2 or with step 2 of Algorithm 5). This coupled problem can be solved, in a partitioned fashion, with simplified versions of the procedures discussed in $\S 3$. As a matter of fact, the main advantages of Algorithm 6 are its simplicity of implementation 
(specially compared to sophisticated Newton-like methods) and its efficiency compared to the solution procedures presented so far. Obviously, its main limitation is that it assumes the fluid to be solved with a projection-based scheme.

Remark 10 The ideas presented here can be generalized to other fractional step schemes in the fluid. For instance, extensions in the framework of algebraic factorization methods have been reported in [103, 12].

Algorithm 6 is based on the following implicit-explicit time discretization of the coupling conditions (13):

$$
\begin{aligned}
& \left\{\begin{array}{r}
\boldsymbol{\Pi}^{n+\frac{1}{2}} \boldsymbol{n}^{\mathrm{s}}=-J^{n} \widehat{\boldsymbol{\sigma}}\left(\widetilde{\boldsymbol{u}}^{n}, p^{n+1}\right)\left(\boldsymbol{F}^{n}\right)^{-\mathrm{T}} \boldsymbol{n} \text { on } \Sigma, \\
\widehat{\boldsymbol{u}}^{n+1} \cdot \boldsymbol{n}=\partial_{\tau} \boldsymbol{d}^{n+1} \cdot \boldsymbol{n} \text { on } \Sigma,
\end{array}\right. \\
& \left\{\begin{array}{l}
\boldsymbol{d}^{\mathrm{f}, n+1}=\operatorname{Ext}\left(\left.\boldsymbol{d}^{n}\right|_{\Sigma}\right), \quad \widehat{\boldsymbol{w}}^{n+1}=\partial_{\tau} \boldsymbol{d}^{\mathrm{f}, n+1}, \quad \Omega^{\mathrm{f}, n+1}=\left(\boldsymbol{I}_{\Omega^{\mathrm{f}}}+\boldsymbol{d}^{\mathrm{f}, n+1}\right)\left(\Omega^{\mathrm{f}}\right), \\
\widehat{\widehat{\boldsymbol{u}}}^{n+1}=\partial_{\tau} \boldsymbol{d}^{n+1} \quad \text { on } \quad \Sigma .
\end{array}\right.
\end{aligned}
$$

Note that $(13)_{3}$ is not exactly enforced at each time-step and, therefore, Algorithm 6 is not a strongly coupled scheme. Yet, this scheme is also not loosely coupled since $(13)_{3}$ is not treated explicitly (namely, the solid displacement and the fluid pressure are implicitly coupled).

\section{Stability analysis (linear case)}

In [48], the stability of Algorithm 6 has been analyzed in the framework of the following linear model problem, coupling the Stokes equations with a linear elastic solid model:

$$
\begin{aligned}
& \left\{\begin{array}{rll}
\rho^{\mathrm{f}} \partial_{t} \boldsymbol{u}-\operatorname{div} \boldsymbol{\sigma}(\boldsymbol{u}, p)=\mathbf{0} & \text { in } \quad \Omega^{\mathrm{f}}, \\
\operatorname{div} \boldsymbol{u}=0 & \text { in } & \Omega^{\mathrm{f}}, \\
\boldsymbol{u}=\boldsymbol{u}_{\text {in }} & \text { on } & \Gamma^{\text {in }}, \\
\boldsymbol{\sigma}(\boldsymbol{u}, p) \boldsymbol{n}=p_{\text {out }} \boldsymbol{n} & \text { on } \quad \Gamma^{\text {out }},
\end{array}\right. \\
& \left\{\begin{aligned}
\rho^{\mathrm{s}} \partial_{t t} \boldsymbol{d}-\operatorname{div} \boldsymbol{\sigma}(\boldsymbol{d})=\mathbf{0} & \text { in } \quad \Omega^{\mathrm{s}}, \\
\boldsymbol{d}=\mathbf{0} & \text { on } \quad \Gamma^{\mathrm{d}}, \\
\boldsymbol{\sigma}(\boldsymbol{d}) \boldsymbol{n}^{\mathrm{s}}=\mathbf{0} & \text { on } \quad \Gamma^{\mathrm{n}},
\end{aligned}\right. \\
& \left\{\begin{array}{rcc}
\boldsymbol{u}=\partial_{t} \boldsymbol{d} & \text { on } \quad \Sigma, \\
\boldsymbol{\sigma}(\boldsymbol{d}) \boldsymbol{n}^{\mathrm{s}}=-\boldsymbol{\sigma}(\boldsymbol{u}, p) \boldsymbol{n} & \text { on } \quad \Sigma .
\end{array}\right.
\end{aligned}
$$

Though simplified, the linear coupled problem (42)-(44) contains the key features of more complex fluid-structure problems involving an incompressible fluid, as regards the stability of the coupling schemes (see, e.g., [25]).

Since the analysis is carried out in the fully discrete case, we need to introduce some notation for the discretization in space. We define $Q_{h}^{\mathrm{f}}$ as an 
internal continuous Lagrange finite element approximation of $L^{2}\left(\Omega^{\mathrm{f}}\right)$. Similarly, $\boldsymbol{V}_{h}^{\mathrm{f}}$ (resp. $\boldsymbol{V}_{\omega, h}^{\mathrm{f}}$, with $\omega \subset \partial \Omega^{\mathrm{f}}$, and $\boldsymbol{V}_{H}^{\mathrm{s}}$ ) is an internal continuous Lagrange finite element approximation of $\boldsymbol{H}^{1}\left(\Omega^{\mathrm{f}}\right)$ (resp. $\boldsymbol{H}_{\omega}^{1}\left(\Omega^{\mathrm{f}}\right)$ and $\boldsymbol{H}_{\Gamma^{\mathrm{d}}}^{1}\left(\Omega^{\mathrm{s}}\right)$ ). Since the fluid and solid space discretizations do not necessarily match at the interface $\Sigma$, we introduce an interface matching operator $\Pi_{h}: \boldsymbol{V}_{H}^{\mathrm{s}}(\Sigma) \rightarrow \boldsymbol{V}_{h}^{\mathrm{f}}(\Sigma)$, where $\boldsymbol{V}_{H}^{\mathrm{s}}(\Sigma)$ (resp. $\boldsymbol{V}_{h}^{\mathrm{f}}(\Sigma)$ ) stands for the trace finite element space associated to $\boldsymbol{V}_{H}^{\mathrm{s}}\left(\right.$ resp. $\left.\boldsymbol{V}_{h}^{\mathrm{f}}\right)$. The operator $\Pi_{h}$ can be, for instance, the standard Lagrange interpolant (nodal-wise matching) or a projection based operator (see, e.g., $[41,61,7])$.

The fully discretized problem writes as follows: for $n \geq 0$,

1. Implicit step (pressure-structure coupling): find $\left(\boldsymbol{u}_{h}^{n+1}, p_{h}^{n+1}, \boldsymbol{d}_{H}^{n+1}\right) \in$ $\boldsymbol{V}_{h}^{\mathrm{f}} \times Q_{h}^{\mathrm{f}} \times \boldsymbol{V}_{H}^{\mathrm{s}}$ such that

$$
\begin{aligned}
& \left\{\begin{array}{l}
\boldsymbol{u}_{h}^{n+1}=\boldsymbol{u}_{\text {in }}\left(t_{n+1}\right), \quad \text { on } \quad \Gamma^{\mathrm{in}}, \\
\boldsymbol{u}_{h}^{n+1}=\Pi_{h}\left(\partial_{\tau} \boldsymbol{d}_{H}^{n+1}\right), \quad \text { on } \quad \Sigma, \\
\frac{\rho^{\mathrm{f}}}{\tau} \int_{\Omega^{\mathrm{f}}}\left(\boldsymbol{u}_{h}^{n+1}-\widetilde{\boldsymbol{u}}_{h}^{n}\right) \cdot \boldsymbol{v}_{h}^{\mathrm{f}}-\int_{\Omega^{\mathrm{f}}} p_{h}^{n+1} \operatorname{div} \boldsymbol{v}_{h}^{\mathrm{f}}+\int_{\Omega^{\mathrm{f}}} q_{h} \operatorname{div} \boldsymbol{u}_{h}^{n+1} \\
=-\int_{\Gamma^{\text {out }}} p_{\text {out }} \boldsymbol{v}_{h}^{\mathrm{f}} \cdot \boldsymbol{n} \quad \forall\left(\boldsymbol{v}_{h}^{\mathrm{f}}, q_{h}\right) \in \boldsymbol{V}_{\Sigma \cup \Gamma^{\mathrm{in}}, h}^{\mathrm{f}} \times Q_{h}^{\mathrm{f}},
\end{array}\right. \\
& \left\{\begin{array}{l}
\frac{\rho^{\mathrm{s}}}{\tau^{2}} \int_{\Omega^{\mathrm{s}}}\left(\boldsymbol{d}_{H}^{n+1}-2 \boldsymbol{d}_{H}^{n}+\boldsymbol{d}_{H}^{n-1}\right) \cdot \boldsymbol{v}_{H}^{\mathrm{s}}+a^{\mathrm{s}}\left(\boldsymbol{d}_{H}^{n+1}, \boldsymbol{v}_{H}^{\mathrm{s}}\right) \\
=-\left\langle\mathcal{R}_{\mu}\left(\widetilde{\boldsymbol{u}}_{h}^{n}\right), L_{h}\left(\boldsymbol{v}_{H}^{\mathrm{s}}\right)\right\rangle-\left\langle\mathcal{R}_{p}\left(\boldsymbol{u}_{h}^{n+1}, p_{h}^{n+1}\right), L_{h}\left(\left.\boldsymbol{v}_{H}^{\mathrm{s}}\right|_{\Sigma}\right)\right\rangle \quad \forall \boldsymbol{v}_{H}^{\mathrm{s}} \in \boldsymbol{V}_{H}^{\mathrm{s}} ;
\end{array}\right.
\end{aligned}
$$

2. Explicit step (viscous-structure coupling): find $\widetilde{\boldsymbol{u}}_{h}^{n+1} \in \boldsymbol{V}_{h}^{\mathrm{f}}$ such that

$$
\left\{\begin{array}{l}
\widetilde{\boldsymbol{u}}_{h}^{n+1}=\boldsymbol{u}_{\mathrm{in}}\left(t_{n+1}\right), \quad \text { on } \quad \Gamma^{\mathrm{in}}, \\
\widetilde{\boldsymbol{u}}_{h}^{n+1}=\Pi_{h}\left(\partial_{\tau} \boldsymbol{d}_{H}^{n+1}\right), \quad \text { on } \Sigma, \\
\frac{\rho^{\mathrm{f}}}{\tau} \int_{\Omega^{\mathrm{f}}}\left(\widetilde{\boldsymbol{u}}_{h}^{n+1}-\boldsymbol{u}_{h}^{n+1}\right) \cdot \widetilde{\boldsymbol{v}}_{h}^{\mathrm{f}}+2 \mu \int_{\Omega^{\mathrm{f}}} \boldsymbol{\epsilon}\left(\widetilde{\boldsymbol{u}}_{h}^{n+1}\right): \boldsymbol{\epsilon}\left(\widetilde{\boldsymbol{v}}_{h}^{\mathrm{f}}\right) \quad \forall \widetilde{\boldsymbol{v}}_{h}^{\mathrm{f}} \in \boldsymbol{V}_{\Sigma \cup \Gamma^{\mathrm{in}}, h}^{\mathrm{f}} .
\end{array}\right.
$$

Here, $a^{\mathrm{s}}(\cdot, \cdot)$ stands for a general solid stiffness bilinear form and the fluid stress at the interface are given in terms of the (variationally consistent) residuals $\mathcal{R}_{\mu}$ and $\mathcal{R}_{p}$, defined as

$$
\begin{aligned}
&\left\langle\mathcal{R}_{\mu}\left(\widetilde{\boldsymbol{u}}^{n+1}\right), \widetilde{\boldsymbol{v}}^{\mathrm{f}}\right\rangle \stackrel{\text { deff }}{=} \frac{\rho^{\mathrm{f}}}{\tau} \int_{\Omega^{\mathrm{f}}}\left(\widetilde{\boldsymbol{u}}^{n+1}-\boldsymbol{u}^{n+1}\right) \cdot \widetilde{\boldsymbol{v}}^{\mathrm{f}}+2 \mu \int_{\Omega^{\mathrm{f}}} \boldsymbol{\epsilon}\left(\widetilde{\boldsymbol{u}}^{n+1}\right): \boldsymbol{\epsilon}\left(\widetilde{\boldsymbol{v}}^{\mathrm{f}}\right), \\
&\left\langle\mathcal{R}_{p}\left(\boldsymbol{u}^{n+1}, p^{n+1}\right), \boldsymbol{v}^{\mathrm{f}}\right\rangle \stackrel{\text { def }}{=} \frac{\rho^{\mathrm{f}}}{\tau} \int_{\Omega^{\mathrm{f}}}\left(\boldsymbol{u}^{n+1}-\widetilde{\boldsymbol{u}}^{n}\right) \cdot \boldsymbol{v}^{\mathrm{f}}-\int_{\Omega^{\mathrm{f}}} p^{n+1} \operatorname{div} \boldsymbol{v}^{\mathrm{f}},
\end{aligned}
$$

and $L_{h}: \boldsymbol{V}_{H}^{\mathrm{s}}(\Sigma) \rightarrow \boldsymbol{V}_{h}^{\mathrm{f}}$ stands for the standard discrete lifting operator, satisfying $\left.L_{h}\left(\boldsymbol{b}_{H}\right)\right|_{\Sigma}=\Pi_{h}\left(\left.\boldsymbol{b}_{H}\right|_{\Sigma}\right)$ and $\left.L_{h}\left(\boldsymbol{b}_{H}\right)\right|_{\Gamma^{\text {in }} \cup \Gamma^{\text {out }}}=\mathbf{0}$ for all $\boldsymbol{b}_{H} \in \boldsymbol{V}_{H}^{\mathrm{s}}(\Sigma)$. 
Remark 11 Note that, in (45), we impose $\boldsymbol{u}_{h}^{n+1}=\Pi_{h}\left(\partial_{\tau} \boldsymbol{d}_{H}^{n+1}\right)$ on $\Sigma$ (instead of $(37)_{5}$ ) which is also optimal in the framework of finite element approximations (see [62]).

Remark 12 We have considered here a simplified version of the coupling scheme given by Algorithm 6. The fluid domain being fixed, no ALE terms appear in the equations. Moreover, we assumed that the solid equations are discretized in time with a (non-conservative) leap-frog scheme. In spite of that, the main feature of the coupling scheme is preserved: the diffusion step is explicitly coupled with the structure. Without these simplifications, the stability analysis does not seem to be straightforward.

Let

$$
E^{n} \stackrel{\text { def }}{=} \frac{\rho^{\mathrm{f}}}{2}\left\|\boldsymbol{u}_{h}^{n}\right\|_{0, \Omega^{\mathrm{f}}}^{2}+\frac{\rho^{\mathrm{s}}}{2}\left\|\partial_{\tau} \boldsymbol{d}_{H}^{n}\right\|_{0, \Omega^{\mathrm{s}}}^{2}+\frac{1}{2} a^{\mathrm{s}}\left(\boldsymbol{d}_{H}^{n}, \boldsymbol{d}_{H}^{n}\right)
$$

be the discrete energy of the system at time-step $n$. In what follows, the symbol $\lesssim$ indicates an inequality up to a multiplicative constant independent of the discretization and physical parameters.

The following result (from [48]) provides the conditional stability of the coupling scheme (45)-(46).

Theorem 2 Assume that $p_{\text {out }}=0, \boldsymbol{u}_{\text {in }}=\mathbf{0}, \mathcal{R}_{\mu}\left(\widetilde{\boldsymbol{u}}_{h}^{0}\right)=0$ and that the interface matching operator $\Pi_{h}: V_{H}^{\mathrm{s}}(\Sigma) \longrightarrow V_{h}^{\mathrm{f}}(\Sigma)$ is $L^{2}$-stable. Then, under the condition

$$
\left(\rho^{\mathrm{f}} \frac{h}{H^{\alpha}}+2 \frac{\mu \tau}{h H^{\alpha}}\right) \lesssim \rho^{\mathrm{s}}, \quad \text { with } \alpha \stackrel{\text { def }}{=} \begin{cases}0, & \text { if } \overline{\Omega^{\mathrm{s}}}=\Sigma, \\ 1, & \text { if } \overline{\Omega^{\mathrm{s}}} \neq \Sigma,\end{cases}
$$

there holds,

$$
E^{n}+\mu \sum_{m=0}^{n-1} \tau\left\|\boldsymbol{\epsilon}\left(\widetilde{\boldsymbol{u}}_{h}^{m+1}\right)\right\|_{0, \Omega^{\mathrm{f}}}^{2} \lesssim E^{0}
$$

for $n \geq 1$.

Some observations are now in order:

- The assumption on the $L^{2}$-stability of the interface matching operator is satisfied by the standard finite element interpolation operator, for example, whenever the fluid interface triangulation is a sub-triangulation of the solid interface triangulation. This includes, in particular, the case of interface matching meshes. By construction, a mortar based matching operator also fulfills that assumption (see [17]).

- The sufficient condition (47) can be satisfied by reducing the ratios $h / H^{\alpha}$ and $\tau /\left(h H^{\alpha}\right)$. The later might be thought as a CFL-like condition. Note that this is a major advantage compared to the (in)stability condition (5) for the explicit-coupling scheme. 
- In the case $\overline{\Omega^{\mathrm{s}}}=\Sigma$ (thin structure model), i.e., $\alpha=0$, condition (47) becomes independent of the solid mesh size $H$. In particular, we may set $H=h$, and stabilize the scheme by reducing $h$ (and $\tau$ ).

- In the case $\overline{\Omega^{\mathrm{s}}} \neq \Sigma$, i.e., $\alpha=1$, the stability of the scheme can be ensured provided that the fluid mesh size $h$ is small enough compared to the structure mesh size $H$. Numerical simulations performed in 2D and 3D, with $h=H$, showed however that this condition seems to be not necessary, when dealing with physiological parameters.

Remark 13 We refer to [7] for an a priori error analysis of (45)-(46) which ensures an overall $O\left(\tau^{\frac{1}{2}}+h^{k}+H^{m}+h^{l}\right)$ convergence rate in the energy norm. Here, $k, m$ are respectively the polynomial degrees of the fluid and solid discretizations and $l$ depends on the choice of the matching operator $\Pi_{h}$.

\begin{tabular}{|c|c|c|}
\hline COUPLING & ALGORITHM & CPU time \\
\hline \multirow{3}{*}{ Implicit } & FP-Aitken & 24.86 \\
& Inexact-Newton & 6.05 \\
& Newton & 4.77 \\
\hline Semi-Implicit & Newton & 1 \\
\hline
\end{tabular}

Table 1: Elapsed CPU time (dimensionless): straight cylinder, 50 time steps of length $\tau=2 \times 10^{-4} \mathrm{~s}$. From [48].

We conclude this section with a few numerical illustrations, involving the coupling of the ALE Navier-Stokes equations with a non-linear shell model (based on MITC4 shell elements [26]). Table 1 shows a comparison of the elapsed CPU times (dimensionless) obtained in the simulation of a pressure wave propagation in a compliant straight vessel $($ see $[52,59])$. We can notice that the semi-implicit coupling is 4.7 times faster than the best implicit coupling. This performance rises much more when considering a more physiological situation. In Table 2 we have reported the the elapsed CPU times (dimensionless) obtained in the simulation of two cardiac cycles of blood flow in a idealized abdominal aortic aneurysm (see Figure 4 (left)) under physiological conditions (see [106]). The accuracy of the semi-implicit coupling scheme is highlighted in Figure 4 (right), in terms of the outflow rate.

\begin{tabular}{|c|c|}
\hline COUPLING & CPU time \\
\hline Implicit & 9.3 \\
Semi-Implicit & 1.0 \\
\hline
\end{tabular}

Table 2: Elapsed CPU time (dimensionless): blood flow in a idealized abdominal aortic aneurysm, 2 cardiac cycles (1000 time-steps of length $\tau=1.68 \times 10^{-3} \mathrm{~s}$ ). 

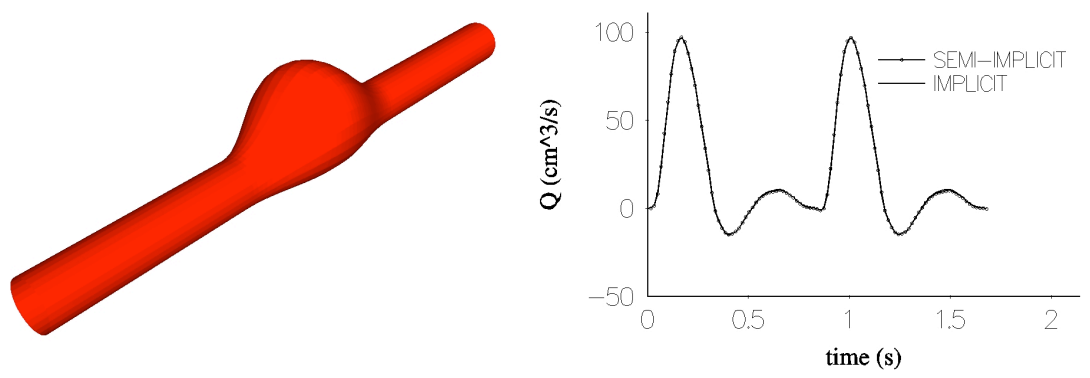

Figure 4: Left: idealized abdominal aortic aneurysm. Right: comparison of implicit and semi-implicit coupling schemes, outflow rate.

\section{Nitsche's based interface treatment}

The coupling schemes described in the previous sections treat the interface condition $(13)_{2}$ as a (strongly imposed) Dirichlet boundary condition in the fluid, and $(13)_{3}$ as a Neumann boundary condition in the solid, respectively. We shall see, in this section, that an appropriate weak treatment of these interface conditions, based on Nitsche's interface method [16, 67], benefits from:

- a specific treatment of the viscous contributions;

- further insights on the instability of explicit coupling (Algorithm 1).

These key features motivated the derivation of a stabilized explicit coupling scheme, in [22], and of a new semi-implicit coupling scheme, in [5], whose stability properties are independent of the added-mass effect. These schemes are presented in $\S 5.1$ and in $\S 5.2$, respectively.

\subsection{Stabilized explicit coupling}

For the sake of simplicity and without loss of generality (see Remark 21 below), in this subsection we limit the presentation to the case of the linear coupled problem (42)-(44). We shall also make use of some of the notations introduced in $\S 4.1$ for the discretization in space.

\section{Space semi-discretization: interface Nitsche's formulation}

Originally, Nitsche's method [95] is a technique for enforcing Dirichlet boundary conditions in a weak sense (instead of being built into the finite element space). The method has recently been generalized to other boundary conditions [75]. The extension of Nitsche's method to the approximation of elliptic and parabolic problems with discontinuous piecewise polynomials is known as discontinuous Galerkin (DG) method (see, e.g., [13, 119, 2, 3]). In [16], Nitsche's method was proposed for the approximation of an interface problem 
arising in the framework of domain decomposition with non-matching grids. Since then, it has been extended to different multi-physics problems (see, e.g., $[24,23]$, and [67] for a review). In the context of fluid-structure interaction, using implicit coupling, some results are given for vibration problems (acoustics) in [68] and for transient fluid-structure interaction problems with moving fluid domains in [69].

The space semi-discrete Nitsche's interface formulation of (42)-(44) proposed in [22] is given as follows: find $\left(\boldsymbol{u}_{h}, p_{h}, \boldsymbol{d}_{h}, \dot{\boldsymbol{d}}_{h}\right) \in \boldsymbol{V}_{h}^{\mathrm{f}} \times Q_{h} \times\left[\boldsymbol{V}_{h}^{\mathrm{s}}\right]^{2}$, with $\boldsymbol{u}_{h}=\boldsymbol{u}_{\text {in }}$ on $\Gamma^{\text {in }}$, such that

$$
\begin{aligned}
& A^{\mathrm{f}}\left(\left(\boldsymbol{u}_{h}, p_{h}\right),\left(\boldsymbol{v}_{h}^{\mathrm{f}}, q_{h}\right)\right)+A^{\mathrm{s}}\left(\left(\boldsymbol{d}_{h}, \dot{\boldsymbol{d}}_{h}\right),\left(\boldsymbol{v}_{h}^{\mathrm{s}}, \boldsymbol{r}_{h}\right)\right) \\
& -\int_{\Sigma} \boldsymbol{\sigma}\left(\boldsymbol{u}_{h}, p_{h}\right) \boldsymbol{n} \cdot\left(\boldsymbol{v}_{h}^{\mathrm{f}}-\boldsymbol{v}_{h}^{\mathrm{s}}\right)-\int_{\Sigma}\left(\boldsymbol{u}_{h}-\partial_{t} \boldsymbol{d}_{h}\right) \cdot \boldsymbol{\sigma}\left(\boldsymbol{v}_{h}^{\mathrm{f}},-q_{h}\right) \boldsymbol{n} \\
& \quad+\frac{\gamma \mu}{h} \int_{\Sigma}\left(\boldsymbol{u}_{h}-\partial_{t} \boldsymbol{d}_{h}\right) \cdot\left(\boldsymbol{v}_{h}^{\mathrm{f}}-\boldsymbol{v}_{h}^{\mathrm{s}}\right)=-\int_{\Gamma_{\text {out }}} p_{\text {out }} \boldsymbol{v}_{h}^{\mathrm{f}} \cdot \boldsymbol{n}
\end{aligned}
$$

for all $\left(\boldsymbol{v}_{h}^{\mathrm{f}}, q_{h}, \boldsymbol{v}_{h}^{\mathrm{s}}, \boldsymbol{r}_{h}\right) \in \boldsymbol{V}_{\Gamma^{\mathrm{in}}, h}^{\mathrm{f}} \times Q_{h} \times\left[\boldsymbol{V}_{h}^{\mathrm{s}}\right]^{2}$. Here, $\gamma>0$ is a dimensionless penalty parameter (specified below), and the fluid and solid volume contributions are given by

$$
\begin{aligned}
& A^{\mathrm{f}}\left(\left(\boldsymbol{u}_{h}, p_{h}\right),\left(\boldsymbol{v}_{h}^{\mathrm{f}}, q_{h}\right)\right) \stackrel{\text { def }}{=} \rho^{\mathrm{f}} \int_{\Omega^{\mathrm{f}}} \partial_{t} \boldsymbol{u}_{h} \cdot \boldsymbol{v}_{h}^{\mathrm{f}}+2 \mu \int_{\Omega^{\mathrm{f}}} \boldsymbol{\epsilon}\left(\boldsymbol{u}_{h}\right): \boldsymbol{\epsilon}\left(\boldsymbol{v}_{h}^{\mathrm{f}}\right)-\int_{\Omega^{\mathrm{f}}} p_{h} \operatorname{div} \boldsymbol{v}_{h}^{\mathrm{f}} \\
&+\int_{\Omega^{\mathrm{f}}} q_{h} \operatorname{div} \boldsymbol{u}_{h}, \\
& A^{\mathrm{s}}\left(\left(\boldsymbol{d}_{h}, \dot{\boldsymbol{d}}_{h}\right),\left(\boldsymbol{v}_{h}^{\mathrm{s}}, \boldsymbol{r}_{h}\right)\right) \stackrel{\text { def }}{=} \rho^{\mathrm{s}} \int_{\Omega^{\mathrm{s}}} \partial_{t} \dot{\boldsymbol{d}}_{h} \cdot \boldsymbol{v}_{h}^{\mathrm{s}}+a^{\mathrm{s}}\left(\boldsymbol{d}_{h}, \boldsymbol{v}_{h}^{\mathrm{s}}\right)+\rho^{\mathrm{s}} \int_{\Omega^{\mathrm{f}}}\left(\dot{\boldsymbol{d}}_{h}-\partial_{t} \boldsymbol{d}_{h}\right) \cdot \boldsymbol{r}_{h} .
\end{aligned}
$$

Remark 14 Note that, in (48), $\boldsymbol{u}_{h}$ and $\partial_{t} \boldsymbol{d}_{h}$ (and $\boldsymbol{v}_{h}^{\mathrm{f}}$ and $\boldsymbol{v}_{h}^{\mathrm{s}}$ ) do not necessarily match at the interface (interface $D G$ formulation). The interface integrals involving the fluid stress $\boldsymbol{\sigma}\left(\boldsymbol{u}_{h}, p_{h}\right) \boldsymbol{n}$ are computed face-wise, as broken integrals.

By taking alternatively $\left(\boldsymbol{v}_{h}^{\mathrm{f}}, q_{h}\right)=\mathbf{0}$ and $\boldsymbol{v}_{h}^{\mathrm{s}}=\boldsymbol{r}_{h}=\mathbf{0}$ in (48), this monolithic problem can be reformulated (in a partitioned fashion) in terms of two interface coupled problems:

- Solid subproblem: find $\left(\boldsymbol{d}_{h}, \dot{\boldsymbol{d}}_{h}\right) \in\left[\boldsymbol{V}_{h}^{\mathrm{s}}\right]^{2}$ such that

$$
\begin{aligned}
A^{\mathrm{s}}\left(\left(\boldsymbol{d}_{h}, \dot{\boldsymbol{d}}_{h}\right),\left(\boldsymbol{v}_{h}^{\mathrm{s}}, \boldsymbol{r}_{h}\right)\right)+\frac{\gamma \mu}{h} & \int_{\Sigma} \partial_{t} \boldsymbol{d}_{h} \cdot \boldsymbol{v}_{h}^{\mathrm{s}} \\
& =\frac{\gamma \mu}{h} \int_{\Sigma} \boldsymbol{u}_{h} \cdot \boldsymbol{v}_{h}^{\mathrm{s}}-\int_{\Sigma} \boldsymbol{\sigma}\left(\boldsymbol{u}_{h}, p_{h}\right) \boldsymbol{n} \cdot \boldsymbol{v}_{h}^{\mathrm{s}}
\end{aligned}
$$

for all $\left(\boldsymbol{v}_{h}^{\mathrm{s}}, \boldsymbol{r}_{h}\right) \in\left[\boldsymbol{V}_{h}^{\mathrm{s}}\right]^{2}$; 
- Fluid subproblem: find $\left(\boldsymbol{u}_{h}, p_{h}\right) \in \boldsymbol{V}_{h}^{\mathrm{f}} \times Q_{h}$, with $\boldsymbol{u}_{h}=\boldsymbol{u}_{\text {in }}$ on $\Gamma^{\text {in }}$, such that

$$
\begin{aligned}
& A^{\mathrm{f}}\left(\left(\boldsymbol{u}_{h}, p_{h}\right),\left(\boldsymbol{v}_{h}^{\mathrm{f}}, q_{h}\right)\right)-\int_{\Sigma} \boldsymbol{\sigma}\left(\boldsymbol{u}_{h}, p_{h}\right) \boldsymbol{n} \cdot \boldsymbol{v}_{h}^{\mathrm{f}} \\
& -\int_{\Sigma} \boldsymbol{u}_{h} \cdot \boldsymbol{\sigma}\left(\boldsymbol{v}_{h}^{\mathrm{f}},-q_{h}\right) \boldsymbol{n}+\frac{\gamma \mu}{h} \int_{\Sigma} \boldsymbol{u}_{h} \cdot \boldsymbol{v}_{h}^{\mathrm{f}}=-\int_{\Sigma} \partial_{t} \boldsymbol{d}_{h} \cdot \boldsymbol{\sigma}\left(\boldsymbol{v}_{h}^{\mathrm{f}},-q_{h}\right) \boldsymbol{n} \\
& \quad+\frac{\gamma \mu}{h} \int_{\Sigma} \partial_{t} \boldsymbol{d}_{h} \cdot \boldsymbol{v}_{h}-\int_{\Gamma_{\text {out }}} p_{\text {out }} \boldsymbol{v}_{h}^{\mathrm{f}} \cdot \boldsymbol{n}
\end{aligned}
$$

for all $\left(\boldsymbol{v}_{h}, q_{h}\right) \in \boldsymbol{V}_{\Gamma^{\mathrm{in}}, h}^{\mathrm{f}} \times Q_{h}$.

\section{Time semi-discretization: implicit and explicit coupling}

As in $\S 1$, we consider a first order backward difference discretization in the fluid and a mid-point rule for the structure. The fully discrete fluid and solid volume terms at time-step $n$ are then given by

$$
\begin{aligned}
& A_{\tau}^{\mathrm{f}}\left(\left(\boldsymbol{u}_{h}^{n+1}, p_{h}^{n+1}\right),\left(\boldsymbol{v}_{h}^{\mathrm{f}}, q_{h}\right)\right) \stackrel{\text { def }}{=} \rho^{\mathrm{f}} \int_{\Omega^{\mathrm{f}}} \partial_{\tau} \boldsymbol{u}_{h}^{n+1} \cdot \boldsymbol{v}_{h}^{\mathrm{f}}+2 \mu \int_{\Omega^{\mathrm{f}}} \boldsymbol{\epsilon}\left(\boldsymbol{u}_{h}^{n+1}\right): \boldsymbol{\epsilon}\left(\boldsymbol{v}_{h}^{\mathrm{f}}\right) \\
&-\int_{\Omega^{\mathrm{f}}} p_{h}^{n+1} \operatorname{div} \boldsymbol{v}_{h}^{\mathrm{f}}+\int_{\Omega^{\mathrm{f}}} q_{h} \operatorname{div} \boldsymbol{u}_{h}^{n+1}, \\
& A_{\tau}^{\mathrm{s}}\left(\left(\boldsymbol{d}_{h}^{n+1}, \dot{\boldsymbol{d}}_{h}^{n+1}\right),\left(\boldsymbol{v}_{h}^{\mathrm{s}}, \boldsymbol{r}_{h}\right)\right) \stackrel{\text { def }}{=} \rho^{\mathrm{s}} \int_{\Omega^{\mathrm{s}}} \partial_{\tau} \dot{\boldsymbol{d}}_{h}^{n+1} \cdot \boldsymbol{v}_{h}^{\mathrm{s}}+a^{\mathrm{s}}\left(\boldsymbol{d}_{h}^{n+\frac{1}{2}}, \boldsymbol{v}_{h}^{\mathrm{s}}\right) \\
&+\rho^{\mathrm{s}} \int_{\Omega^{\mathrm{s}}}\left(\dot{\boldsymbol{d}}_{h}^{n+\frac{1}{2}}-\partial_{\tau} \boldsymbol{d}_{h}^{n+1}\right) \cdot \boldsymbol{r}_{h}
\end{aligned}
$$

and (49)-(50) can be discretized in time as follows: for $n \geq 0$,

- Solid subproblem: find $\left(\boldsymbol{d}_{h}^{n+1}, \dot{\boldsymbol{d}}_{h}^{n+1}\right) \in\left[\boldsymbol{V}_{h}^{\mathrm{s}}\right]^{2}$ such that

$$
\begin{aligned}
A_{\tau}^{\mathrm{s}}\left(\left(\boldsymbol{d}_{h}^{n+1}, \dot{\boldsymbol{d}}_{h}^{n+1}\right),\left(\boldsymbol{v}_{h}^{\mathrm{s}}, \boldsymbol{r}_{h}\right)\right) & +\frac{\gamma \mu}{h} \int_{\Sigma} \partial_{\tau} \boldsymbol{d}_{h}^{n+1} \cdot \boldsymbol{v}_{h}^{\mathrm{s}} \\
& =\frac{\gamma \mu}{h} \int_{\Sigma} \boldsymbol{u}_{h}^{\star} \cdot \boldsymbol{v}_{h}^{\mathrm{s}}-\int_{\Sigma} \boldsymbol{\sigma}\left(\boldsymbol{u}_{h}^{\star}, p_{h}^{\star}\right) \boldsymbol{n} \cdot \boldsymbol{v}_{h}^{\mathrm{s}}
\end{aligned}
$$

for all $\left(\boldsymbol{v}_{h}^{\mathrm{s}}, \boldsymbol{r}_{h}\right) \in\left[\boldsymbol{V}_{h}^{\mathrm{s}}\right]^{2}$;

- Fluid subproblem: find $\left(\boldsymbol{u}_{h}^{n+1}, p_{h}^{n+1}\right) \in \boldsymbol{V}_{h}^{\mathrm{f}} \times Q_{h}$, with $\boldsymbol{u}_{h}^{n+1}=\boldsymbol{u}_{\text {in }}\left(t_{n+1}\right)$ on $\Gamma^{\text {in }}$, such that

$$
\begin{aligned}
A_{\tau}^{\mathrm{f}}\left(\left(\boldsymbol{u}_{h}^{n+1}, p_{h}^{+1}\right),\left(\boldsymbol{v}_{h}^{\mathrm{f}}, q_{h}\right)\right) & -\int_{\Sigma} \boldsymbol{\sigma}\left(\boldsymbol{u}_{h}^{\star}, p_{h}^{\star}\right) \boldsymbol{n} \cdot \boldsymbol{v}_{h}^{\mathrm{f}} \\
-\int_{\Sigma} \boldsymbol{u}_{h}^{n+1} \cdot \boldsymbol{\sigma}\left(\boldsymbol{v}_{h}^{\mathrm{f}},-q_{h}\right) \boldsymbol{n}+ & \frac{\gamma \mu}{h} \int_{\Sigma} \boldsymbol{u}_{h}^{n+1} \cdot \boldsymbol{v}_{h}^{\mathrm{f}}=-\int_{\Sigma} \partial_{\tau} \boldsymbol{d}_{h}^{n+1} \cdot \boldsymbol{\sigma}\left(\boldsymbol{v}_{h}^{\mathrm{f}},-q_{h}\right) \boldsymbol{n} \\
& +\frac{\gamma \mu}{h} \int_{\Sigma} \partial_{\tau} \boldsymbol{d}_{h}^{n+1} \cdot \boldsymbol{v}_{h}^{\mathrm{f}}-\int_{\Gamma_{\text {out }}} p_{\text {out }} \boldsymbol{v}_{h}^{\mathrm{f}} \cdot \boldsymbol{n}
\end{aligned}
$$

for all $\left(\boldsymbol{v}_{h}^{\mathrm{f}}, q_{h}\right) \in \boldsymbol{V}_{\Gamma^{\mathrm{in}}, h}^{\mathrm{f}} \times Q_{h}$. 
If $\left(\boldsymbol{u}_{h}^{\star}, p_{h}^{\star}\right)=\left(\boldsymbol{u}_{h}^{n+1}, p_{h}^{n+1}\right)$, the scheme (51)-(52) corresponds to an implicit coupling scheme. On the contrary, for $\left(\boldsymbol{u}_{h}^{\star}, p_{h}^{\star}\right)=\left(\boldsymbol{u}_{h}^{n}, p_{h}^{n}\right)$ the coupling scheme is explicit (i.e., loosely coupled).

Let $E^{n}$ denote the total discrete energy of the system at the time level $n$, defined by

$$
E^{n} \stackrel{\text { def }}{=} \frac{\rho_{\mathrm{f}}}{2}\left\|\boldsymbol{u}_{h}^{n}\right\|_{0, \Omega_{\mathrm{f}}}^{2}+\frac{\rho_{\mathrm{s}}}{2}\left\|\dot{\boldsymbol{d}}_{h}^{n}\right\|_{0, \Omega_{\mathrm{s}}}^{2}+\frac{1}{2} a^{\mathrm{s}}\left(\boldsymbol{d}_{h}^{n}, \boldsymbol{d}_{h}^{n}\right) .
$$

The next result (from [22]) summarizes the energy based stability of the coupling schemes given by (51)-(52).

Theorem 3 Assume that the fluid-structure system is isolated (i.e., $\boldsymbol{u}_{\mathrm{in}}=\mathbf{0}$ and $\left.p_{\text {out }}=0\right)$ and let $\left(\boldsymbol{u}_{h}^{n+1}, p_{h}^{n+1}, \boldsymbol{d}_{h}^{n+1}, \dot{\boldsymbol{d}}_{h}^{n+1}\right)$ be given by (51)-(52).

- Implicit coupling: For $\left(\boldsymbol{u}_{h}^{\star}, p_{h}^{\star}\right)=\left(\boldsymbol{u}_{h}^{n+1}, p_{h}^{n+1}\right), \gamma \geq 16 C_{\mathrm{TI}}$ and $n \geq 1$, there holds

$$
E^{n}+\mu \sum_{m=0}^{n-1} \tau\left\|\boldsymbol{\epsilon}\left(\boldsymbol{u}_{h}^{m+1}\right)\right\|_{0, \Omega_{\mathrm{f}}}^{2}+\frac{\gamma \mu}{h} \sum_{m=0}^{n-1} \tau\left\|\boldsymbol{u}_{h}^{m+1}-\partial_{\tau} \boldsymbol{d}_{h}^{m+1}\right\|_{0, \Sigma}^{2} \lesssim E^{0} ;
$$

- Explicit coupling: For $\left(\boldsymbol{u}_{h}^{\star}, p_{h}^{\star}\right)=\left(\boldsymbol{u}_{h}^{n}, p_{h}^{n}\right), \gamma \geq 256 C_{\text {TI }}$ and $n \geq 1$, there holds

$$
\begin{array}{r}
E^{n}+\mu \sum_{m=0}^{n-1} \tau\left\|\boldsymbol{\epsilon}\left(\boldsymbol{u}_{h}^{m+1}\right)\right\|_{0, \Omega_{\mathrm{f}}}^{2}+\frac{\gamma \mu}{h} \sum_{m=0}^{n-1} \tau\left\|\boldsymbol{u}_{h}^{m+1}-\partial_{\tau} \boldsymbol{d}_{h}^{m+1}\right\|_{0, \Sigma}^{2} \\
+\frac{\gamma \mu \tau}{h}\left\|\boldsymbol{u}_{h}^{n}\right\|_{0, \Sigma}^{2} \lesssim E^{0}+\frac{\gamma \mu \tau}{h}\left\|\boldsymbol{u}_{h}^{0}\right\|_{0, \Sigma}^{2}+\mu\left\|\boldsymbol{\epsilon}\left(\boldsymbol{u}_{h}^{0}\right)\right\|_{0, \Omega_{\mathrm{f}}}^{2} \\
+\frac{h}{\gamma \mu} \sum_{m=0}^{n-1} \tau\left\|p_{h}^{m+1}-p_{h}^{m}\right\|_{0, \Sigma}^{2}
\end{array}
$$

As expected implicit coupling is unconditionally stable. As regards explicit coupling, Theorem 3 shows that the Nitsche interface penalty and the viscous dissipation control the artificial interface viscous perturbation, generated by the explicit treatment of the coupling. Unfortunately, the artificial interface pressure contribution cannot be directly controlled by the discrete energy of the system $E^{n}$, since we not have control on the time pressure fluctuations at the interface. Somehow this illustrates the already mentioned infamous numerical instability featured by the explicit coupling scheme, when dealing with incompressible fluids (see $\S 1$ and $[25,54]$ ). Yet, the energy estimate (53) suggests that the scheme can be stabilized by the addition of perturbations giving enough control on the time pressure fluctuations at the interface.

Remark 15 The consistency term $-\int_{\Sigma} \boldsymbol{\sigma}\left(\boldsymbol{u}_{h}^{n}, p_{h}^{n}\right) \boldsymbol{n} \cdot \boldsymbol{v}_{h}$ in (52) could also be evaluated at time level $n+1$, as originally proposed in [21]. However, in this case the artificial interface viscous perturbation can not be controlled by the viscous dissipation and the Nitsche's penalty term. 
Remark 16 The energy estimate (53) still remains valid if we neglect the viscous contribution of the (symmetrizing) term $-\int_{\Sigma}\left(\boldsymbol{u}_{h}^{n+1}-\partial_{\tau} \boldsymbol{d}_{h}^{n+1}\right)$. $\boldsymbol{\sigma}\left(\boldsymbol{v}_{h}^{\mathrm{f}},-q_{h}\right) \boldsymbol{n}$ in $(52)$, giving the consistent term $\int_{\Sigma} q_{h}\left(\boldsymbol{u}_{h}^{n+1}-\partial_{\tau} \boldsymbol{d}_{h}^{n+1}\right) \cdot \boldsymbol{n}$. As a result, the explicit coupling scheme (and the mentioned variant) can be formally viewed as a space discrete counterpart of the following Robin-Robin based explicit treatment of (44):

$$
\left.\begin{array}{c}
\boldsymbol{\sigma}\left(\boldsymbol{d}^{n+\frac{1}{2}}\right) \boldsymbol{n}^{\mathrm{s}}+\frac{\gamma \mu}{h} \partial_{\tau} \boldsymbol{d}^{n+1}=\frac{\gamma \mu}{h} \boldsymbol{u}^{n}-\boldsymbol{\sigma}\left(\boldsymbol{u}^{n}, p^{n}\right) \boldsymbol{n} \\
\boldsymbol{\sigma}\left(\boldsymbol{u}^{n+1}, p^{n+1}\right) \boldsymbol{n}+\frac{\gamma \mu}{h} \boldsymbol{u}^{n+1}=\frac{\gamma \mu}{h} \partial_{\tau} \boldsymbol{d}^{n+1}+\boldsymbol{\sigma}\left(\boldsymbol{u}^{n}, p^{n}\right) \boldsymbol{n}
\end{array}\right\} \text { on } \Sigma .
$$

We will come back to this observation in $§ 5.2$.

Remark 17 Note that the scaling $\gamma \mu / h$ of the so-called Robin parameter in (54) is provided by the Nitsche interface method. This choice differs from the RobinRobin scaling proposed in [9], based on simplified models and which aims at accelerating partitioned iterative solution methods within a fully implicit coupling framework. At last, it is worth mentioning that (54) also differs from recent Robin-Robin procedures proposed for time-dependent problems, in the framework of waveform relaxation methods (see, e.g., [57]).

\section{Stabilized explicit coupling}

The spurious oscillations of the fluid pressure at the interface, arising in the energy estimate (53), can be controlled by the following weakly consistent penalty term:

$$
S\left(p_{h}^{n+1}, q_{h}\right) \stackrel{\text { def }}{=} \frac{\gamma_{0} h}{\gamma \mu} \int_{\Sigma}\left(p_{h}^{n+1}-p_{h}^{n}\right) q_{h},
$$

with $\gamma_{0}>0$ a (dimensionless) parameter to be chosen sufficiently large (see Theorem 4). Hence, in [22], we propose to add (55) to the fluid subproblem (52). The resulting stabilized explicit coupling scheme is given in Algorithm 7.

The next theorem (from [22]) provides an energy estimate for the stabilized explicit coupling scheme (Algorithm 7).

Theorem 4 Assume that the fluid-structure system is isolated (i.e., $\boldsymbol{u}_{\mathrm{in}}=\mathbf{0}$ and $p_{\text {out }}=0$ ) and let $\left(\boldsymbol{u}_{h}^{n+1}, p_{h}^{n+1}, \boldsymbol{d}_{h}^{n+1}, \dot{\boldsymbol{d}}_{h}^{n+1}\right)$ be given by Algorithm \%. For $\gamma \geq 256 C_{\mathrm{TI}}, \gamma_{0} \geq 8$ and $n \geq 1$, the following energy estimate holds

$$
\begin{gathered}
E^{n}+\mu \sum_{m=0}^{n-1} \tau\left\|\boldsymbol{\epsilon}\left(\boldsymbol{u}_{h}^{m+1}\right)\right\|_{0, \Omega_{\mathrm{f}}}^{2}+\frac{\gamma \mu}{h} \sum_{m=0}^{n-1} \tau\left\|\boldsymbol{u}_{h}^{m+1}-\partial_{\tau} \boldsymbol{d}_{h}^{m+1}\right\|_{0, \Sigma}^{2}+\frac{\gamma \mu \tau}{h}\left\|\boldsymbol{u}_{h}^{n}\right\|_{0, \Sigma}^{2} \\
+\frac{\gamma_{0} h \tau}{\gamma \mu}\left\|p_{h}^{n}\right\|_{0, \Sigma}^{2} \lesssim E^{0}+\frac{\gamma \mu \tau}{h}\left\|\boldsymbol{u}_{h}^{0}\right\|_{0, \Sigma}^{2}+\mu\left\|\boldsymbol{\epsilon}\left(\boldsymbol{u}_{h}^{0}\right)\right\|_{0, \Omega^{\mathrm{f}}}^{2}+\frac{\gamma_{0} h \tau}{\gamma \mu}\left\|p_{h}^{0}\right\|_{0, \Sigma}^{2}
\end{gathered}
$$

Therefore, Algorithm 7 is energy stable under the (hyperbolic-CFL like) condition

$$
\tau=\mathcal{O}(h)
$$




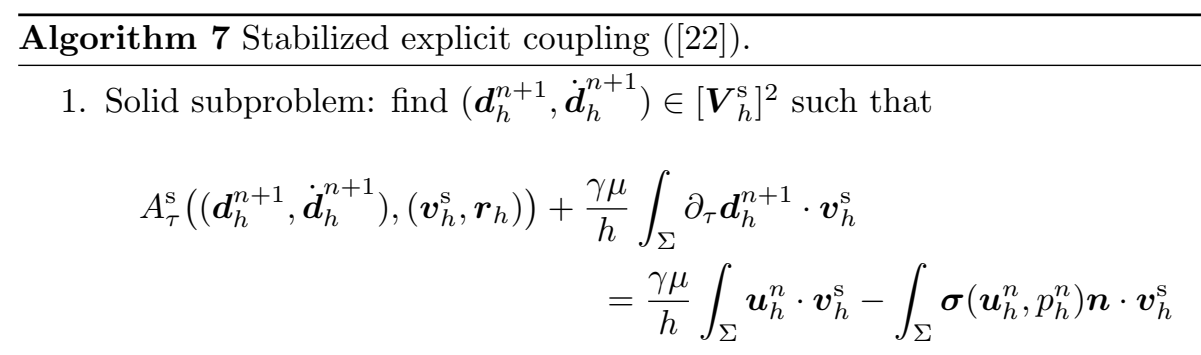

for all $\left(\boldsymbol{v}_{h}^{\mathrm{s}}, \boldsymbol{r}_{h}\right) \in\left[\boldsymbol{V}_{h}^{\mathrm{s}}\right]^{2}$;

2. Fluid subproblem: find $\left(\boldsymbol{u}_{h}^{n+1}, p_{h}^{n+1}\right) \in \boldsymbol{V}_{h}^{\mathrm{f}} \times Q_{h}$, with $\boldsymbol{u}_{h}^{n+1}=\boldsymbol{u}_{\text {in }}\left(t_{n+1}\right)$ on $\Gamma^{\text {in }}$, such that

$$
\begin{aligned}
& A_{\tau}^{\mathrm{f}}\left(\left(\boldsymbol{u}_{h}^{n+1}, p_{h}^{+1}\right),\left(\boldsymbol{v}_{h}^{\mathrm{f}}, q_{h}\right)\right)-\int_{\Sigma} \boldsymbol{u}_{h}^{n+1} \cdot \boldsymbol{\sigma}\left(\boldsymbol{v}_{h}^{\mathrm{f}},-q_{h}\right) \boldsymbol{n} \\
& \quad+\frac{\gamma \mu}{h} \int_{\Sigma} \boldsymbol{u}_{h}^{n+1} \cdot \boldsymbol{v}_{h}^{\mathrm{f}}+S\left(p_{h}^{n+1}, q_{h}\right)=\int_{\Sigma} \boldsymbol{\sigma}\left(\boldsymbol{u}_{h}^{n}, p_{h}^{n}\right) \boldsymbol{n} \cdot \boldsymbol{v}_{h}^{\mathrm{f}} \\
& \quad-\int_{\Sigma} \partial_{\tau} \boldsymbol{d}_{h}^{n+1} \cdot \boldsymbol{\sigma}\left(\boldsymbol{v}_{h}^{\mathrm{f}},-q_{h}\right) \boldsymbol{n}+\frac{\gamma \mu}{h} \int_{\Sigma} \partial_{\tau} \boldsymbol{d}_{h}^{n+1} \cdot \boldsymbol{v}_{h}^{\mathrm{f}}-\int_{\Gamma \text { out }} p_{\text {out }} \boldsymbol{v}_{h}^{\mathrm{f}} \cdot \boldsymbol{n}
\end{aligned}
$$

for all $\left(\boldsymbol{v}_{h}^{\mathrm{f}}, q_{h}\right) \in \boldsymbol{V}_{\Gamma^{\mathrm{in}}, h}^{\mathrm{f}} \times Q_{h}$.

3. Go to next time-step.

Two observations are now in order.

- According to Theorem 4, the stability of Algorithm 7 is independent of the added-mass effect: the fluid-solid density ratio $\rho_{\mathrm{f}} / \rho_{\mathrm{s}}$ and the length of the domain do not come into play. This observation is confirmed by the numerical results reported in Figure 5, corresponding to the simulation of the pressure wave propagation in a two-dimensional straight channel. The numerical solution remains stable irrespectively of the amount of added-mass effect. Note that this is a major advantage compared to standard explicit-coupling schemes, whose (in)stability is dictated by these quantities, irrespectively of the discretization parameters (see the discussion in $\S 1$ and $[25,54])$.

- The proof of Theorem 4 is based, exclusively, on the dissipation due to the Nitsche coupling and the time pressure penalization term. As a result, the stability result is independent of the dissipative features of the fluid and solid time discretization schemes. This is a significant progress with respect to the stability result stated in Theorem 2, for the semiimplicit coupling scheme (45)-(46), whose proof depends on the dissipative properties of the solid time discretization scheme (see Remark 12). On 
the other hand, as regards the fluid time-discretization, one could use, for instance, a neutrally stable second order scheme.

Remark 18 The discrete continuity equation in step 2 of Algorithm 7 is given by

$$
\frac{\gamma_{0} h \tau}{\gamma \mu} \int_{\Sigma} \partial_{\tau} p_{h}^{n+1} q_{h}+\int_{\Omega^{\mathrm{f}}} q_{h} \operatorname{div} \boldsymbol{u}_{h}^{n+1}=\int_{\Sigma}\left(\boldsymbol{u}_{h}^{n+1}-\partial_{\tau} \boldsymbol{d}_{h}^{n+1}\right) \cdot \boldsymbol{n} q_{h} .
$$

The right hand-side being a consistent term, we can interpret the stabilization term (55) as a weakly-consistent interface artificial compressibility. The proposed approach has therefore clear connexions with the already mentioned interface artificial compressibility methods [104, 73, 33] for solving implicit coupling in a partitioned fashion. In these iterative procedures, the compressibility term vanishes at convergence and the artificial compressibility parameter is chosen so as to optimize efficiency and not for consistency or stability purposes.

Remark 19 From Remarks 16 and 18, we can conclude that the main ingredients in the stability of Algorithm 7 are:

- the Robin-Robin based explicit treatment of the interface coupling conditions (54);

- the interface artificial compressibility perturbation of the continuity equation (58).

Remark 20 In the framework of a simplified structural behavior, given in terms of a $(d-1)$-dimensional model (e.g., plates, membranes, shells or inertial-algebraic models), alternative explicit coupling schemes have recently been reported in [65, 64, 44] (see also [97, \$4.1.1]). Since in this case $\Sigma=\overline{\Omega^{\mathrm{s}}}$, the coupling condition (44) can be embedded into the fluid equations (as a Robin boundary condition) and, hence, treated implicitly through a specific (inertial/elastic) time-splitting of the solid equation. A generalization of these schemes to the case of thick structures can be found in [50].

In [22], a formal error estimate for the stabilized explicit coupling scheme is obtained from the energy estimate provided by Theorem 4 . Let $\boldsymbol{\theta}_{h}^{n} \stackrel{\text { def }}{=}$ $\boldsymbol{u}_{h}^{n}-\pi_{h} \boldsymbol{u}\left(t_{n}\right), y_{h}^{n} \stackrel{\text { def }}{=} p_{h}^{n}-\pi_{h} p\left(t_{n}\right), \boldsymbol{\xi}_{h}^{n} \stackrel{\text { def }}{=} \boldsymbol{d}_{h}^{n}-\pi_{h} \boldsymbol{d}\left(t_{n}\right)$ and $\dot{\boldsymbol{\xi}}_{h}^{n} \stackrel{\text { def }}{=} \dot{\boldsymbol{d}}_{h}^{n}-\pi_{h} \dot{\boldsymbol{d}}\left(t_{n}\right)$ be the discrete errors, where $\pi_{h}$ denotes a suitable interpolation operator. We can derive the following error estimate

$$
\mathcal{E}^{n} \lesssim \mathcal{E}^{0}+r_{1}+r_{2}
$$

where

$$
\begin{aligned}
\mathcal{E}^{n} \stackrel{\text { def }}{=} & \left(\frac{\rho^{\mathrm{f}}}{2}\left\|\boldsymbol{\theta}_{h}^{n}\right\|_{0, \Omega^{\mathrm{f}}}^{2}+\frac{\rho^{\mathrm{s}}}{2}\left\|\dot{\boldsymbol{\xi}}_{h}^{n}\right\|_{0, \Omega^{\mathrm{s}}}^{2}+\frac{1}{2} a^{\mathrm{s}}\left(\boldsymbol{\xi}_{h}^{n}, \boldsymbol{\xi}_{h}^{n}\right)+\mu \sum_{m=0}^{n-1} \tau\left\|\boldsymbol{\epsilon}\left(\boldsymbol{\theta}_{h}^{m+1}\right)\right\|_{0, \Omega^{\mathrm{f}}}^{2}\right. \\
& \left.+\frac{\gamma \mu \tau}{h}\left\|\boldsymbol{\theta}_{h}^{n}\right\|_{0, \Sigma}^{2}+\frac{\gamma_{0} h \tau}{\gamma \mu}\left\|y_{h}^{n}\right\|_{0, \Sigma}^{2}+\frac{\gamma \mu \tau}{h} \sum_{m=0}^{n-1}\left\|\boldsymbol{\theta}_{h}^{m+1}-\partial_{\tau} \boldsymbol{\xi}_{h}^{m+1}\right\|_{0, \Sigma}^{2}\right)^{\frac{1}{2}} .
\end{aligned}
$$



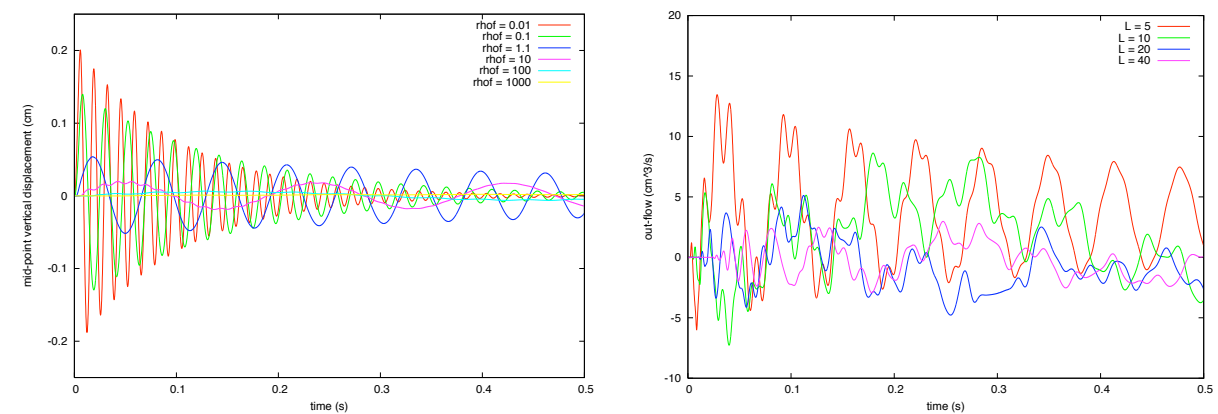

Figure 5: Stabilized explicit coupling (Algorithm 7). Left: interface mid-point $y$-displacement for different values of the fluid-solid density ratio $\rho_{\mathrm{f}} / \rho_{\mathrm{s}}$. Right: out-flow rate for different values of the vessel length $L$. From [22].

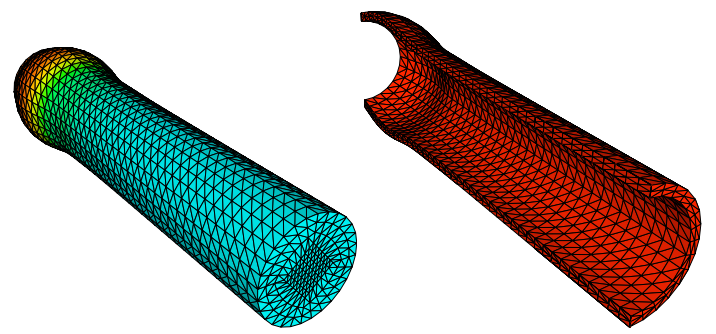

(a) $t=0.0025 \mathrm{~s}$

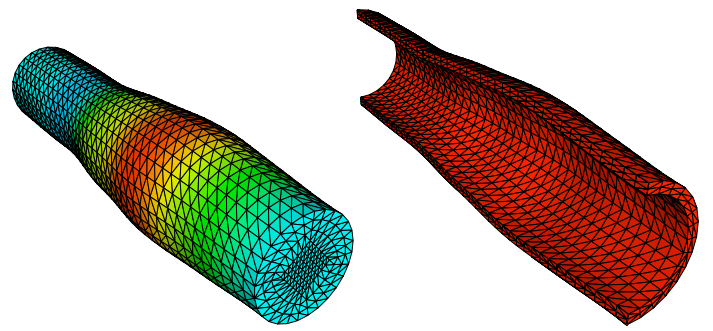

(b) $t=0.01 \mathrm{~s}$

Figure 6: Stabilized explicit coupling without correction: snapshots of the pressure and solid deformation (exaggerated) at two time instants. From [22].

The terms on the right hand side of (59) consists of $\mathcal{E}^{0}$, which measures the error in the initial data, $r_{1}$, which consists of the terms related to the consistency of the fluid and solid discretizations, and $r_{2}$, which contains the explicit coupling consistency error and the weak-consistency introduced by the time penalty 
Algorithm 8 Stabilized explicit coupling with $K \geq 0$ corrections ([22]).

1. Set $\boldsymbol{u}_{h}^{n+1,0} \stackrel{\text { def }}{=} \boldsymbol{u}_{h}^{n}$ and $p_{h}^{n+1,0} \stackrel{\text { def }}{=} p_{h}^{n}$;

2. Correction loop: for $k=0, \ldots, K$ solve

a) Solid subproblem: find $\left(\boldsymbol{d}_{h}^{n+1, k+1}, \dot{\boldsymbol{d}}_{h}^{n+1, k+1}\right) \in\left[\boldsymbol{V}_{h}^{\mathrm{s}}\right]^{2}$ such that

$$
\begin{aligned}
A_{\tau}^{\mathrm{s}}\left(\left(\boldsymbol{d}_{h}^{n+1, k+1}, \dot{\boldsymbol{d}}_{h}^{n+1, k+1}\right),\left(\boldsymbol{v}_{h}^{\mathrm{s}}, \boldsymbol{r}_{h}\right)\right)+\frac{\gamma \mu}{h} \int_{\Sigma} \partial_{\tau} \boldsymbol{d}_{h}^{n+1, k+1} \cdot \boldsymbol{v}_{h}^{\mathrm{s}} \\
=\frac{\gamma \mu}{h} \int_{\Sigma} \boldsymbol{u}_{h}^{n+1, k} \cdot \boldsymbol{v}_{h}^{\mathrm{s}}-\int_{\Sigma} \boldsymbol{\sigma}\left(\boldsymbol{u}_{h}^{n+1, k}, p_{h}^{n+1, k}\right) \boldsymbol{n} \cdot \boldsymbol{v}_{h}^{\mathrm{s}}
\end{aligned}
$$

for all $\left(\boldsymbol{v}_{h}^{\mathrm{s}}, \boldsymbol{r}_{h}\right) \in\left[\boldsymbol{V}_{h}^{\mathrm{s}}\right]^{2}$;

b) Fluid subproblem: find $\left(\boldsymbol{u}_{h}^{n+1, k+1}, p_{h}^{n+1, k+1}\right) \in \boldsymbol{V}_{h}^{\mathrm{f}} \times Q_{h}$, with $\boldsymbol{u}_{h}^{n+1, k+1}=\boldsymbol{u}_{\text {in }}\left(t_{n+1}\right)$ on $\Gamma^{\text {in }}$, such that

$$
\begin{gathered}
A_{\tau}^{\mathrm{f}}\left(\left(\boldsymbol{u}_{h}^{n+1, k+1}, p_{h}^{n+1, k+1}\right),\left(\boldsymbol{v}_{h}^{\mathrm{f}}, q_{h}\right)\right)-\int_{\Sigma} \boldsymbol{u}_{h}^{n+1, k+1} \cdot \boldsymbol{\sigma}\left(\boldsymbol{v}_{h}^{\mathrm{f}},-q_{h}\right) \boldsymbol{n} \\
\quad+\frac{\gamma \mu}{h} \int_{\Sigma} \boldsymbol{u}_{h}^{n+1, k+1} \cdot \boldsymbol{v}_{h}^{\mathrm{f}}+\frac{\gamma_{0} h}{\gamma \mu} \int_{\Sigma}\left(p_{h}^{n+1, k+1}-p_{h}^{n+1, k}\right) q_{h} \\
=\int_{\Sigma} \boldsymbol{\sigma}\left(\boldsymbol{u}_{h}^{n+1, k}, p_{h}^{n+1, k}\right) \boldsymbol{n} \cdot \boldsymbol{v}_{h}^{\mathrm{f}}-\int_{\Sigma} \partial_{\tau} \boldsymbol{d}_{h}^{n+1, k+1} \cdot \boldsymbol{\sigma}\left(\boldsymbol{v}_{h}^{\mathrm{f}},-q_{h}\right) \boldsymbol{n} \\
\quad+\frac{\gamma \mu}{h} \int_{\Sigma} \partial_{\tau} \boldsymbol{d}_{h}^{n+1, k+1} \cdot \boldsymbol{v}_{h}^{\mathrm{f}}-\int_{\Gamma_{\text {out }}} p_{\text {out }}\left(t_{n+1}\right) \boldsymbol{v}_{h}^{\mathrm{f}} \cdot \boldsymbol{n}
\end{gathered}
$$

for all $\left(\boldsymbol{v}_{h}^{\mathrm{f}}, q_{h}\right) \in \boldsymbol{V}_{h}^{\mathrm{f}} \times Q_{h}$;

3. Set $\boldsymbol{u}_{h}^{n+1} \stackrel{\text { def }}{=} \boldsymbol{u}_{h}^{n+1, K+1}, p_{h}^{n+1} \stackrel{\text { def }}{=} p_{h}^{n+1, K+1}, \boldsymbol{d}_{h}^{n+1} \stackrel{\text { def }}{=} \boldsymbol{d}_{h}^{n+1, K+1}$ and $\dot{\boldsymbol{d}}_{h}^{n+1} \stackrel{\text { def }}{=} \dot{\boldsymbol{d}}_{h}^{n+1, K+1}$;

4. Go to next time-step.

stabilization operator $S$. For the latter term we have

$$
r_{2}=\mathcal{O}\left((\gamma \mu)^{\frac{1}{2}} \frac{\tau}{h^{\frac{1}{2}}}+\gamma \mu^{\frac{1}{2}} \frac{\tau}{h}+\frac{\gamma_{0}^{\frac{1}{2}}}{(\gamma \mu)^{\frac{1}{2}}}(h \tau)^{\frac{1}{2}}\right) .
$$

For fixed $h$, the convergence order in time is imposed by the weak-consistency of the time penalty stabilization, which scales as $\mathcal{O}\left(\tau^{\frac{1}{2}}\right)$. The other two terms scale as $\mathcal{O}(\tau)$ but with a constant depends on $1 / h$. Therefore, when refining both in $\tau$ and in $h$, the stability condition (57) (i.e., $\tau=\mathcal{O}(h)$ ) is not enough to ensure convergence. We must take $\tau=\mathcal{O}\left(h^{2}\right)$ in order to keep $r_{2}=\mathcal{O}(h)$. Such a choice is optimal in the energy norm if piecewise affine approximations 
are used in space.

In practice, the method suffers from a deterioration of the accuracy, due to the weak consistency of the time penalty stabilization term, which rates as $\mathcal{O}\left(\tau^{\frac{1}{2}}\right)$ in a fixed mesh. As proposed in [22], accuracy can be improved by performing one correction iteration (see, e.g., [109]). The stabilized explicit coupling scheme with $K \geq 0$ corrections iterations is given in Algorithm 8. Note that, for $K=0$ (i.e., without corrections) Algorithm 8 reduces to the original stabilized explicit coupling scheme (Algorithm 7). Conversely, for $K \rightarrow \infty$ (i.e., iterating until convergence) we recover the implicit coupling scheme (51)-(52), $\left(\boldsymbol{u}_{h}^{\star}, p_{h}^{\star}\right)=\left(\boldsymbol{u}_{h}^{n+1}, p_{h}^{n+1}\right)$.

One of the main features of Algorithm 8 is that, after $K \geq 0$ corrections, estimate (59) is expected to hold with (see, e.g., [81])

$$
r_{2}=\mathcal{O}\left(\frac{\tau^{K+1}}{h^{\frac{K+1}{2}}}+\frac{\tau^{K+1}}{h^{K+1}}+(h \tau)^{\frac{K+1}{2}}\right) .
$$

Therefore, one correction iteration (i.e., $K=1$ ) is enough to retrieve first order time accuracy in a fixed mesh, since (60) yields $r_{2}=\mathcal{O}\left(\tau^{2} / h+\tau^{2} / h^{2}+h \tau\right)$. Note that, in this case, overall $r_{2}=\mathcal{O}(h)$ accuracy can be ensured under the (weakened) condition $\tau=\mathcal{O}\left(h^{\frac{3}{2}}\right)$.
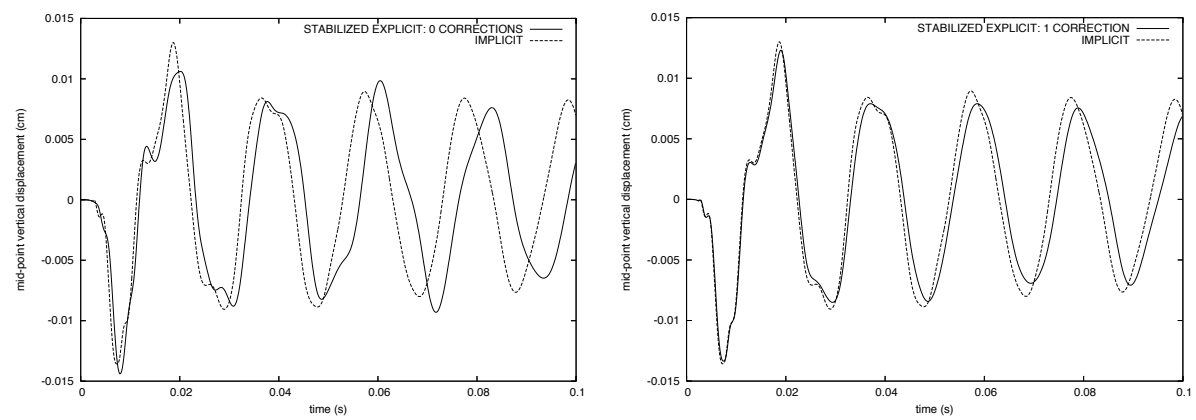

Figure 7: Interface mid-point $y$-displacement: stabilized explicit coupling (with and without correction) and implicit coupling (strongly enforced kinematic condition). From [22].

Remark 21 Algorithm 8 can be extended to the non-linear case (i.e., to problem (11)-(13)) without major difficulty. Roughly, the idea consists in replacing steps 2 and 3 of Algorithm 1 by the non-linear counterparts of the fluid and solid correction steps in Algorithm 8. We refer to [22, §5.5] for the details.

We conclude this subsection with a few numerical illustrations (from [22]) in the framework of the already mentioned straight vessel benchmark. Some snapshots of the fluid pressure and solid deformation (half a section) obtained with the non-linear version of Algorithm 8 are reported in Figure 6. The impact 
of the correction iterations is highlighted in Figure 7, where we compare the results with those obtained with a fully implicit coupling scheme (Algorithm 2). Figure 7 (left) shows that, without correction, the stabilized explicit scheme is unable to accurately represent the solution provided by the implicit coupling scheme. Figure 7 (right) shows that one correction iteration is sufficient to recover all the local features of the implicit coupling solution (i.e., we recover first order time accuracy).

\subsection{Robin-based semi-implicit coupling}

The theoretical and numerical results summarized in $\S 4$ show that the projection semi-implicit coupling scheme drastically improves the stability properties of conventional explicit coupling and the efficiency of implicit coupling. In spite of that, the scheme has two flaws. From a theoretical point of view, a non-convervative solid time discretization (i.e., with numerical dissipation) is required in the derivation of the energy stability estimate provided by Theorem 2 (see Remark 12). Secondly, though much less sensitive to the added-mass effect than explicit coupling, numerical evidence shows that the stability can be sensitive to changes in fluid-solid density ratio and other physical parameters (see the results reported in Figure 8 with the linear model (42)-(44)). As a matter of fact, the stability condition (47) provided by Theorem

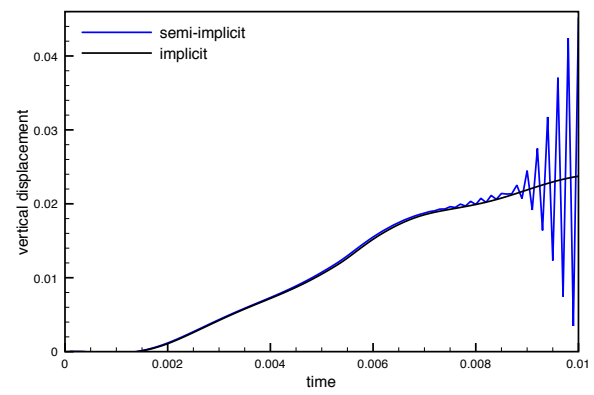

Figure 8: Comparison of the implicit and semi-implicit coupling: interface midpoint vertical displacement $\left(\rho^{\mathrm{f}}=1, \rho^{\mathrm{s}}=1.2 \times 10^{-2}, \mu=10\right)$. From [5].

2 depends on the solid and fluid densities. In this subsection we present an alternative semi-implicit coupling, proposed in [5], that circumvents these two shortcomings. The key idea consists in treating the explicit part of the coupling in a full weak sense, by using a specific Robin-Robin coupling derived from Remark 16 (see also Remark 19).

Let us consider the original semi-implicit coupling scheme (45)-(46) applied to linear coupled problem (42)-(44). As we have already seen, this coupling 
scheme is based on the following implicit-explicit time discretization of (44):

$$
\left.\begin{array}{r}
\boldsymbol{u}^{n+1} \cdot \boldsymbol{n}=\partial_{\tau} \boldsymbol{d}^{n+1} \cdot \boldsymbol{n} \\
\boldsymbol{\sigma}\left(\boldsymbol{d}^{n+\frac{1}{2}}\right) \boldsymbol{n}^{\mathrm{s}}=-\boldsymbol{\sigma}\left(\widetilde{\boldsymbol{u}}^{n}, p^{n+1}\right) \boldsymbol{n}
\end{array}\right\} \quad \text { on } \Sigma,
$$

Note that, in (46), the kinematic interface conditions $(61)_{1}$ and (62) are both strongly imposed (i.e., built in the finite element space). Instead, we now propose to treat the explicit part of the coupling (i.e., the viscous-structure coupling) weakly, by using the following explicit Robin-Robin treatment, derived from (54):

$$
\left.\begin{array}{c}
\boldsymbol{u}^{n+1} \cdot \boldsymbol{n}=\partial_{\tau} \boldsymbol{d}^{n+1} \cdot \boldsymbol{n} \\
\boldsymbol{\sigma}\left(\boldsymbol{d}^{n+\frac{1}{2}}\right) \boldsymbol{n}^{\mathrm{s}}+\frac{\gamma \mu}{h} \partial_{\tau} \boldsymbol{d}^{n+1}=\frac{\gamma \mu}{h} \widetilde{\boldsymbol{u}}^{n}-\boldsymbol{\sigma}\left(\widetilde{\boldsymbol{u}}^{n}, p^{n+1}\right) \boldsymbol{n}
\end{array}\right\} \quad \text { on } \quad \Sigma,
$$

Note that, in contrast to (54), the pressure-structure coupling remains implicit and the kinematic interface condition $(61)_{1}$ is strongly enforced (as in the original semi-implicit coupling scheme).

The proposed Robin based semi-implicit coupling scheme, applied to the linear coupled problem (42)-(44), reads therefore as follows (compare with (45)(46)): for $n \geq 0$,

- Implicit step (pressure-solid coupling): find $\left(\boldsymbol{u}_{h}^{n+1}, p_{h}^{n+1}, \boldsymbol{d}_{H}^{n+1}, \dot{\boldsymbol{d}}_{H}^{n+1}\right) \in$ $\boldsymbol{V}_{h}^{\mathrm{f}} \times Q_{h}^{\mathrm{f}} \times\left[\boldsymbol{V}_{H}^{\mathrm{s}}\right]^{2}$ such that

$$
\begin{aligned}
& \left\{\begin{array}{l}
\boldsymbol{u}_{h}^{n+1}=\boldsymbol{u}_{\text {in }}\left(t_{n+1}\right) \quad \text { on } \Gamma^{\text {in }} \\
\boldsymbol{u}_{h}^{n+1}=\Pi_{h}\left(\partial_{\tau} \boldsymbol{d}_{H}^{n+1}\right) \quad \text { on } \Sigma \\
\frac{\rho^{\mathrm{f}}}{\tau} \int_{\Omega^{\mathrm{f}}}\left(\boldsymbol{u}_{h}^{n+1}-\widetilde{\boldsymbol{u}}_{h}^{n}\right) \cdot \boldsymbol{v}_{h}^{\mathrm{f}}-\int_{\Omega^{\mathrm{f}}} p_{h}^{n+1} \operatorname{div} \boldsymbol{v}_{h}^{\mathrm{f}}+\int_{\Omega^{\mathrm{f}}} q_{h} \operatorname{div} \boldsymbol{u}_{h}^{n+1} \\
=-\int_{\Gamma_{\text {out }}} p_{\text {out }}\left(t_{n+1}\right) \boldsymbol{v}_{h}^{\mathrm{f}} \cdot \boldsymbol{n} \quad \forall\left(\boldsymbol{v}_{h}^{\mathrm{f}}, q_{h}\right) \in \boldsymbol{V}_{\Sigma \cup \Gamma^{\mathrm{in}}, h}^{\mathrm{f}} \times Q_{h}^{\mathrm{f}},
\end{array}\right. \\
& \left\{\begin{array}{l}
\frac{\rho^{\mathrm{s}}}{\tau} \int_{\Omega^{\mathrm{s}}} \partial_{\tau} \dot{\boldsymbol{d}}_{H}^{n+1} \cdot \boldsymbol{v}_{H}^{\mathrm{s}}+a^{\mathrm{s}}\left(\boldsymbol{d}_{H}^{n+\frac{1}{2}}, \boldsymbol{v}_{H}^{\mathrm{s}}\right)+\rho^{\mathrm{s}} \int_{\Omega^{\mathrm{s}}}\left(\dot{\boldsymbol{d}}_{H}^{n+\frac{1}{2}}-\partial_{\tau} \boldsymbol{d}_{H}^{n+1}\right) \cdot \boldsymbol{r}_{H} \\
+\frac{\gamma \mu}{h} \int_{\Sigma} \partial_{\tau} \boldsymbol{d}_{H}^{n+1} \cdot \boldsymbol{v}_{H}^{\mathrm{s}}=\frac{\gamma \mu}{h} \int_{\Sigma} \widetilde{\boldsymbol{u}}_{h}^{n} \cdot \boldsymbol{v}_{H}^{\mathrm{s}}-2 \mu \int_{\Sigma} \boldsymbol{\epsilon}\left(\widetilde{\boldsymbol{u}}_{h}^{n}\right) \boldsymbol{n} \cdot \boldsymbol{v}_{H}^{\mathrm{s}} \\
-\left\langle\mathcal{R}_{p}\left(\boldsymbol{u}_{h}^{n+1}, p_{h}^{n+1}\right), L_{h}\left(\left.\boldsymbol{v}_{H}^{\mathrm{s}}\right|_{\Sigma}\right)\right\rangle \quad \forall\left(\boldsymbol{v}_{H}^{\mathrm{s}}, \boldsymbol{r}_{H}\right) \in\left[\boldsymbol{V}_{H}^{\mathrm{s}}\right]^{2} ;
\end{array}\right.
\end{aligned}
$$


- Explicit step (viscous-solid coupling): find $\widetilde{\boldsymbol{u}}_{h}^{n+1} \in \boldsymbol{V}_{h}^{\mathrm{f}}$ such that

$$
\left\{\begin{array}{l}
\widetilde{\boldsymbol{u}}_{h}^{n+1}=\boldsymbol{u}_{\mathrm{in}}\left(t_{n+1}\right) \quad \text { on } \quad \Gamma^{\mathrm{in}} \\
\frac{\rho^{\mathrm{f}}}{\tau} \int_{\Omega^{\mathrm{f}}}\left(\widetilde{\boldsymbol{u}}_{h}^{n+1}-\boldsymbol{u}_{h}^{n+1}\right) \cdot \widetilde{\boldsymbol{v}}_{h}^{\mathrm{f}}+2 \mu \int_{\Omega^{\mathrm{f}}} \boldsymbol{\epsilon}\left(\widetilde{\boldsymbol{u}}_{h}^{n+1}\right): \boldsymbol{\epsilon}\left(\widetilde{\boldsymbol{v}}_{h}^{\mathrm{f}}\right)+\frac{\gamma \mu}{h} \int_{\Sigma} \widetilde{\boldsymbol{u}}_{h}^{n+1} \cdot \widetilde{\boldsymbol{v}}_{h}^{\mathrm{f}} \\
=\frac{\gamma \mu}{h} \int_{\Sigma} \partial_{\tau} \boldsymbol{d}_{H}^{n+1} \cdot \widetilde{\boldsymbol{v}}_{h}^{\mathrm{f}}+2 \mu \int_{\Sigma} \boldsymbol{\epsilon}\left(\widetilde{\boldsymbol{u}}_{h}^{n}\right) \boldsymbol{n} \cdot \widetilde{\boldsymbol{v}}_{h}^{\mathrm{f}} \quad \forall \widetilde{\boldsymbol{v}}_{h}^{\mathrm{f}} \in \boldsymbol{V}_{\Gamma^{\mathrm{in}}, h}^{\mathrm{f}} .
\end{array}\right.
$$

Note that in (63) we have considered a conservative time discretization for the structure.

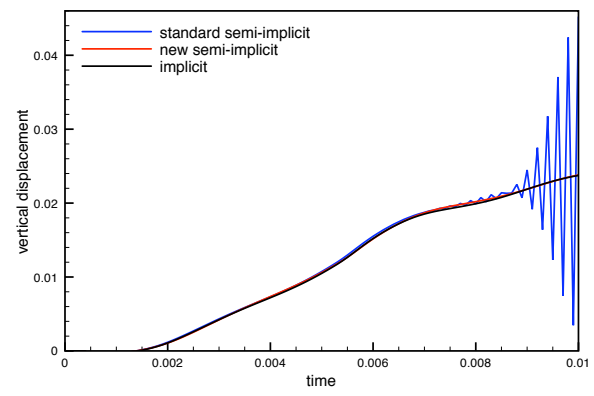

Figure 9: Comparison of the implicit, semi-implicit and Robin based semiimplicit coupling schemes: interface mid-point vertical displacement $\left(\rho^{\mathrm{f}}=1\right.$, $\left.\rho^{\mathrm{s}}=1.2 \times 10^{-2}, \mu=10\right)$. From [5].

Let us define the energy of the discrete coupled system, at time level $n$, as:

$$
E^{n}=\frac{\rho^{\mathrm{f}}}{2}\left\|\widetilde{\boldsymbol{u}}_{h}^{n}\right\|_{0, \Omega^{\mathrm{f}}}^{2}+\frac{\rho^{\mathrm{s}}}{2}\left\|\dot{\boldsymbol{d}}_{H}^{n}\right\|_{0, \Omega^{\mathrm{s}}}^{2}+\frac{1}{2} a^{\mathrm{s}}\left(\boldsymbol{d}_{H}^{n}, \boldsymbol{d}_{H}^{n}\right) .
$$

The following result (from [5]) states the energy based stability of the Robinbased semi-implicit coupling scheme (63)-(64).

Theorem 5 Let $\left(\widetilde{\boldsymbol{u}}_{h}^{n+1}, p_{h}^{n+1}, \boldsymbol{d}_{h}^{n+1}, \dot{\boldsymbol{d}}_{h}^{n+1}\right)$ be given by (63)-(64) and assume that the system is isolated (i.e., $p_{\text {out }}=0$ and $\boldsymbol{u}_{\mathrm{in}}=\mathbf{0}$ ). For $\gamma \geq 4 C_{\mathrm{TI}}$ and $n \geq 1$, there holds

$$
\begin{array}{r}
E^{n}+\mu \sum_{m=0}^{n-1} \tau\left\|\boldsymbol{\epsilon}\left(\widetilde{\boldsymbol{u}}_{h}^{m+1}\right)\right\|_{0, \Omega^{\mathrm{f}}}^{2}+\frac{\gamma \mu}{h} \sum_{m=0}^{n-1} \tau\left\|\widetilde{\boldsymbol{u}}_{h}^{m+1}-\partial_{\tau} \boldsymbol{d}_{H}^{m+1}\right\|_{0, \Sigma}^{2}+\frac{\gamma \mu \tau}{h}\left\|\widetilde{\boldsymbol{u}}_{h}^{n}\right\|_{0, \Sigma}^{2} \\
\lesssim E^{0}+\mu \tau\left\|\boldsymbol{\epsilon}\left(\widetilde{\boldsymbol{u}}_{h}^{0}\right)\right\|_{0, \Omega^{\mathrm{f}}}^{2}+\frac{\gamma \mu \tau}{h}\left\|\widetilde{\boldsymbol{u}}_{h}^{0}\right\|_{0, \Sigma}^{2} \quad(69)
\end{array}
$$

Therefore, the semi-implicit coupling scheme (63)-(64) is energy stable under the condition $\tau=\mathcal{O}(h)$. 
Algorithm 9 Robin based semi-implicit coupling algorithm ([5]).

1. Explicit step: update fluid domain (mesh)

$$
\left\{\begin{array}{l}
\boldsymbol{d}_{h}^{\mathrm{f}, n+1}=\operatorname{Ext}_{h}\left(\left.\boldsymbol{d}_{H}^{n}\right|_{\Sigma}\right), \quad \widehat{\boldsymbol{w}}_{h}^{n+1}=\partial_{\tau} \boldsymbol{d}_{h}^{\mathrm{f}, n+1} \quad \text { in } \quad \Omega^{\mathrm{f}}, \\
\Omega^{\mathrm{f}, n+1}=\left(\boldsymbol{I}_{\Omega^{\mathrm{f}}}+\boldsymbol{d}_{h}^{\mathrm{f}, n+1}\right)\left(\Omega^{\mathrm{f}}\right) .
\end{array}\right.
$$

2. Implicit step (pressure-structure coupling):

- Fluid projection sub-step: find $\left(\boldsymbol{u}_{h}^{n+1}, p_{h}^{n+1}\right) \in \boldsymbol{V}_{h}{ }^{\mathrm{f}} \times Q_{h}^{\mathrm{f}}$ such that

$$
\left\{\begin{array}{l}
\boldsymbol{u}_{h}^{n+1}=\boldsymbol{u}_{\text {in }}\left(t_{n+1}\right) \quad \text { on } \quad \Gamma^{\mathrm{in}}, \\
\widehat{\boldsymbol{u}}_{h}^{n+1}=\Pi_{h}\left(\partial_{\tau} \boldsymbol{d}_{H}^{n+1}\right) \quad \text { on } \quad \Sigma, \\
\frac{\rho^{\mathrm{f}}}{\tau} \int_{\Omega^{\mathrm{f}, n}}\left(\boldsymbol{u}_{h}^{n+1}-\widetilde{\boldsymbol{u}}_{h}^{n}\right) \cdot \boldsymbol{v}_{h}^{\mathrm{f}}-\int_{\Omega^{\mathrm{f}, n}} p_{h}^{n+1} \operatorname{div} \boldsymbol{v}_{h}^{\mathrm{f}}+\int_{\Omega^{\mathrm{f}, n}} q_{h} \operatorname{div} \boldsymbol{u}_{h}^{n+1} \\
\quad=-\int_{\Gamma_{\text {out }}} p_{\text {out }}\left(t_{n+1}\right) \boldsymbol{v}_{h}^{\mathrm{f}} \cdot \boldsymbol{n} \quad \forall\left(\widehat{\boldsymbol{v}}_{h}^{\mathrm{f}}, q_{h}\right) \in \boldsymbol{V}_{\Sigma \cup \Gamma^{\mathrm{in}}, h}^{\mathrm{f}} \times Q_{h}^{\mathrm{f}} ;
\end{array}\right.
$$

- Solid: find $\left(\boldsymbol{d}_{H}^{n+1}, \dot{\boldsymbol{d}}_{H}^{n+1}\right) \in\left[\boldsymbol{V}_{H}^{\mathrm{s}}\right]^{2}$ such that

$$
\left\{\begin{array}{r}
A_{\tau}^{\mathrm{s}}\left(\boldsymbol{d}_{H}^{n+1}, \dot{\boldsymbol{d}}_{H}^{n+1} ; \boldsymbol{v}_{H}^{\mathrm{s}}, \boldsymbol{r}_{H}\right)+\frac{\gamma \mu}{h} \int_{\Sigma^{n+1}} \partial_{\tau} \boldsymbol{d}_{H}^{n+1} \cdot \boldsymbol{v}_{H}^{\mathrm{s}} \\
=\frac{\gamma \mu}{h} \int_{\Sigma^{n+1}} \widetilde{\boldsymbol{u}}_{h}^{n} \cdot \boldsymbol{v}_{H}^{\mathrm{s}}-2 \mu \int_{\Sigma^{n+1}} \boldsymbol{\epsilon}\left(\widetilde{\boldsymbol{u}}_{h}^{n}\right) \boldsymbol{n} \cdot \boldsymbol{v}_{H}^{\mathrm{s}} \\
+\int_{\Sigma^{n+1}} p_{h}^{n+1} \boldsymbol{v}_{H}^{\mathrm{s}} \cdot \boldsymbol{n} \quad \forall \boldsymbol{v}_{H}^{\mathrm{s}}, \boldsymbol{r}_{H} \in\left[\boldsymbol{V}_{H}^{\mathrm{s}}\right]^{2}
\end{array}\right.
$$

3. Explicit step (viscous-structure coupling): find $\widehat{\widetilde{\boldsymbol{u}}}_{h}^{n+1} \in \boldsymbol{V}_{h}^{\mathrm{f}}$ such that

$$
\left\{\begin{aligned}
\widetilde{\boldsymbol{u}}_{h}^{n+1}=\boldsymbol{u}_{\text {in }}\left(t_{n+1}\right) \quad \text { on } \quad \Gamma^{\text {in }}, \\
\widetilde{A}_{\tau}^{\mathrm{f}}\left(\widetilde{\boldsymbol{u}}_{h}^{n+1}, \widetilde{\boldsymbol{v}}_{h}^{\mathrm{f}}\right)+\frac{\gamma \mu}{h} \int_{\Sigma^{n+1}} \widetilde{\boldsymbol{u}}_{h}^{n+1} \cdot \widetilde{\boldsymbol{v}}_{h}^{\mathrm{f}}=\frac{\gamma \mu}{h} \int_{\Sigma^{n+1}} \partial_{\tau} \boldsymbol{d}_{H}^{n+1} \cdot \widetilde{\boldsymbol{v}}_{h}^{\mathrm{f}} \\
\quad+2 \mu \int_{\Sigma^{n+1}} \boldsymbol{\epsilon}\left(\widetilde{\boldsymbol{u}}_{h}^{n}\right) \boldsymbol{n} \cdot \widetilde{\boldsymbol{v}}_{h}^{\mathrm{f}} \quad \forall \widetilde{\boldsymbol{v}}_{h}^{\mathrm{f}} \in \boldsymbol{V}_{\Gamma^{\mathrm{in}}, h}^{\mathrm{f}} ;
\end{aligned}\right.
$$

4. Go to next time step. 
Since the stability condition of Theorem 5 does not depend on the fluidsolid density ratio neither on the geometry of the domain, the semi-implicit coupling scheme (63)-(64) remains stable irrespectively of the added-mass effect. Moreover, thanks to the natural interface dissipation of the Robin-Robin coupling, a diffusive time marching in the structure is no longer needed to ensure stability. These observations are confirmed by the numerical results reported in Figure 9. The numerical instabilities shown in Figure 8, for the original semi-implicit coupling, are not present in the solution provided by the Robin based semi-implicit coupling scheme, which accurately predicts the results of the implicit coupling scheme.

Remark 22 Theorem 5 follows by a combination of the arguments involved in the proofs of Theorems 2 and 3. Let us notice that, here, we do not need to stabilize pressure fluctuations, that is, to introduce the weakly consistent artificial compressibility at the interface (55). Indeed, due to the implicit treatment (63) of the pressure-solid coupling, no artificial interface pressure perturbations appears in the energy estimate (69).

Remark 23 Theorem 5 can be extended to the case in which, instead of the pressure-Darcy formulation $(63)_{1}$, we consider the pressure-Poisson formulation of the projection step (see Remark 9). We refer to [5, §4.3] for the details.

The non-linear counterpart of the semi-implicit coupling scheme (63)-(64) (namely, the Robin based counterpart of Algorithm 6) is detailed in Algorithm 9. Here, Ext $_{h}$ stands for a discrete version of the lifting operator Ext, the solid mass and stiffness contribution are given by

$$
\begin{aligned}
A_{\tau}^{\mathrm{s}}\left(\boldsymbol{d}_{H}^{n+1}, \dot{\boldsymbol{d}}_{H}^{n+1} ; \boldsymbol{v}_{H}^{\mathrm{s}}, \boldsymbol{r}_{H}\right) \stackrel{\text { def }}{=} \rho^{\mathrm{s}} \int_{\Omega^{\mathrm{s}}} \partial_{\tau} \dot{\boldsymbol{d}}_{H}^{n+1} \cdot \boldsymbol{v}_{H}^{\mathrm{s}} \\
\quad+\frac{1}{2} \int_{\Omega^{\mathrm{s}}}\left(\boldsymbol{\Pi}\left(\boldsymbol{d}_{H}^{n+1}\right)+\boldsymbol{\Pi}\left(\boldsymbol{d}_{H}^{n}\right)\right): \boldsymbol{\nabla} \boldsymbol{v}_{H}^{\mathrm{s}}+\rho^{\mathrm{s}} \int_{\Omega^{\mathrm{s}}}\left(\dot{\boldsymbol{d}}_{H}^{n+\frac{1}{2}}-\partial_{\tau} \boldsymbol{d}_{H}^{n+1}\right) \cdot \boldsymbol{r}_{H},
\end{aligned}
$$

while, for the fluid

$$
\begin{aligned}
\widetilde{A}_{\tau}^{\mathrm{f}}\left(\widetilde{\boldsymbol{u}}_{h}^{n+1}, \widetilde{\boldsymbol{v}}_{h}^{\mathrm{f}}\right) \stackrel{\text { def }}{=} \frac{\rho^{\mathrm{f}}}{\tau} \int_{\Omega^{\mathrm{f}, n+1}} \widetilde{\boldsymbol{u}}_{h}^{n+1} \cdot \widetilde{\boldsymbol{v}}_{h}^{\mathrm{f}}-\frac{\rho^{\mathrm{f}}}{\tau} \int_{\Omega^{\mathrm{f}, n}} \boldsymbol{u}_{h}^{n+1} \cdot \widetilde{\boldsymbol{v}}_{h}^{\mathrm{f}} \\
\quad+\frac{\rho^{\mathrm{f}}}{2} \int_{\Omega^{\mathrm{f}, n+1}}\left(\operatorname{div} \widetilde{\boldsymbol{u}}_{h}^{n}\right) \widetilde{\boldsymbol{u}}_{h}^{n+1} \cdot \widetilde{\boldsymbol{v}}_{h}^{\mathrm{f}}-\rho^{\mathrm{f}} \int_{\Omega^{\mathrm{f}, n+1}}\left(\operatorname{div} \boldsymbol{w}_{h}^{n+1}\right) \widetilde{\boldsymbol{u}}_{h}^{n+1} \cdot \widetilde{\boldsymbol{v}}_{h}^{\mathrm{f}} \\
\quad+\rho^{\mathrm{f}} \int_{\Omega^{\mathrm{f}, n+1}}\left(\widetilde{\boldsymbol{u}}_{h}^{n}-\boldsymbol{w}_{h}^{n+1}\right) \cdot \nabla \widetilde{\boldsymbol{u}}_{h}^{n+1} \cdot \widetilde{\boldsymbol{v}}_{h}^{\mathrm{f}}+2 \mu \int_{\Omega^{\mathrm{f}, n+1}} \boldsymbol{\epsilon}\left(\widetilde{\boldsymbol{u}}_{h}^{n+1}\right): \boldsymbol{\epsilon}\left(\widetilde{\boldsymbol{v}}_{h}^{\mathrm{f}}\right) .
\end{aligned}
$$

We conclude this subsection with an illustration of the numerical results obtained (in [5]) with Algorithm 9 (pressure-Poisson version) and the physiological test case considered in $\S 4$. Figure 10 presents some snapshots of the wall deformation and the fluid velocity fields at two time instants. Figure 11 shows that, even in this complex case, both the original and the Robin based semi-implicit coupling schemes provide a prediction that compares well to the reference implicit solution. 

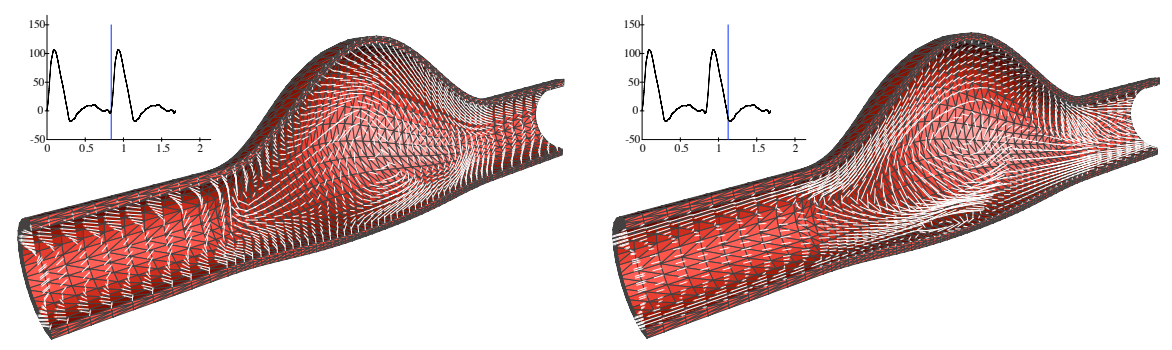

Figure 10: Robin based semi-implicit coupling: snapshots of the solid deformation and fluid velocity field at two different time instants. From [5].

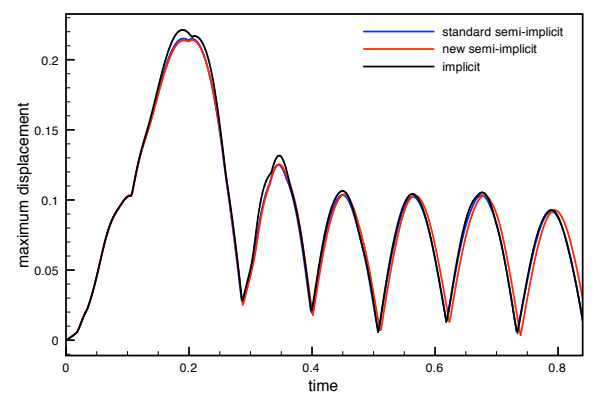

Figure 11: Comparison of the implicit, standard semi-implicit and Robin based semi-implicit coupling schemes: maximal displacement of the structure. From [5].

\section{Conclusion}

The work summarized in this review was devoted to the approximation and numerical resolution of the mechanical interaction between a viscous incompressible fluid and an elastic structure, with a strong added-mass effect. In this framework, standard explicit (or loosely coupled) schemes are known to be unstable, irrespectively of the discretization parameters. In the context of implicit coupling, we have seen that the exact evaluation of the cross-derivative of the Jacobian (shape terms) leads to robust Newton iterations. Yet, these procedures remain computationally expensive in real applications.

We have seen that implicit coupling can be avoided, without compromising stability, via the semi-implicit and the explicit coupling schemes described in $\S 4$ and $\S 5$, respectively. In particular, the explicit Robin-Robin treatment derived from the Nitsche treatment of the coupling yields added-mass effect free schemes. The price to pay is a perturbation of the truncation error, which enforces constraints on the rate of the discretization parameters (e.g., parabolic-CFL). Let us emphasize, that for standard loosely coupled schemes, 
these constraints do not cure the instabilities!

Many aspects of the studies presented in this review are open to further investigations (some of them are in progress). In the context of the numerical analysis of the schemes, the focus could be put on the convergence analysis in the linear case, for instance, in the spirit of $[84,7]$. Further investigations could also address the generalization of the stability analysis to the non-linear case (see, e.g., [83] for implicit coupling). Alternative explicit coupling schemes, motivated by the explicit Robin-Robin coupling (54), can be devised [20]:

(i) stabilized explicit coupling with a residual based treatment of the fluid stresses at the interface (instead of face-wise, as in the interface Nitsche's method);

(ii) unstabilized explicit Robin-Robin coupling without the consistency term $\int_{\Sigma}\left(\boldsymbol{u}_{h}^{n+1}-\partial_{\tau} \boldsymbol{d}_{h}^{n+1}\right) \cdot \boldsymbol{n} q_{h} ;$

(iii) explicit Robin-Robin coupling with (pressure-Poisson) projection scheme.

The variants (i) and (iii) can be proved to be energy stable. In particular, it is worth noticing that the combination of the Robin-Robin splitting with the pressure-Poisson treatment of the projection step, in (iii), leads to a natural stabilization of the time pressure fluctuations. Numerical evidence suggests that also (ii) is energy stable, but the analysis does not seem to be straightforward. The incremental displacement and displacement-velocity correction schemes recently introduced in $[44,50]$ will also be the topic of forthcoming investigations. An interesting feature of these loosely coupled procedures is that they can be interpreted as optimally consistent kinematic perturbations of an underlying implicit coupling scheme.

Regarding the applications, we plan to incorporate some of the proposed procedures in the context of the simulation of the fluid-structure interaction phenomena in the heart (see [118]), using the reduced valves models recently proposed in [4]. At last, in the context of inverse problems in blood flows (e.g., wall parameters estimation), some promising results [18], on the estimation of the mechanical properties of the vessel wall, have already been obtained by adapting the filtering techniques recently developed in [27].

\section{Acknowledgements}

The work summarized in this paper is the result of many fruitful collaborations. I deeply acknowledge the contributions of Matteo Astorino, Erik Burman, Franz Chouly, Jean-Frédéric Gerbeau, Céline Grandmont and Marwan Moubachir. I wish to thank also Vincent Martin, for his feedback on the first version of the manuscript (chapter 2 of my HDR dissertation). 


\section{References}

[1] G. Allaire. Conception optimale de structures, volume 58 of Mathématiques $\&$ Applications. Springer, 2007.

[2] D.N. Arnold. An interior penalty finite element method with discontinuous elements. SIAM J. Numer. Anal., 19(4):742-760, 1982.

[3] D.N. Arnold, F. Brezzi, B. Cockburn, and D. Marini. Discontinuous Galerkin methods for elliptic problems. In Discontinuous Galerkin methods (Newport, RI, 1999), volume 11 of Lect. Notes Comput. Sci. Eng., pages 89-101. Springer, 2000.

[4] M. Astorino. Interaction fluide-structure dans le système cardiovasculaire. Analyse numérique et simulation. PhD thesis, Université Paris VI, France, 2010.

[5] M. Astorino, F. Chouly, and M. A. Fernández. Robin based semi-implicit coupling in fluid-structure interaction: Stability analysis and numerics. SIAM J. Sci. Comput., 31(6):4041-4065, 2009.

[6] M. Astorino, J.-F. Gerbeau, O. Pantz, and K.-F. Traoré. Fluid-structure interaction and multi-body contact: application to aortic valves. Comput. Methods Appl. Mech. Engrg., 198(45-46):3603-3612, 2009.

[7] M. Astorino and C. Grandmont. Convergence analysis of a projection semi-implicit coupling scheme for fluid-structure interaction problems. Numer. Math., 116:721-767, 2010.

[8] S. Badia and R. Codina. On some fluid-structure iterative algorithms using pressure segregation methods. Application to aeroelasticity. Internat. J. Numer. Methods Engrg., 72(1):46-71, 2007.

[9] S. Badia, F. Nobile, and C. Vergara. Fluid-structure partitioned procedures based on Robin transmission conditions. J. Comp. Phys., 227:7027-7051, 2008.

[10] S. Badia, F. Nobile, and C. Vergara. Robin-Robin preconditioned Krylov methods for fluid-structure interaction problems. Comput. Methods Appl. Mech. Engrg., 198(33-36):2768-2784, 2009.

[11] S. Badia, A. Quaini, and A. Quarteroni. Modular vs. non-modular preconditioners for fluid-structure systems with large added-mass effect. Comput. Methods Appl. Mech. Engrg., 197(49-50):4216-4232, 2008.

[12] S. Badia, A. Quaini, and A. Quarteroni. Splitting methods based on algebraic factorization for fluid-structure interaction. SIAM J. Sci. Comput., 30(4):1778-1805, 2008.

[13] G.A. Baker. Finite element methods for elliptic equations using nonconforming elements. Math. Comp., 31(137):45-59, 1977. 
[14] K.J. Bathe and H. Zhang. Finite element developments for general fluid flows with structural interactions. Int. J. Num. Meth. Engng., 2004.

[15] Y. Bazilevs, V.M. Calo, T.J.R. Hughes, and Y. Zhang. Isogeometric fluidstructure interaction: theory, algorithms, and computations. Comput. Mech., 43(1):3-37, 2008.

[16] R. Becker, P. Hansbo, and R. Stenberg. A finite element method for domain decomposition with non-matching grids. M2AN Math. Model. Numer. Anal., 37(2):209-225, 2003.

[17] C. Bernardi, Y. Maday, and A. T. Patera. Domain decomposition by the mortar element method. In Asymptotic and numerical methods for partial differential equations with critical parameters (Beaune, 1992), volume 384 of NATO Adv. Sci. Inst. Ser. C Math. Phys. Sci., pages 269-286. Kluwer Acad. Publ., Dordrecht, 1993.

[18] C. Bertoglio, D. Chapelle, M.A. Fernández, J.-F. Gerbeau, and P. Moireau. Filtering-based data assimilation in vascular fluid-structure interaction through displacement measurements at the interface. In $I V$ European Congress on Computational Mechanics (ECCM IV): Solids, Structures and Coupled Problems in Engineering, 2010.

[19] P. N. Brown and Y. Saad. Convergence theory of nonlinear Newton-Krylov algorithms. SIAM J. Optim., 4(2):297-330, 1994.

[20] E. Burman and M.A. Fernández. Robin-based explicit coupling schemes in incompressible fluid-structure interaction. In preparation.

[21] E. Burman and M.A. Fernández. Stabilized explicit coupling for fluidstructure interaction using Nitsche's method. C. R. Math. Acad. Sci. Paris, 345(8):467-472, 2007.

[22] E. Burman and M.A. Fernández. Stabilization of explicit coupling in fluidstructure interaction involving fluid incompressibility. Comput. Methods Appl. Mech. Engrg., 198(5-8):766-784, 2009.

[23] E. Burman and P. Hansbo. A unified stabilized method for Stokes' and Darcy's equations. J. Comput. Appl. Math., 198:35-51, 2007.

[24] E. Burman and P. Zunino. A domain decomposition method based on interior penalties for advection-diffusion-reaction problems. Siam Jour. Num. Anal., 44:1612-1638, 2006.

[25] P. Causin, J.-F. Gerbeau, and F. Nobile. Added-mass effect in the design of partitioned algorithms for fluid-structure problems. Comput. Methods Appl. Mech. Engrg., 194(42-44):4506-4527, 2005.

[26] D. Chapelle and K.J. Bathe. The Finite Element Analysis of Shells Fundamentals. Springer, 2003. 
[27] D. Chapelle, P. Moireau, and P. Le Tallec. Robust filtering for joint stateparameter estimation in distributed mechanical systems. Discrete Contin. Dyn. Syst., 23(1-2):65-84, 2009.

[28] Y. Cheng, H. Oertel, and T. Schenkel. Fluid-structure coupled CFD simulation of the left ventricular flow during filling phase. Ann. Biomed. Eng., 33(5):567-576, 2005.

[29] A.J. Chorin. On the convergence of discrete approximations to the NavierStokes equations. Math. Comp., 23:341-353, 1969.

[30] P.G. Ciarlet. Mathematical elasticity. Vol. I, volume 20 of Studies in Mathematics and its Applications. North-Holland, 1988.

[31] J. Degroote, S. Annerel, and J. Vierendeels. Stability analysis of GaussSeidel iterations in a partitioned simulation of fluid-structure interaction. Comp. \&3 Struct., 88(5-6):263-271, 2010.

[32] J. Degroote, K.-J. Bathe, and J. Vierendeels. Performance of a new partitioned procedure versus a monolithic procedure in fluid-structure interaction. Comp. \&5 Struct., 87(11-12):793-801, 2009.

[33] J. Degroote, A. Swillens, P. Bruggeman, R. Haelterman, P. Segers, and J. Vierendeels. Simulation of fluid-structure interaction with the interface artificial compressibility method. Int. J. Numer. Meth. Biomed. Engng., 26(3-4):276-289, 2010.

[34] P. Degroote, J. Bruggeman, R. Haelterman, and J. Vierendeels. Stability of a coupling technique for partitioned solvers in FSI applications. Comp. E3 Struct., 86(23-24):2224-2234, 2008.

[35] S. Deparis. Numerical Analysis of Axisymmetric Flows and Methods for Fluid-Structure Interaction Arising in Blood Flow Simulation. PhD thesis, EPFL, Switzerland, 2004.

[36] S. Deparis, M. Discacciati, G. Fourestey, and A. Quarteroni. Fluidstructure algorithms based on Steklov-Poincaré operators. Comput. Methods Appl. Mech. Engrg., 195(41-43):5797-5812, 2006.

[37] S. Deparis, M.A. Fernández, and L. Formaggia. Acceleration of a fixed point algorithm for fluid-structure interaction using transpiration conditions. M2AN Math. Model. Numer. Anal., 37(4):601-616, 2003.

[38] W. Dettmer and D. Perić. A computational framework for fluid-rigid body interaction: finite element formulation and applications. Comput. Methods Appl. Mech. Engrg., 195(13-16):1633-1666, 2006.

[39] N. Diniz dos Santos, J.-F. Gerbeau, and J.-F. Bourgat. A partitioned fluid-structure algorithm for elastic thin valves with contact. Comput. Methods Appl. Mech. Engrg., 197(19-20):1750-1761, 2008. 
[40] J. Donéa, S. Giuliani, and J. P. Halleux. An arbitrary Lagrangian-Eulerian finite element method for transient dynamic fluid-structure interactions. Comp. Meth. Appl. Mech. Engng., pages 689-723, 1982.

[41] C. Farhat, M. Lesoinne, and P. Le Tallec. Load and motion transfer algorithms for fluid/structure interaction problems with non-matching discrete interfaces: Momentum and energy conservation, optimal discretization and application to aeroelasticity. Comput. Methods Appl. Mech. Engrg., 157:95-114, 1998.

[42] C. Farhat, K. van der Zee, and Ph. Geuzaine. Provably second-order time-accurate loosely-coupled solution algorithms for transient nonlinear aeroelasticity. Comput. Methods Appl. Mech. Engrg., 195(17-18):19732001, 2006.

[43] C.A. Felippa, K.C. Park, and C. Farhat. Partitioned analysis of coupled mechanical systems. Comput. Methods Appl. Mech. Engrg., 190(2425):3247-3270, 2001.

[44] M.A. Fernández. Incremental displacement-correction schemes for the explicit coupling of a thin structure with an incompressible fluid. C. R. Acad. Sci. Paris Sér. I Math., 2011. In press. DOI: $10.1016 /$ j.crma.2011.03.001.

[45] M.A. Fernández, L. Formaggia, J.-F. Gerbeau, and A. Quarteroni. The derivation of the equations for fluids and structures. In Cardiovascular mathematics, volume 1 of MS\&A. Model. Simul. Appl., pages 77-121. Springer, 2009.

[46] M.A. Fernández and J.-F. Gerbeau. Algorithms for fluid-structure interaction problems. In Cardiovascular mathematics, volume 1 of $M S E S A$. Model. Simul. Appl., pages 307-346. Springer, 2009.

[47] M.A. Fernández, J.-F. Gerbeau, and C. Grandmont. A projection algorithm for fluid-structure interaction problems with strong added-mass effect. C. R. Math. Acad. Sci. Paris, 342(4):279-284, 2006.

[48] M.A. Fernández, J.F. Gerbeau, and C. Grandmont. A projection semi-implicit scheme for the coupling of an elastic structure with an incompressible fluid. Int. J. Num. Meth. Engrg., 69(4):794-821, 2007.

[49] M.A. Fernández and M. Moubachir. A Newton method using exact Jacobians for solving fluid-structure coupling. Comp. ES Struct., 83:127$142,2005$.

[50] M.A. Fernández and J. Mullaert. Displacement-velocity correction schemes for incompressible fluid-structure interaction. Submitted to C. R. Acad. Sci. Paris Sér. I Math., http://hal.inria.fr/inria-00583126/en/. 
[51] C.A. Figueroa, I.E. Vignon-Clementel, K.E. Jansen, T.J.R. Hughes, and C.A. Taylor. A coupled momentum method for modeling blood flow in three-dimensional deformable arteries. Comput. Methods Appl. Mech. Engrg., 195(41-43):5685-5706, 2006.

[52] L. Formaggia, J.-F. Gerbeau, F. Nobile, and A. Quarteroni. On the coupling of 3D and 1D Navier-Stokes equations for flow problems in compliant vessels. Comp. Meth. Appl. Mech. Engrg., 191(6-7):561-582, 2001.

[53] L. Formaggia, K. Perktold, and A. Quarteroni. Basic mathematical models and motivations. In Cardiovascular mathematics, volume 1 of MSEA. Model. Simul. Appl., pages 47-75. Springer, 2009.

[54] C. Förster, W.A. Wall, and E. Ramm. Artificial added mass instabilities in sequential staggered coupling of nonlinear structures and incompressible viscous flows. Comput. Methods Appl. Mech. Engrg., 196(7):1278-1293, 2007.

[55] G. Fourestey and S. Piperno. A second-order time-accurate ALE Lagrange-Galerkin method applied to wind engineering and control of bridge profiles. Comput. Methods Appl. Mech. Engrg., 193(39-41):41174137, 2004.

[56] Y.C. Fung. Biomechanics, Mechanical properties of living tissues. Springer, 1993.

[57] M.J. Gander and L. Halpern. Optimized Schwarz waveform relaxation methods for advection reaction diffusion problems. SIAM J. Numer. Anal., 45(2):666-697, 2007.

[58] M.W. Gee, U. Küttler, and W. Wall. Truly monolithic algebraic multigrid for fluid-structure interaction. Int. J. Numer. Meth. Engng., 2010. To appear.

[59] J.-F. Gerbeau and M. Vidrascu. A quasi-Newton algorithm based on a reduced model for fluid-structure interactions problems in blood flows. Math. Model. Num. Anal., 37(4):631-648, 2003.

[60] J.-F. Gerbeau, M. Vidrascu, and P. Frey. Fluid-structure interaction in blood flows on geometries based on medical imaging. Comp. E3 Struct., 83(2-3):155-165, 2005.

[61] C. Grandmont and Y. Maday. Nonconforming grids for the simulation of fluid-structure interaction. In Domain decomposition methods, 10 (Boulder, CO, 1997), volume 218 of Contemp. Math., pages 262-270. Amer. Math. Soc., Providence, RI, 1998.

[62] J.-L. Guermond. Some implementation of projection methods for NavierStokes equations. M2AN Math. Model. Numer. Anal., 30:637-667, 1996. 
[63] J. L. Guermond, P. Minev, and J. Shen. An overview of projection methods for incompressible flows. Comput. Methods Appl. Mech. Engrg., 195(44-47):6011-6045, 2006.

[64] G. Guidoboni, R. Glowinski, N. Cavallini, and S. Canic. Stable looselycoupled-type algorithm for fluid-structure interaction in blood flow. $J$. Comp. Phys., 228(18):6916-6937, 2009.

[65] G. Guidoboni, R. Glowinski, N. Cavallini, S. Canic, and S. Lapin. A kinematically coupled time-splitting scheme for fluid-structure interaction in blood flow. Appl. Math. Lett., 22(5):684-688, 2009.

[66] M.E. Gurtin. An introduction to continuum mechanics, volume 158 of Mathematics in Science and Engineering. Academic Press Inc., 1981.

[67] P. Hansbo. Nitsche's method for interface problems in computational mechanics. GAMM-Mitt., 28(2):183-206, 2005.

[68] P. Hansbo and J. Hermansson. Nitsche's method for coupling nonmatching meshes in fluid-structure vibration problems. Computational Mechanics, 32(1-2):134-139, 2003.

[69] P. Hansbo, J. Hermansson, and T. Svedberg. Nitsche's method combined with space-time finite elements for ALE fluid-structure interaction problems. Comput. Methods Appl. Mech. Engrg., 193:4195-4206, 2004.

[70] M. Heil. An efficient solver for the fully coupled solution of largedisplacement fluid-structure interaction problems. Comput. Methods Appl. Mech. Engrg., 193(1-2):1-23, 2004.

[71] M. Heil, A.L. Hazel, and J. Boyle. Solvers for large-displacement fluidstructure interaction problems: Segregated vs. monolithic approaches. Comp. Mech., 43:91-101, 2008.

[72] B. Hübner, E. Walhorn, and D. Dinkle. A monolithic approach to fluidstructure interaction using space-time finite elements. Comp. Meth. Appl. Mech. Engng., 193:2087-2104, 2004.

[73] E. Järvinen, P. Räback, M. Lyly, and J.-P. Salenius. A method for partitioned fluid-structure interaction computation of flow in arteries. Medical Engineering \& Physics, 30(7):917-923, 2008.

[74] M.M. Joosten, W.G. Dettmer, and D. Perić. Analysis of the block Gauss-Seidel solution procedure for a strongly coupled model problem with reference to fluid-structure interaction. Internat. J. Numer. Methods Engrg., 78(7):757-778, 2009.

[75] M. Juntunen and R. Stenberg. Nitsche's method for general boundary conditions. Math. Comp., 78(267):1353-1374, 2009. 
[76] S.A. Kock, J.V. Nygaard, N. Eldrup, E.-T. Fründ, A. Klaerke, W.P. Paaske, E. Falk, and W. Yong Kim. Mechanical stresses in carotid plaques using mri-based fluid-structure interaction models. J. Biomech., 41(8):1651-1658, 2008.

[77] U. Küttler, M.W. Gee, C. Förster, A. Comerford, and W.A. Wall. Coupling strategies for biomedical fluid-structure interaction problems. Int. J. Numer. Meth. Biomed. Engng., 26(3-4):305-321, 2009.

[78] U. Küttler and W.A. Wall. Fixed-point fluid-structure interaction solvers with dynamic relaxation. Comp. Mech., 43(1):61-72, 2008.

[79] U. Küttler and W.A. Wall. Vector extrapolation for strong coupling fluidstructure interaction solvers. J. App. Mech., 76(2):021205, 2009.

[80] L. Lanoye, J. Vierendeels, P. Segers, and P. Verdonck. Vascular fluidstructure-interaction using Fluent and Abaqus software. J. Biomech., 39(Supplement 1):S440-S440, 2006.

[81] W. Layton, H. K. Lee, and J. Peterson. A defect-correction method for the incompressible Navier-Stokes equations. Appl. Math. Comput., 129(1):119, 2002.

[82] P. Le Tallec. Numerical methods for nonlinear three-dimensional elasticity. In Handbook of numerical analysis, Vol. III, pages 465-622. North-Holland, 1994.

[83] P. Le Tallec and P. Hauret. Energy conservation in fluid structure interactions. In Numerical methods for scientific computing. Variational problems and applications, pages 94-107. Internat. Center Numer. Methods Eng. (CIMNE), Barcelona, 2003.

[84] P. Le Tallec and S. Mani. Numerical analysis of a linearised fluid-structure interaction problem. Numer. Math., 87(2):317-354, 2000.

[85] P. Le Tallec and J. Mouro. Fluid structure interaction with large structural displacements. Comput. Meth. Appl. Mech. Engrg., 190:30393067, 2001.

[86] M. Lesoinne and C. Farhat. Higher-order subiteration-free staggered algorithm for nonlinear transient aeroelastic problems. AIAA Journal, 36(9):1754-1757, 1998.

[87] Z. Li and C. Kleinstreuer. Computational analysis of type II endoleaks in a stented abdominal aortic aneurysm model. J. Biomech., 39(14):2573$2582,2006$.

[88] Y. Maday. Analysis of coupled models for fluid-structure interaction of internal flows. In Cardiovascular mathematics, volume 1 of MSËA. Model. Simul. Appl., pages 279-306. Springer, 2009. 
[89] H.G. Matthies and J. Steindorf. Partitioned but strongly coupled iteration schemes for nonlinear fluid-structure interaction. Comp. \& Struct., 80(2730):1991-1999, 2002.

[90] D.P. Mok and W.A. Wall. Partitioned analysis schemes for the transient interaction of incompressible flows and nonlinear flexible structures. In W.A. Wall, K.U. Bletzinger, and K. Schweizerhof, editors, Trends in computational structural mechanics, Barcelona, 2001. CIMNE.

[91] D.P. Mok, W.A. Wall, and E. Ramm. Partitioned analysis approach for the transient, coupled response of viscous fluids and flexible structures. In W. Wunderlich, editor, Proceedings of the European Conference on Computational Mechanics. ECCM'99, TU Munich, 1999.

[92] D.P. Mok, W.A. Wall, and E. Ramm. Accelerated iterative substructuring schemes for instationary fluid-structure interaction. In Bathe. K.J., editor, Computational Fluid and Solid Mechanics, pages 1325-1328. Elsevier, 2001.

[93] H. Morand and R. Ohayon. Fluid-Structure Interaction: Applied Numerical Methods. John Wiley \& Sons, 1995.

[94] J. Mouro. Interactions fluide structure en grands déplacements. Résolution numérique et application aux composants hydrauliques automobiles. PhD thesis, Ecole Polytechnique, France, 1996.

[95] J. Nitsche. Über ein Variationsprinzip zur Lösung von DirichletProblemen bei Verwendung von Teilräumen, die keinen Randbedingungen unterworfen sind. Abh. Math. Sem. Univ. Hamburg, 36:9-15, 1971.

[96] F. Nobile. Numerical approximation of fluid-structure interaction problems with application to haemodynamics. PhD thesis, EPFL, Switzerland, 2001.

[97] F. Nobile and C. Vergara. An effective fluid-structure interaction formulation for vascular dynamics by generalized Robin conditions. SIAM J. Sci. Comput., 30(2):731-763, 2008.

[98] T. Nomura and T.J.R. Hughes. An arbitray Lagrangian-Eulerian finite element method for interaction of fluid and rigid body. Comput. Methods Appl. Mech. Eng., 95(1):115-138, 1992.

[99] S. Piperno. Simulation numérique de phénomènes d'interaction fluidestructure. PhD thesis, Ecole Nationale des Ponts et Chaussées, France, 1995.

[100] S. Piperno. Explicit/implicit fluid/structure staggered procedures with a structural predictor and fluid subcycling for 2D inviscid aeroelastic simulations. Internat. J. Numer. Methods Fluids, 25(10):1207-1226, 1997. 
[101] S. Piperno and P.E. Bournet. Numerical simulations of wind effects on flexible civil engineering structures. Rev. Eur. Élém. Finis, 8(5-6):659$687,2001$.

[102] S. Piperno and C. Farhat. Design of efficient partitioned procedures for the transient solution of aeroelastic problems. In Fluid-structure interaction, Innov. Tech. Ser., pages 23-49. Kogan Page Sci., 2003.

[103] A. Quaini and A. Quarteroni. A semi-implicit approach for fluid-structure interaction based on an algebraic fractional step method. Math. Models Methods Appl. Sci., 17(6):957-983, 2007.

[104] K. Riemslagh, J. Vierendeels, and E. Dick. An efficient coupling procedure for flexible wall fluid-structure interaction. In ECCOMAS 2000, Barcelona, 2000.

[105] S. Rugonyi and K.J. Bathe. On finite element analysis of fluid flows coupled with structural interaction. CMES - Comp. Modeling Eng. Sci., $2(2): 195-212,2001$.

[106] A.-V. Salsac, S.R. Sparks, J.M. Chomaz, and J.C. Lasheras. Evolution of the wall shear stresses during the progressive enlargement of symmetric abdominal aortic aneurysms. J. Fluid Mech., 550:19-51, 2006.

[107] M. Schäfer, M. Heck, and S. Yigit. An implicit partitioned method for the numerical simulation of fluid-structure interaction. In Fluid-structure interaction, volume 53 of Lect. Notes Comput. Sci. Eng., pages 171-194. Springer, 2006.

[108] J. Sokolowski and J.-P. Zolésio. Introduction to shape optimization, volume 16 of Springer Series in Computational Mathematics. Springer, 1992.

[109] H.J. Stetter. The defect correction principle and discretization methods. Numer. Math., 29:425-443, 1978.

[110] E. W. Swim and P. Seshaiyer. A nonconforming finite element method for fluid-structure interaction problems. Comput. Methods Appl. Mech. Engrg., 195(17-18):2088-2099, 2006.

[111] S. Sy and C.M. Murea. A stable time advancing scheme for solving fluidstructure interaction problem at small structural displacements. Comput. Methods Appl. Mech. Engrg., 198(2):210-222, 2008.

[112] R. Temam. Une méthode d'approximation de la solution des équations de Navier-Stokes. Bull. Soc. Math. France, 96:115-152, 1968.

[113] T.E. Tezduyar. Finite element methods for fluid dynamics with moving boundaries and interfaces. Arch. Comput. Methods Engrg., 8:83-130, 2001. 
[114] T.E. Tezduyar and S. Sathe. Modelling of fluid-structure interactions with the space-time finite elements: solution techniques. Internat. J. Numer. Methods Fluids, 54(6-8):855-900, 2007.

[115] M. Thiriet. Biology and Mechanics of Blood Flows. Part II: Mechanics and Medical Aspects. CRM Series in Mathematical Physics. Springer, 2008.

[116] J. Vierendeels, Lanoye. L., J. Degroote, and P. Verdonck. Implicit coupling of partitioned fluid-structure interaction problems with reduced order models. Comp. \& Struct., 85(11-14):970-976, 2007.

[117] W.A. Wall, S. Genkinger, and E. Ramm. A strong coupling partitioned approach for fluid-structure interaction with free surfaces. Comput. \& Fluids, 36(1):169-183, 2007.

[118] H. Watanabe, S. Sugiura, H. Kafuku, and T. Hisada. Multiphysics simulation of left ventricular filling dynamics using fluid-structure interaction finite element method. Biophysical Journal, 87(3):2074-2085, 2004.

[119] M.F. Wheeler. An elliptic collocation-finite element method with interior penalties. SIAM J. Numer. Anal., 15(1):152-161, 1978.

[120] L. Wiechert, T. Rabczuk, A. Comerford, R. Metzke, and W.A. Wall. Towards stresses and strains in the respiratory system. In Mathematical and numerical modelling of the human lung, volume 23 of ESAIM Proc., pages 98-113. EDP Sci., 2008.

[121] X.L. Yang, Y. Liu, and J.M. Yang. Fluid-structure interaction in a pulmonary arterial bifurcation. J. Biomech., 40(12):2694-2699, 2007. 